\title{
Electrical manipulation of liquids at interfaces
}

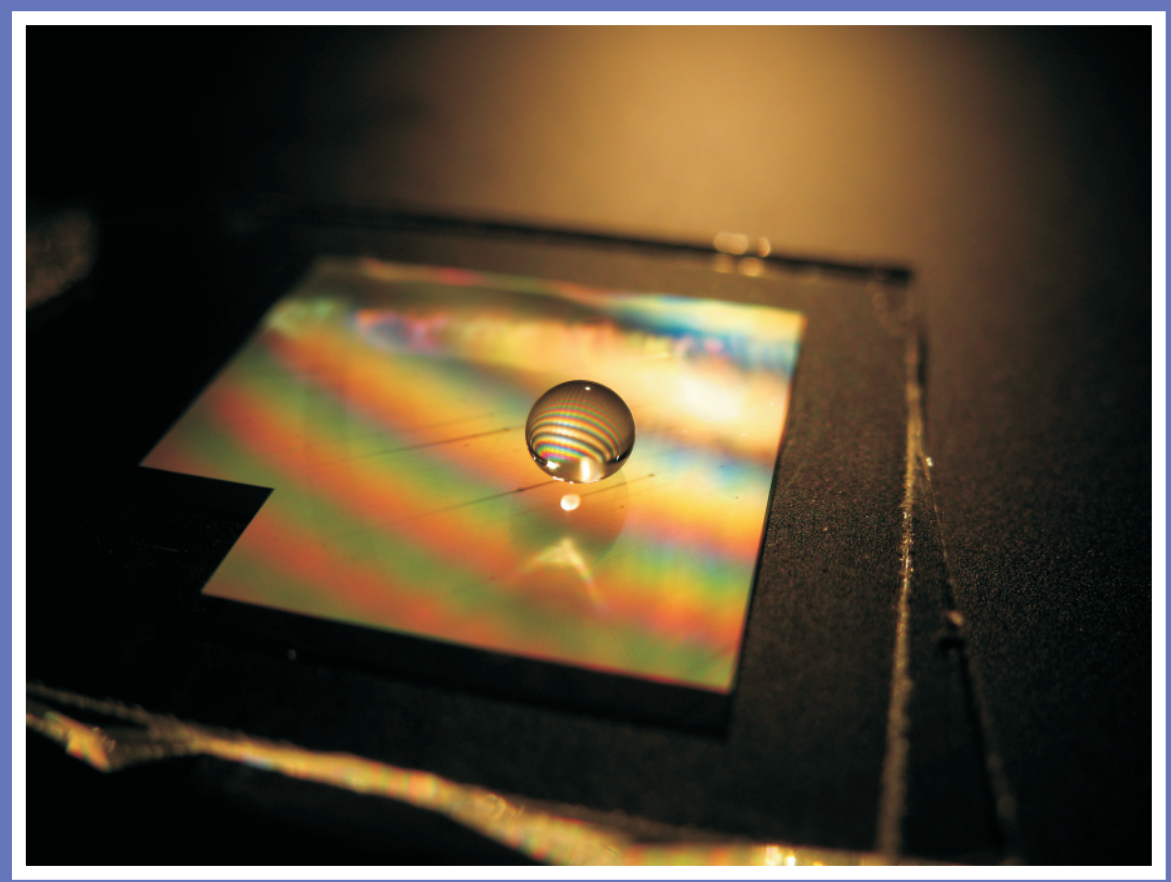

Gor Manukyan 
Electrical manipulation of liquids at interfaces 



\title{
Electrical manipulation of liquids
}

\author{
at interfaces
}

\section{PROEFSCHRIFT}

ter verkrijging van de graad van doctor aan de Universiteit Twente, op gezag van de rector magnificus, prof.dr.H. Brinksma,

volgens besluit van het College van Promoties

in het openbaar te verdedigen

op 16 September 2011

door

Gor Manukyan

geboren op 24 april 1983

Martuni, Armenia 

Dit proefschrift is goedgekeurd door de promotores

prof. dr. F. Mugele

en

prof. dr. ir. R.G.H. Lammertink 



\section{Contents}

1 Introduction 1

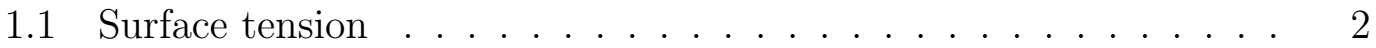

1.2 Superhydrophobic surfaces . . . . . . . . . . . . . 4

1.2.1 Cassie-Baxter state . . . . . . . . . . . . . . 7

1.2.2 Wenzel state. . . . . . . . . . . . . . . . 8

1.3 Transitions between Cassie-Baxter and Wenzel states . . . . . . . 9

1.4 Liquids in an electric field . . . . . . . . . . . . . . . 10

1.4.1 Electrowetting . . . . . . . . . . . . . . 11

1.4.2 Electrodiffusion . . . . . . . . . . . . . . . 13

1.5 Outline of the thesis . . . . . . . . . . . . . 17

2 Electrical switching of wetting states on superhydrophobic surfaces $\quad 19$

2.1 Abstract . . . . . . . . . . . . . . . . . . 19

2.2 Introduction . . . . . . . . . . . . . . . . . 20

2.3 Materials and methods . . . . . . . . . . . . . . . . 21

2.3.1 Sample preparation . . . . . . . . . . . . 21

2.3.2 Experimental setup . . . . . . . . . . . . . . 21

2.4 Observations . . . . . . . . . . . . . . . . . . . 22

2.5 The model . . . . . . . . . . . . . . . . . . 26 26

2.6 Results and disscussion . . . . . . . . . . . . . . . . 28

2.7 Conclusions . . . . . . . . . . . . . . . . . 31 
3 Electrically induced reversible transitions on superhydrophobic surfaces 33

3.1 Abstract . . . . . . . . . . . . . . . . . 33

3.2 Introduction . . . . . . . . . . . . . . . . . 34

3.3 Wenzel to Cassie-Baxter transitions using low frequency oscillations 35

3.3.1 Materials and methods ............ . 35

3.3 .2 Observations . . . . . . . . . . . . . . . 36

3.4 Reversible transitions using patterned electrods . . . . . . . . 37

3.4 .1 Materials and methods . . . . . . . . . . . 38

3.4 .2 Observations . . . . . . . . . . . . . . 38

3.5 Conclusions . . . . . . . . . . . . . . . . . . . . 41

4 Electric field driven instabilities on superhydrophobic surfaces 43

4.1 abstract . . . . . . . . . . . . . . . . . . . . . . 43

4.2 Introduction . . . . . . . . . . . . . . . 44

4.3 The model . . . . . . . . . . . . . . . . . . . 45

4.4 Materials and methods . . . . . . . . . . . . . . . . . 46

4.5 Results and discussion . . . . . . . . . . . . . . . . . 47

4.6 Conclusion . . . . . . . . . . . . . . . . . 52

5 (Electro)-wetting of a drop on a sphere 55

5.1 Introduction . . . . . . . . . . . . . . . . . 56 56

5.2 Materials and methods . . . . . . . . . . . 59

5.2 .1 Electrowetting setup . . . . . . . . . . . . 59

5.2 .2 Substrate preparation . . . . . . . . . . . . . 59

5.2 .3 Image processing . . . . . . . . . . . . . . . 60

5.2.3.1 Contact angle measurements . . . . . . . 60

5.2.3.2 Effective interfacial energy calculations from experimental data . . . . . . . . . . . . 60

5.2.4 Analytical effective interfacial energy calculations . . . . . 61

5.3 Results and disscusion . . . . . . . . . . . . . . 63

5.4 Conclusions . . . . . . . . . . . . . . . . . . . . . 69 
6 Direct observation of non equilibrium electroosmotic instability 71 6.1 Abstract ....................... . . 71

6.2 Introduction . . . . . . . . . . . . . . . . . 72

6.3 Materials and methods . . . . . . . . . . . . . . 73

6.4 Results and discussion . . . . . . . . . . . . . 74

6.5 Conclusions ...................... . . 79

7 Summary and outlook 81

7.1 Summary ........................ 81

7.2 Outlook ........................ 84

References

$\begin{array}{ll}\text { Samenvatting } & 103\end{array}$

$\begin{array}{ll}\text { Acknowledgements } & 107\end{array}$

About the author $\quad 110$ 


\section{Chapter 1}

\section{Introduction}

Many surfaces in nature are superhydrophobic such as lotus leafs, or bird feathers etc.. A drop of water deposited on such a surface adopts the shape of a nearly perfect sphere that rolls off easy, leaving no trace of humidity. Such superhydrophobicity is achieved by combination of two parameters: intrinsic hydrophobicity of the material (wax for example), and roughness or micro-texture. A drop of water deposited on such a rough hydrophobic surface, rests on the crests of the texture. This reduces the actual solid-liquid contact, promoting a spherical drop shape. However a microstructured and hydrophobic surface does not always guarantee mobility of a droplet placed on it. Under certain external conditions (electric field, pressure, temperature, light etc.) the liquid can also invade the texture. Both states result in a rather different behavior of the drop. While a drop resting on crests of the texture will feature a high mobility, providing repellency and self-cleaning effect, a drop which invades the texture is characterized by a low mobility providing no liquid repellency and self-cleaning. It is therefore important to understand the mechanism which of the two states will be adopt on a given surface with a given liquid. Moreover, the ability to switch the droplet from one state to the other will allow us to benefit from the characteristic properties of the states.

Electric field are an excellent tool to control liquids on a small scale. With an electric field, liquid can be actuated in confined geometries such as channels, capillaries [1] or between parallel plates [2]. An electric field can move ionized liquid (electroosmosis), charged particles in a steady liquid (electrophoresis), or 


\section{INTRODUCTION}

neutral particles (dielectrophoresis). On capillary structures like droplets, an electric field can deform the shape by elongating the drop along the direction of the field [3, 4] and an external electrostatic field can be used to move droplets on planar substrates on demand [5]. Finally the electric field also acts on the contact angle of a drop, this effect is called electrowetting.

In following sections we introduce established physical concepts as well as known experimental facts about interfacial aspects of liquids, and liquids on superhydrophobic surfaces. Subsequently, electrowetting as a tool to manipulate liquid interfaces is described. Applied electric fields not only affect the equilibrium shape of a liquid interface, it also gives rise to electrokinetic effects of fluids near solid walls.

\subsection{Surface tension}

If a drop of water is placed on a smooth, clean glass surface (a plate, for example), and another drop on a Teflon frying pan or on greased baking paper, one will be able to see the difference in the behavior of these drops. On the glass the drop flattens out whereas on the Teflon or the greased paper it turns into a ball. We say the drop wets the glass, whereas on a hydrophobic ("water hating") surface, such as Teflon, the wetting is only partial. The spherical shape of a drop is a result of intermolecular forces between the water molecules. A molecule located within the drop is equally attracted in all directions by the molecules surrounding it, and so the total force exerted on it is zero (Fig. 1.1 a). In contrast, at the surface each molecule misses half of its neighbors, i.e. half of its bonds. To bring a molecule to the surface and create an amount of surface area corresponding roughly to its cross section, we have to provide the energy required to break half of its bounds. This energy required to create new surface is the surface energy or surface tension, typically denoted by $\gamma$. Since the sphere has the lowest surface area per given volume, it is easy to understand that this is also the state with the lowest surface energy, and that is what causes the drop to take on a spherical shape.

In different fluids intermolecular forces possess different character and intensity. In organic fluids, such as oil, the attractive forces are a result of momentary 

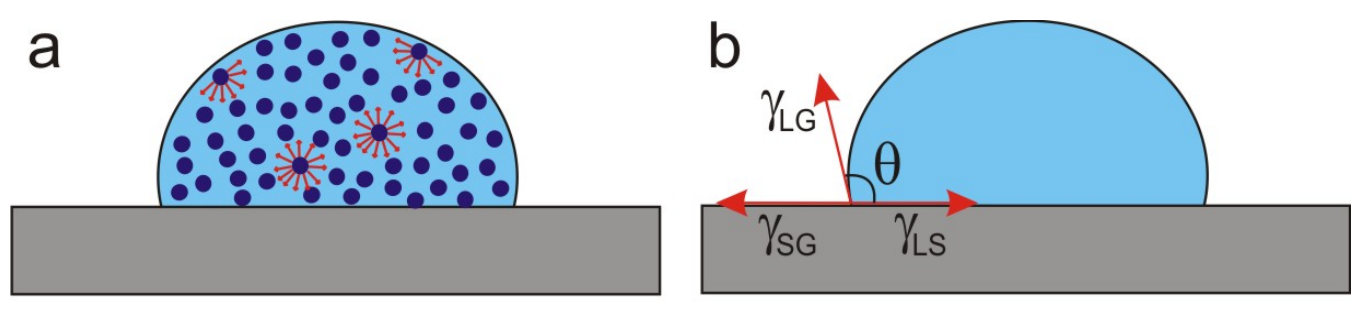

Figure 1.1: (a) The molecules within the drop are equally attracted in all directions, whereas the molecules on the surface of the drop are attracted inward to their neighbors. (b) Surface forces acting on the three phase contact line of a liquid droplet deposited on a substrate

electric polarization of the electrons. This polarization creates a non uniform distribution of electrons in the molecules, and as a result a mutual attraction is created between every two molecules, similar to the attraction between two magnets. The forces responsible for the attraction are called van der Waals forces, after the 19th century Dutch scientist. The surface tension between oil and air resulting from these forces is about $20-50$ millijoule per square meter $\left(\mathrm{mJ} / \mathrm{m}^{2}\right)$. Water is a fluid with many special characteristics resulting from the large permanent dipole of water molecules and their intermolecular hydrogen bonds. Among other things this leads to the relatively high value of surface tension between water and air: $72 \mathrm{~mJ} / \mathrm{m}^{2}$. In mercury, which is a metallic liquid at room temperature, the attractive forces are a result of the free conduction electrons as in solid metals, and the surface tension reaches $485 \mathrm{~mJ} / \mathrm{m}^{2}$. Interfacial tension between two materials depends on their mutual properties, and not just on one of them. For example, the surface tension of a water drop in air is different from the surface tension of that same drop in an oil medium. The noted British physicist Thomas Young, working at Cambridge University, found in 1805 that the contact angle $\theta$ (the angle created between the outer surface of the liquid and the surface on which it lies, see Fig. 1.1 b.) depends on three interfacial tensions: the interfacial tension between the liquid and the solid surface $\gamma_{S L}$, between the surface and the air $\gamma_{S G}$, and between the liquid and the air $\gamma_{L G}$. At equilibrium the three lateral forces acting on the drop are balanced as the drop does not move (see Fig. 1.1 


\section{INTRODUCTION}

b). This force balance can be presented as:

$$
\gamma_{L G} \cos \theta+\gamma_{S L}-\gamma_{S G}=0
$$

The Young equation relates the cosine of the angle $\theta$ to the three surface tensions:

$$
\cos \theta=\left(\gamma_{S L}-\gamma_{S G}\right) / \gamma_{L G}
$$

The two extreme wetting limits are $\theta=180^{\circ}$, which corresponds to a no wetting case, while $\theta=0^{0}$ is the complete wetting case where the liquid spreads uniformly over the surface and creates a thin liquid layer. The intermediate case of $\theta=90^{\circ}$ is achieved when the difference between $\gamma_{S L}$ and $\gamma_{S G}$ of surface tensions in the Young equation 1.2 becomes very small. Of course many other cases are possible with $0<\theta<180^{\circ}$, and those are the ones of interest of this work.

\subsection{Superhydrophobic surfaces}

Lotus leaves are known for their water repellency and consequently to remain clean from any parasitic dust or debris. This phenomenon (also called rolling ball state) is very common in nature not only for the lotus, but also for nearly 200 other species: vegetables, insects and even some animal species. Fig. 1.2 shows a typical Scanning Electron Microscope (SEM) picture of a Lotus leaf.

The common point between all water repellant surfaces is their roughness in combination with low surface energy chemistry. Indeed, the surfaces are composed of micrometric structures limiting the impregnation of the liquid and pushing back the drop. Most of the time, the surfaces contain a second scale of roughness, consisting of nanometric size [7, 8, 9, 10, 11, 12, 13]. In order to minimize its energy, a liquid droplet forms a liquid pearl on the microstructured surface. The superhydrophobicity term is thus used when the apparent contact angle of a water droplet on a surface reaches values higher than $150^{\circ}$. Previously, the studied substrates were regarded as smooth surfaces, i.e. the roughness of the substrate was sufficiently low and thus does not influence the wetting properties 


\subsection{Superhydrophobic surfaces}

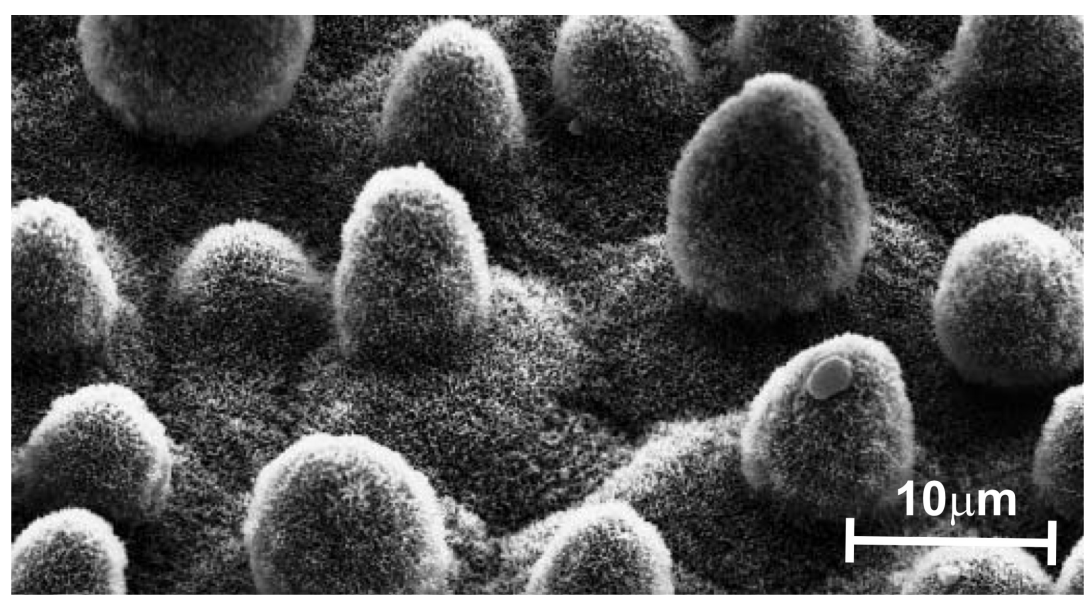

Figure 1.2: SEM image of a Lotus leaf $[6]$

of the surface. In this case, the relation of Young (1.2) gives the value of the contact angle $\theta$ on the surface. However, a surface can have a physical heterogeneity (roughness) or a chemical composition variation (materials with different surface energies). A new contact angle is then observed, called the apparent contact angle noted $\theta^{*}$. It should be noticed that locally, the contact angle between the liquid droplet and the surface can assume almost any value with these geometries due to pinning of the contact line. Two models exist which describe the enhancement of the apparent contact angle: the model of Wenzel [14] and of Cassie-Baxter [15].
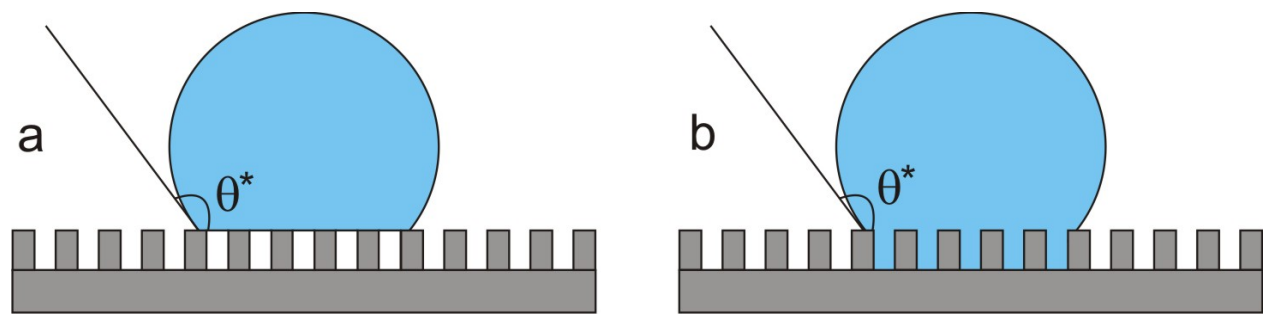

Figure 1.3: Superhydrophobic surfaces: (a) Cassie-Baxter, (b) Wenzel states

A drop on a rough and hydrophobic surface can adopt two configurations: (a) a Cassie-Baxter configuration (air patches are confined below the drop ) and (b) a Wenzel [16] (solid/liquid interface exactly follows the solid roughness) ( 1.3 a 


\section{INTRODUCTION}

and $\mathrm{b}$ ). In both cases an increase in the apparent contact angle $\theta^{*}$ of the drop is observed.
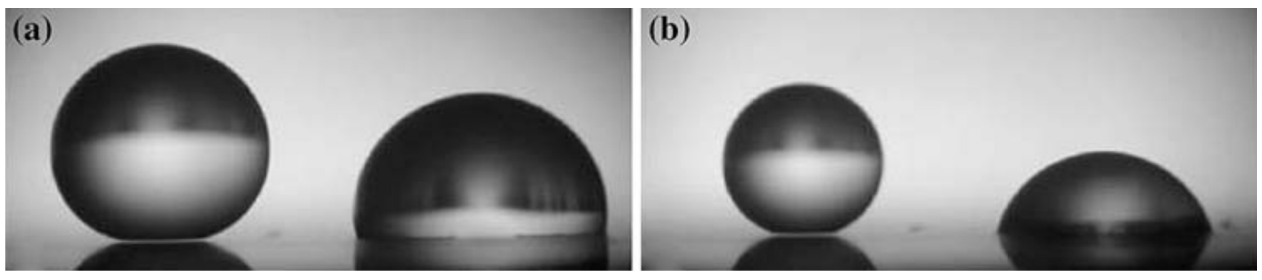

Figure 1.4: Illustration of the difference between the Cassie-Baxter and Wenzel states: (a) after deposition of the liquid drops on the surface, (b) after evaporation [17.

These two models were highlighted by the experiment of Johnson and Dettre [18]. Many research teams have tried to understand in more detail the superhydrophobicity phenomenon and particularly the difficulty of the wetting transition from the Wenzel to Cassie configuration [19]. For a superhydrophobic surface, a pronounced difference between the two models is the hysteresis value. The first experiment on this subject was conducted by Johnson and Dettre (1964) who measured the advancing and receding contact angles, according to the surface roughness [18]. For low roughness, a strong hysteresis, up to $100^{\circ}$ (Wenzel), is observed and attributed to an increase in the substrate surface in contact with the drop. Starting from a certain roughness (not quantified in their experiment), the hysteresis becomes quasi null resulting from the formation of air pockets under the drop (Cassie-Baxter state). The receding angle approaches the advancing angle. Other experiments also show that for a drop in the Cassie-Baxter state, it is possible to obtain a contact angle significantly higher than for a drop in the Wenzel state [17]. The drop on the left in Fig. 1.4 is in a Cassie-Baxter state whereas the drop on the right is in a Wenzel state. After partial evaporation of the drop (Fig. $1.4 \mathrm{~b}$ ), the observed angle (which is the receding angle) is similar to the advancing angle for the drop in the Cassie-Baxter state whereas the drop in the Wenzel state appears like trapped (pinned) on a hydrophilic surface. In the following two paragraphs, we will discuss in detail the two models. 


\subsubsection{Cassie-Baxter state}

Cassie and Baxter did not directly investigate the wetting behavior of liquid droplets on superhydrophobic surfaces. They were more particularly interested in planar surfaces with chemical heterogeneity (Fig. 1.5).

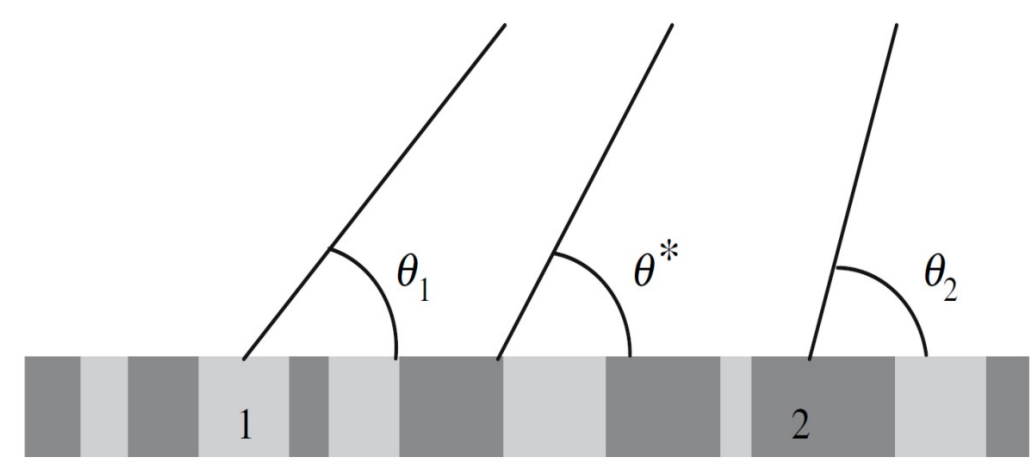

Figure 1.5: Planar surface composed of two different and chemically heterogeneous materials

The examined surface consists of two materials; each one has its own surface energy, characteristic contact angle $\theta_{1}, \theta_{2}$ and occupies a definite fraction of the surface $\phi_{1}$ and $\phi_{2}\left(\phi_{1}+\phi_{2}=1\right)$. We assume that individual areas are very small compared to the size of a drop. Considering a displacement $d x$ of the three phase contact line, the change of energy $d E$ could be expressed by:

$$
d E=\phi_{1}\left(\gamma_{1, S L}-\gamma_{1, S V}\right) d x+\phi_{2}\left(\gamma_{2, S L}-\gamma_{2, S V}\right) d x+\gamma_{L V} d x \cos \theta^{*}
$$

By using the relation of Young, the minimum of $(d E=0)$ leads to the CassieBaxter relation:

$$
\cos \theta^{*}=\phi_{1} \cos \theta_{1}+\phi_{2} \cos \theta_{2}
$$

It is to be noted that the apparent angle $\theta^{*}$ is included in the interval $\left[\theta_{1}, \theta_{2}\right]$.

If material 1 is hydrophobic and material 2 is replaced by air, a drop in contact with each of the two phases (solid and air) forms respective contact angles $\theta$ and 


\section{INTRODUCTION}

$180^{\circ}$, whereas the fractions of respective surfaces are $\phi_{s}$ and $\left(1-\phi_{s}\right)$. Thus, the Cassie-Baxter relation for superhydrophobic surfaces will be:

$$
\cos \theta^{*}=-1+\phi_{s}(\cos \theta+1)
$$

\subsubsection{Wenzel state}

In the Wenzel state, the drop follows the surface and is impaled on its roughness (Fig. 1.3 b). In this case, the solid surface/liquid and solid/gas energies are respectively $r \gamma_{S L}$ and $r \gamma_{S G}$, where the roughness $r$ is defined as the ratio of real surface to the projected surface $(r>1$ for a rough surface area, and $r=1$ for a perfectly smooth surface). A $d x$ displacement of the three phase contact line thus involves a variation of energy:

$$
d E=r\left(\gamma_{S L}-\gamma_{S V}\right) d x+\gamma d x \cos \theta^{*}
$$

At the equilibrium state $(d E=0)$, for a null roughness, i.e. for $r=1$, we find the relation of Young. For a nonnull roughness, the relation of Wenzel [14] is obtained:

$$
\cos \theta^{*}=r \cos \theta
$$

Wenzel's relation embodies two types of behavior:

1. If $\theta<90^{\circ}$, (hydrophilic solid) we will have $\theta^{*}<\theta$ since $r>1$

2. If $\theta>90^{\circ}$, we will have $\theta^{*}<\theta$.

Surface roughness always magnifies the underlying wetting properties. Both hydrophilic and hydrophobic properties of the solid are reinforced by surface topography. Eq1.7 also predicts wetting and drying transitions. Since the roughness $r$ is not bounded, there should exist a threshold value $r^{*}$ beyond which wetting becomes either total or zero, depending on the sign of $\cos \theta$. This threshold value is given simply by $r^{*}=1 / \cos \theta$, is easy accessible. For $\theta=60^{\circ}$, we have $r^{*}=2$. However this statement is highly arguable, the Wenzel's relation is valid only in certain domain of $r$. 


\subsection{Transitions between Cassie-Baxter and Wen- zel states}

All the favorable properties of superhydrophobic surfaces, such as the self-cleaning effect and drag reduction capabilities rely on the Cassie-Baxter state, where the droplet rests on the top of texture. In this state the droplet is highly mobile and can easy roll off from the surface, providing liquid repellency as well as self cleaning effect. In contrast, a drop in the Wenzel state is characterized by a low mobility and high hysteresis. It is therefore is a great interest to understand the mechanism which of the two states will be adopt on a given surface with a given liquid. Moreover, the ability to switch the droplet from one state to another will allow to benefit from both sets of properties characteristic to the states.

These states are (meta)stable states, which mean that a drop of water can stay in one of these states infinitely long if there is no external factor which changes the free energy of the drop. This also means that by changing the free energy of the droplet it is possible to switch from one state to another. However, a reversible transition from the Wenzel to Cassie-Baxter states is normally very complicated to achieve because of the existence of (Gibbs) energy barriers between the states [20, 21]. In particular, it is the transition from the Wenzel to the Cassie-Baxter state that proves problematic because the base of the liquid drop in the Wenzel state cannot detach from the solid surfaces. There are several approaches reported in literature to achieve a reversible transition between Cassie-Baxter and Wenzel states: mechanical [22, 23, 24], magnetic [25, 26], chemical [27], temperature assisted [28, 29], optical [25, 30, 31, 32], electrical [33, 34]. Howewer, in many of mentioned works only the properties of the surface that are switched from superhydrophobic to not superhydrophobic. It is more important the ability to switch a given drop from one state to another. A promising method of reversibly switching between Cassie-Baxter to Wenzel states is electrowetting, especially for Lab-on-Chip applications. Although electrowetting induced transition from Cassie-Baxter to Wenzel state is rather straightforward [35]. Krupenkin in 2007 demonstrated the first solution for the reversible wetting on such surfaces [33. A very short electrical current impulse applied to the substrate leads to surface heating. The temperature can then reach $240^{\circ} \mathrm{C}$, causing liquid boiling 


\section{INTRODUCTION}

and droplet expelling from the surface. Even though this technique is easy to implement, it is hard to imagine such an integrated system within a Lab-on-Chip for example. The heating would cause significant damage to biological material within the drop. Moreover, this expulsion creates satellite droplets.

Other teams worked on electrowetting on textured surfaces by using various materials, like SU-8 [36] or carbon nanotubes (CNT) [34]. In the first case, the reversibility is only partial. The angle decreases from $152^{0}$ to $90^{\circ}$ under $130 \mathrm{~V}$ and returns to $114^{0}$ when the voltage is cut off. In the second case (CNT), no reversibility is observed. A solution allowing the reversibility is to modify the surrounding medium. Indeed, the irreversibility is observed when the ambient medium is air. By replacing air by a hydrophobic medium, like oil (dodecane), it is possible to obtain reversibility. The angle decreases from $160^{\circ}$ to $120^{\circ}$ when a voltage was applied and returns back to $160^{\circ}$ after voltage cut off [34].

\subsection{Liquids in an electric field}

The influence of electric forces on liquid structures can be observed in a simple "bathroom experiment" when a plastic rod (a hair brush) charged by friction on clothes is approached to a flowing liquid filament. The interaction between the charges in the liquid and the charges at the surface of the plastic rod results in a force which bends the interface. Using an electric field, liquid can be actuated in confined geometries such as channels, capillaries [1] or parallel plates [2]. An electric field can move ionized liquid (electroosmosis), charged particles in a steady liquid (electrophoresis), or neutral particles (dielectrophoresis). On capillary structures like droplets, an electric field can deform the shape by elongating the drop along the direction of the field [3, 4] and an external electrostatic field can be used to move droplets on planar substrates on demand [5]. Finally the electric force also acts on the contact angle of a drop, this effect being named electrowetting (more precisely electrowetting on dielectric). The so called electrowetting effect is linked to dielectrophoresis as shown in [2] and has already been used to actuate liquid in, in particular to reach droplet motion [37], switch between droplet morphologyes [38] or actuation in confined systems [39]. 
In this thesis we mainly focus on two aspects of electric field-liquid interaction: electrowetting and electrodiffusion.

\subsubsection{Electrowetting}

The basis of electrowetting has been first described more than a century ago by a French physicist named Gabriel Lippmann who investigated effects of electrocapillarity which laid the basis of modern electrowetting (for english translation Lipmann's work see [40]). For more details on Lippmanns work and electrowetting in general, see [40, 41]. Based on his findings, a term due to electric polarization was added to the Young equation. This generalized equation is called the Young-Lippmann equation:

$$
\gamma_{L G} \cos \theta_{E}=\gamma_{S G}-\gamma_{S L}+1 / 2 C U^{2}
$$

where $U$ is the electric voltage and $C$ is the electric capacitance per unit area in the region of contact between a conducting surface and an electrolyte drop separated by an insulating layer. Taking into account Young's equation 1.2 we can rewrite 1.8 in following form:

$$
\cos \theta_{E}=\cos \theta+\frac{\varepsilon_{o} \varepsilon_{l}}{2 d_{H} \gamma} U^{2}
$$

where $\gamma$ is the surface tension of the liquid, $\varepsilon_{0}$ is vacuum permittivity, $\varepsilon_{l}$ and $d_{H}$ are respectively, the dielectric constant and the thickness of the insulator. The second term in the right hand side is known as the electrowetting number,

$$
\eta=\frac{\varepsilon_{o} \varepsilon_{l}}{2 d_{H} \gamma} U^{2}
$$

which measures the strength of the electrostatic energy compared to surface tension.

To understand how the contact angle reduction is achieved in mechanical terms one should consider the forces exerted on the liquid by the electric field. Consider a droplet sitting on a flat dielectric-coated electrode with a voltage applied between the liquid and the electrode, as shown in Fig. 1.6 b. Assuming the liquid near the solid/liquid boundary possesses a net charge such that the 


\section{INTRODUCTION}
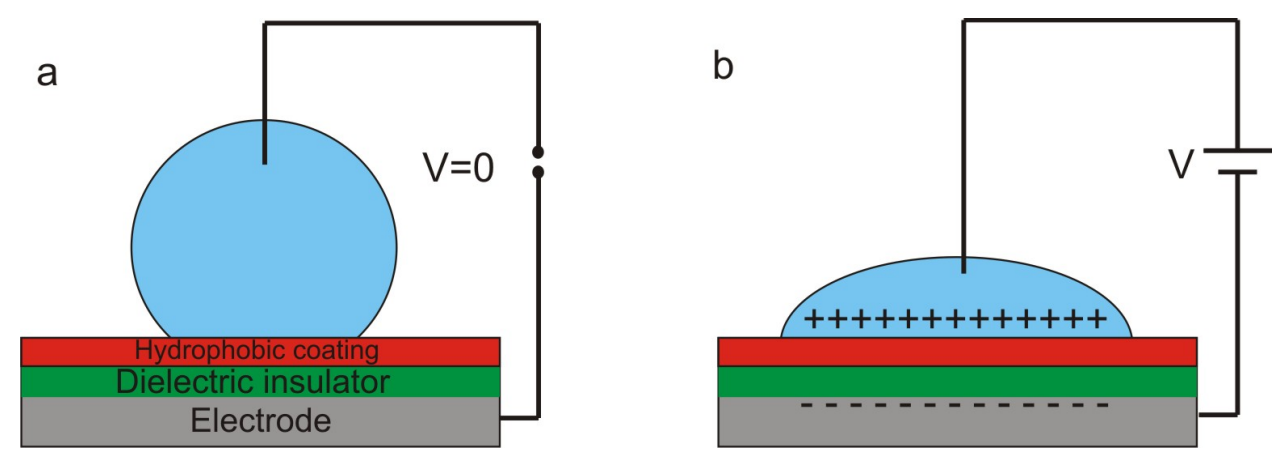

Figure 1.6: (a) A water drop placed on a hydrophobic surface with a high contact angle. (b) Electrowetting of the surface. Operation of voltage between the drop and the electrode changes the distribution of charges due to the dielectric insulator and significantly decreases the contact angle. The two surface coatings are drawn not to scale.

field is completely screened from the interior of the liquid, the droplet will feel electrostatic pressure acting in the normal direction at every point on its interface with solid. The fringing electric field formed at the rim of the droplet exerts electrostatic pressure on the liquid-gas boundary right above the contact line and hence a net force in the direction parallel to the solid, causing droplet spreading. From this force one may formulate an electromechanical problem without assuming that the solid-liquid surface tensions are changed by external voltage. For example, by integrating the Maxwell stress tensor over a control surface around the liquid-fluid boundary, Jones showed that total force per unit length of contact line is equal to $C V^{2} / 2$, demonstrating electromechanical derivation of the electrowetting equation [42. Jones derivation required no information about the actual shape of the liquid-fluid interface. Use of the Maxwell stress tensor for calculating the various forces on conductive and dielectric liquid droplets is covered in-depth by Zeng and Korsmeyer [43].

Use of this approach is particularly important for determinarion of the local morphology of the drop surface. The local morphology of the drop surface however does depend on the distribution of the electric field and of electric charges in the system. As noted above, we assume that the liquid is perfectly conductive. Hence, $\boldsymbol{E}$ inside the drop and $(\boldsymbol{E} \boldsymbol{t})_{\text {surf }}=0$, i.e. the electric field is oriented 
perpendicular to the surface ( $\boldsymbol{t}$ is a local tangent vector at the drop surface). In this case the electric field gives rise to a Maxwell stress

$$
\Pi_{e}(\boldsymbol{r})=\frac{\varepsilon_{0}}{2} \boldsymbol{E}(\boldsymbol{r})^{2}
$$

pulling on the liquid surface along the outward normal. The drop surface is in mechanical equilibrium if electrostatic Maxwell stress is balanced by the Laplace pressure:

$$
\Pi_{e}(\boldsymbol{r})=\gamma\left(\frac{f^{\prime \prime}}{\left(1+f^{\prime 2}\right)^{3 / 2}}\right)
$$

Solving equation 1.12 for the drop shape requires the exact distribution of the electric field, which itself depends on the drop shape. Hence both the drop shape and the electric field distribution have to be calculated in a self-consistent manner. Buehrle et al. [? ] developed iterative technique to calculate equilibrium surface profiles near the three phase contact line.

In Chapters $\mathbf{2}$ and $\mathbf{4}$ we use this approach to study possible mechanisms for the transition from the Cassie-Baxter state to the Wenzel state on superhydrophobic surfaces under the influence of electric fields both numerically and experimentally.

\subsubsection{Electrodiffusion}

Another example of electric field-liquid interaction is electrodiffusion, which is a nonlinear transport process whose essence is the diffusion of charged particles together with their drift in a self-consistent electric field. It is in fact the diffusion in a "preferred" direction which follows from the fact that the electric field induces a force on a charged particle balanced by the effective force of friction due to collisions with other solute or solvent particles. Basic equations of electrodiffusion were obtained about 120 years ago by Nernst and Planck in application to the motion of ions. About 60 years ago Van Roosbroeck [44] used these equations to treat the transport of holes and electrons in semiconductors. Most applications of the theory of electro-diffusion relate to electrophysiology, electrochemistry and 


\section{INTRODUCTION}

chemical and electrical engineering. These are concerned, respectively with nervous conduction, ion separation, electric energy production, desalination of saline water, and semiconductor device technology.

Passage of an electric current through a solution adjacent to an ion-selective interface results in the formation of an electrolyte concentration gradient. This effect is called concentration polarization 45 . The expression of concentration polarization is the typical nonlinear steady-state voltage/current (VC) dependence, schematically depicted in Fig. 1.7. The following three regions are distinguishable in such a curve: linear (Ohmic) Region I is followed by current saturation in Region II (limiting current), which is in turn followed by inflexion of the VC curve and transition to "over limiting" conductance regime (Region III), accompanied by the appearance of low-frequency excess electric noise.

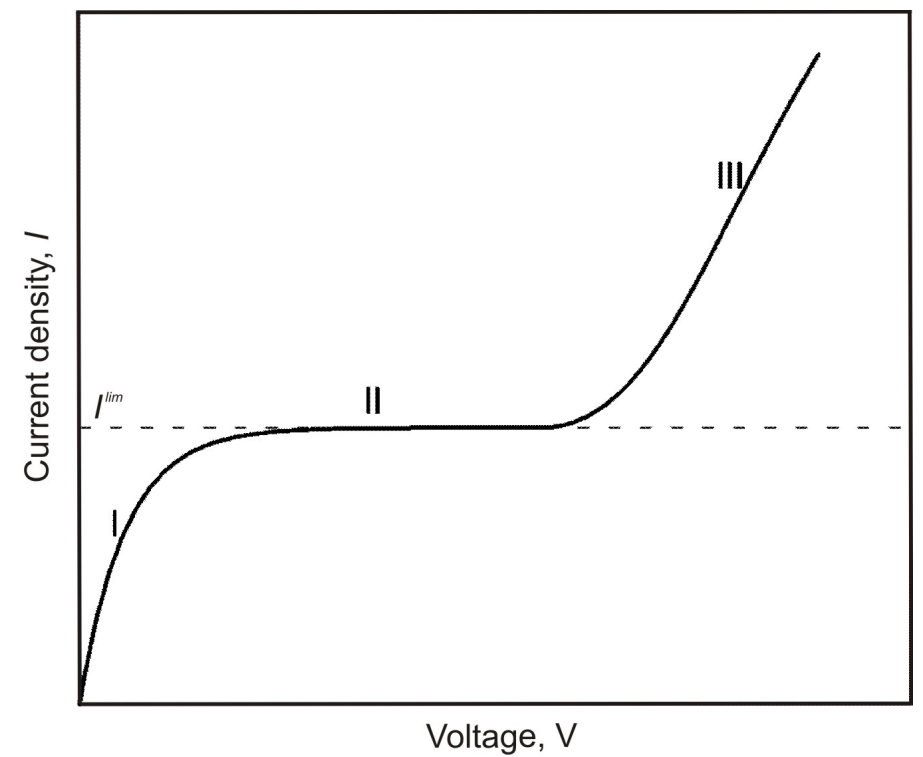

Figure 1.7: Sketch of a typical voltage current curve of a cation-exchange membrane

For a given flow, polarizability of a perm-selective ion-exchange membrane by a DC current is determined by geometric factors, such as, the typical size of the ion-permeable "gates" at the membrane surface in relation to the separation distance between them and the diffusion layer thickness. On the other 
hand, the major factor determining the value of the limiting current through any charge-selective solid is hydrodynamics. Thus the limiting current may be increased by increasing the flow velocity past the membrane, thereby reducing concentration polarization. The source of this effect becomes particularly clear if one distinguishes between the bulk of the fluid where the solute transport is entirely dominated by convection, and the diffusion boundary layer (often called "unstirred" or Nernst layer) where the transport is electrodiffusion dominated. The classical theory of concentration polarization predicts a true saturation of the
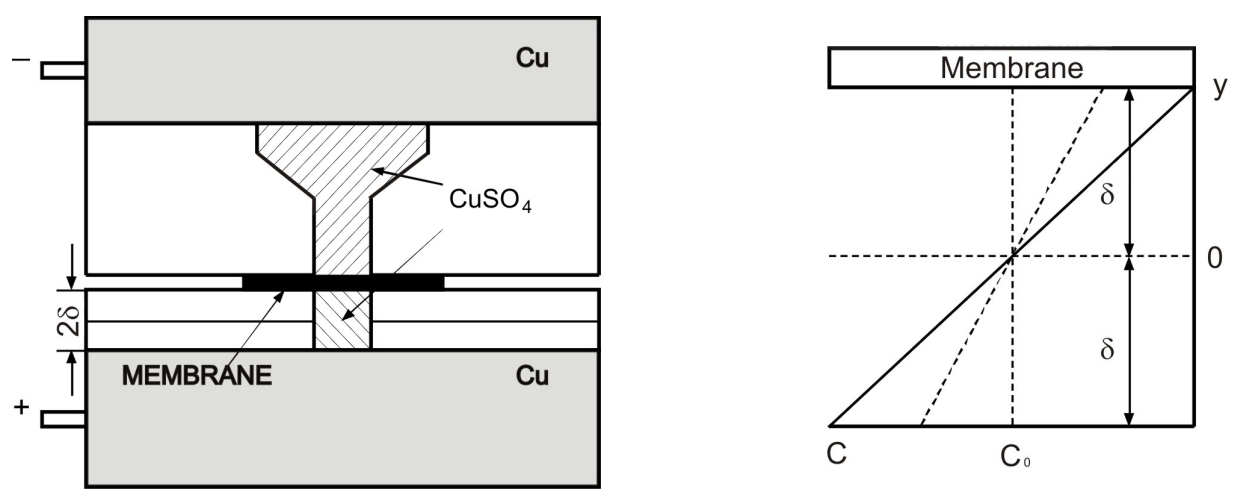

Figure 1.8: Sketch of quiescent polarization cell and steady-state electrolyte concentration polarization in it.

VC curves at the limiting current, offering no explanation for the "over-limiting" conductance [45. For definiteness, consider an "unstirred" layer of thickness $\delta$ of a univalent electrolyte adjacent to an ideally perm-selective homogeneous interface (e.g., a cation-exchange membrane) (Fig. 1.8). Let us direct the axis $y$ normally to this interface, with the origin at the membrane-solution interface and $y=-\delta$ coinciding with the outer (bulk) edge of the "unstirred" layer. Let us assume local electroneutrality and neglect the electroosmotic flow. With these assumptions, stationary ionic transport across the "unstirred" layer will be described by the following boundary value problem:

$$
\begin{gathered}
D\left(\frac{d c}{d y}+\frac{F}{R T} c \frac{d \phi}{d y}\right)=-j=-\frac{i}{F} \\
\frac{d c}{d y}-\frac{F}{R T} c \frac{d \phi}{d y}=0
\end{gathered}
$$




\section{INTRODUCTION}

$$
\begin{aligned}
& c(-\delta)=c_{0} \\
& \phi(-\delta)=0 \\
& \phi(0)=-V
\end{aligned}
$$

Here, $y, c, \phi, j, i$ and $V$ are, respectively, the dimensional coordinate, ionic concentration, electric potential, cation flux, electric current density, and voltage drop across the "unstirred" layer, whereas $c_{0}$ is the bulk concentration, $F$ is the Faraday constant, $R$ is the universal gas constant, $T$ is the absolute temperature, and $D$ is the cation diffusivity. Equations 1.13 and 1.14 are the stationary NernstPlank equations for electrodiffusional transfer of cations and anions, respectively. Integration of 1.13 1.17 yields:

$$
\begin{gathered}
c=c_{0}\left[1-\left(\frac{j}{2 D c_{0}}\right)(y+\delta)\right] \\
\phi=\frac{R T}{F} \ln \left[1-\left(\frac{j}{2 D c_{0}}\right)(y+\delta)\right] \\
i=\frac{2 F D c_{0}}{\delta}\left(1-e^{-\frac{V F}{R T}}\right)
\end{gathered}
$$

For, $V \rightarrow \infty$ Eq. 1.20 predicts

$$
i \rightarrow i^{\lim }=\frac{2 F D c_{0}}{\delta}
$$

Let us note that for $V=O(1)$ and $i<i^{l i m}$ the structure of diffusion layer is characterized by the splitting into quasi-electro-neural bulk and a thin boundary layer: quasi-equilibrium electric double layer. This picture breaks down upon $i \rightarrow$ $i^{l i m}$ as reflected, in particular, in the inconsistency of the local electroneutrality approximation, which appears in the basic concentration polarization solution 1.18, 1.19. For $V>>O(1)$ and $i \rightarrow i^{l i m}$ quasi-equilibrium electric double layer expands and transforms into non-equilibrium electric double layer characterized by the presence of the extended space charge region [46]. 
As shown in [46, 47, 48, the development of the non-equilibrium electric double layer with the extended space charge region has a strong effect on various electrokinetic phenomena and results in the appearance of non-equilibrium electrokinetic phenomena such as electrophoresis of the second kind, electroosmotic slip of the second kind et.c. In particular, non-equilibrium electroosmotic slip instability and resulting electroconvection in concentration polarization can break current saturation as shown in [46]. Thus, the study of the electric double layer dynamics at the limiting current, in particular, its transition from quasiequilibrium to non-equilibrium regimes, is an essential component of the general study of non-equilibrium phenomena in electrodiffusion.

The Chapter 6 of this thesis is dedicated to experimental study of electroosmotic instabilities near the charge selective membrane.

\subsection{Outline of the thesis}

This thesis is presented in six chapters. Chapter $\mathbf{1}$ presents a brief introduction to the wetting phenomenon, liquid behaviour on superhydrophobic surfaces. Then we discuss electrowettnig and electrodiffusion as a tool to manipulate liquids at the interfaces. In Chapter 2 We discuss the equilibrium shape of the composite interface between superhydrophobic surfaces and drops in the superhydrophobic Cassie-Baxter state under upplied electric field. We demonstrate that the equilibrium shape of the interface is determined by the balance of the Maxwell stress and the Laplace pressure. Energy barriers due to pinning of contact lines at the edges of the hydrophobic pillars control the transition from the Cassie to the Wenzel state.

As a natural follow up to the Chapter 2 in Chapter 3 we present two approaches for electrowetting induced reversible transitions between the CassieBaxter to the Wenzel states. We show how the electrowetting effect can be used for achieving locally switching between the two wetting states using suitable surface and electrode geometries.

In Chapter 4 we study possible mechanisms for the transition from the Cassie-Baxter state to the Wenzel state on superhydrophobic surfaces under the influence of electric fields as a function of the aspect ratio and the wettability 


\section{INTRODUCTION}

of the surface, both numerically and experimentally. Fully self-consistent calculations of both electric field distribution and surface profiles show that this instability evolves from a global one towards a local Taylor cone-like instability for increasing aspect ratio of the cavities, which were confirmed with our experimental results

In Chapter 5 we investigate the wetting phenomenon of a drop on a sphere geometry. For this geometry we confirmed the predictions from free energy calculations with experimental results an determined under which conditions the droplet will wet the sphere.

Finally in Chapter 6 we present the first direct experimental visualization of a theoretically predicted hydrodynamic instability of ionic conduction from a binary electrolyte into a charge selective solid. At steady state, upon the passage a DC current, current/voltage dependence exhibits a characteristic saturation at the limiting current. Upon a further increase of voltage, current increases marking the transition to the overlimiting conductance regime. We show that this transition is mediated by the appearance of a vortical flow that increases with the applied voltage in the overlimiting regime. 


\section{Chapter 2}

\section{Electrical switching of wetting states on superhydrophobic surfaces}

\subsection{Abstract}

In this chapter we demonstrate that the equilibrium shape of the composite interface between superhydrophobic surfaces and drops in the superhydrophobic CassieBaxter state under electrowetting is determined by the balance of the Maxwell stress and the Laplace pressure. Energy barriers due to pinning of contact lines at the edges of the hydrophobic pillars control the transition from the Cassie to the Wenzel state. Barriers due to the narrow gap between adjacent pillars control the lateral propagation of the Wenzel state. For large pillar spacing, the Wenzel state propagates over the entire drop-substrate interface, for small spacings inhomogeneous partially collapsed states can be obtained.1 $]^{1}$

\footnotetext{
${ }^{1}$ Part of this chapter has been published in "Electrical Switching of Wetting States on Superhydrophobic Surfaces: A Route Towards Reversible Cassie-to-Wenzel Transitions", G. Manukyan, J. M. Oh, D. van den Ende, R. G. H. Lammertink, and F. Mugele [49].
} 


\section{ELECTRICAL SWITCHING OF WETTING STATES ON SUPERHYDROPHOBIC SURFACES}

\section{$2.2 \quad$ Introduction}

Superhydrophobic surfaces display remarkable properties including ultrahigh contact angles, ultralow contact angle hysteresis [50], large hydrodynamic slip [51, 52, 53], and tunable optical diffraction [54, 55]. These properties rely on the weak interaction between the liquid and the substrate due to the entrapment of air (or vapor) in the cavities of the rough surface topography. At the transition from the superhydrophobic "Cassie" state to the normal "Wenzel" wetting state, the gas from the cavities is expelled and the interaction between the liquid and the substrate increases dramatically. The contrasting properties of the Cassie and the Wenzel state make it particularly attractive to design surfaces that allow for switching between the two wetting states. Various external control parameters have been used to trigger the transition from the Cassie to the Wenzel state, including hydrostatic pressure, optical and chemical stimuli (see [56] for a review), as well as electric fields [33, 34, 35, 36, 57, 58]. In particular, the latter offers opportunities for fast and precise fine-tuning of the wetting state. Yet, a microscopic understanding of the mechanism controlling the properties of the superhydrophobic state under electrowetting (EW) conditions and in particular the stability limit of the Cassie state is lacking [58, 59]. Such a detailed understanding will be crucial to reach the holy grail of reversible switching between these states, which has so far been limited to a few special cases involving partial evaporation [33], violent mechanical shaking [60], and specific water-in-oil systems [34].

In this chapter we analyze the properties of liquid drops on superhydrophobic surfaces consisting of periodic arrays of micrometer-sized posts under electrowetting. Using reflection contrast interference microscopy, we determine the deflection of the composite water-air interface under the influence of an applied voltage. We identify the dimensionless parameters that control the reversible deflection of the interface at low voltage and we show that the critical voltage for inducing the Cassie-to-Wenzel transition is determined by the depinning of the three-phase contact line from edges at the top of the posts. 


\subsection{Materials and methods}

\subsubsection{Sample preparation}

Superhydrophobic surfaces were prepared in cleanroom using photolithography technique. ITO covered glass was spin-coated with $5 \mu m$ thick SU-8 3005 photoresist, then exposed with UV light and developed. This provides an homogenous layer of a dielectric (thickness $d=5 \mu \mathrm{m}$; dielectric constant $\varepsilon=3.2$ ), which prevents the samples from breakdown when under electric field the microcavities are filled with water. On top of that homogeneous layer the microstructure was made, square lattices of round pillars (diameter $2 a=5 \mu \mathrm{m}$; height $h=5 \mu \mathrm{m}$ distance between the pillars $d=3,5,8$ and $10 \mu \mathrm{m}$ ). Note that the geometry chosen here resembles the one of [36] rather than [33, 35, 58]. In the latter case, the electric field acts as a means to reduce the contact angle, whereas in the present experiments it acts everywhere along the micromenisci at the drop-substrate interface. The corresponding values of Wenzel surface roughness factor $r=A_{\text {real }} / A\left(A_{\text {real }}\right.$ : true solid surface area following the topography; $A$ : projected surface area) and of the fractional area $f$ of the pillars are given in Table 2.1. The surfaces are hydrophobized by dip-coating from a $0.01 \%$ Teflon AF (Dupont) solution, which preserves the original pillar geometry (see Fig 2.13) and produces a contact angle on the flat surface area next to the pillar array of $\theta_{Y} \approx 115^{0}-120^{0}$.

\begin{tabular}{ccc}
$d(\mu m)$ & $r$ & $f$ \\
\hline \hline 3 & 2.23 & 0.307 \\
5 & 1.79 & 0.196 \\
8 & 1.46 & 0.116 \\
10 & 1.35 & 0.087
\end{tabular}

Table 2.1: Sample characteristics. $r$ : Surface roughness, $f$ : fractional area.

\subsubsection{Experimental setup}

The experimental setup consists of a millimeter-sized sessile drop (DI water) on a superhydrophobic surface as shown in Fig 2.1b. Inserting the values of $\theta_{Y}, r$ 


\section{ELECTRICAL SWITCHING OF WETTING STATES ON SUPERHYDROPHOBIC SURFACES}

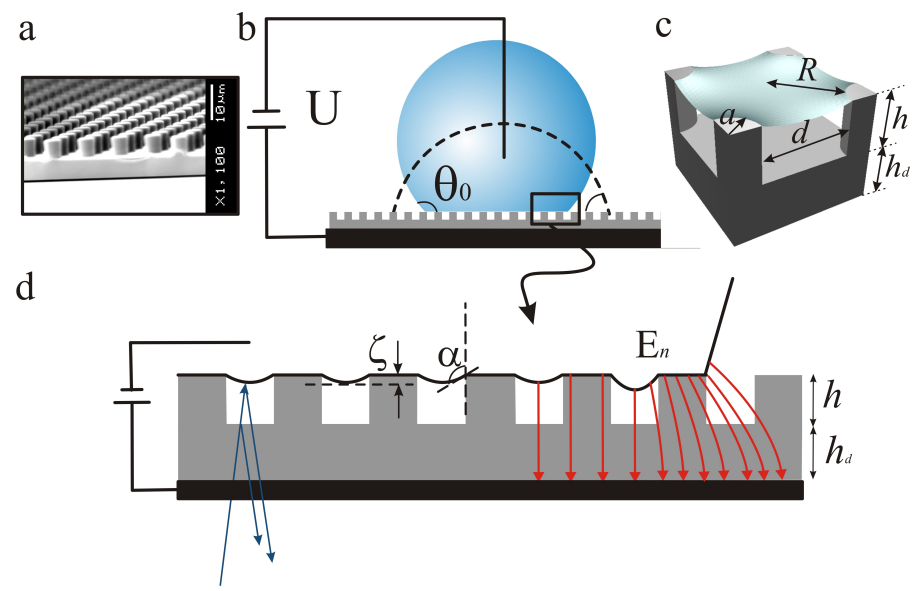

Figure 2.1: (a) System configurations. (a) SEM image of Teflon coated microstructure. (b) Electrowetting setup: a voltage $U$ is applied between a droplet and an electrode covered with a dielectric micro-patterned surface. The contact area is monitored with an inverted microscope from the bottom through the superhydrophobic substrate. (c) The unit cell of microstructure. (d) Magnified sketch of the solid-liquid interaction area. Blue arrows: optical rays leading to interference. Red arrows: electric field distribution (shown only close to contact line).

and $f$ into the standard expressions [50] for the energies $G_{C}$ and $G_{W}$ per unit area of the Cassie and the Wenzel state, respectively, shows that $\left(G_{C}-G_{W}\right) / \sigma=$ $(r-f) \cos \theta_{Y}+(1-f)$ is positive, i.e. that the Wenzel state is thermodynamically favorable, for all samples except for the smallest pillar spacing. As usual in EW experiments, the macroscopic drop shape is controlled by applying a voltage (here: DC) between the drop via an immersed metal wire and the ITO electrode. The drop-substrate interface is illuminated from below with monochromatic light $(\lambda=510 \mathrm{~nm})$ and observed in reflection mode using an inverted microscope.

\subsection{Observations}

Upon linearly increasing the applied voltage the drop spreads and the (except for some faceting for closely spaced pillars) circular drop-substrate interfacial area increases (see Fig, 2.2). In the reflected light, the SU-8 pillars appear dark since the illuminating light is largely transmitted through the polymer-water interface 


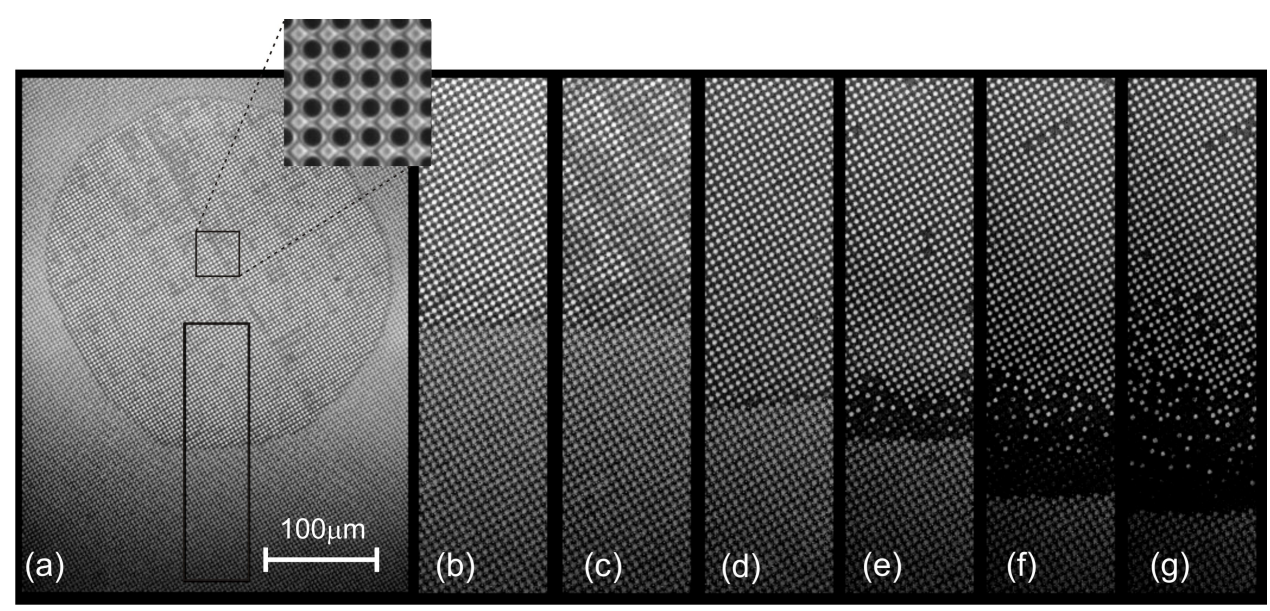

Figure 2.2: Spreading of droplet on the superhydrophobic surface by ramping the voltage $(d=5 \mu \mathrm{m})$. Voltage increases from left to right : (a) $0 \mathrm{~V}$ the Cassie state, the entire contact area is shown: the droplet touches the tops of the microscopic pillars, (b)-(g) Magnified views of the black rectangle region in (a) at $U=0 \sim 250 \mathrm{~V}$ for every $50 \mathrm{~V}$, respectively. Black dots appeared near the contact line in (d)-(g) represent the area in the Wenzel state. Inset: magnified view of the solid-liquid contact area at maximal deflection of menisci.

(due to near index-matching conditions), whereas the air-filled area in between appears bright with some lateral intensity variations due to interference of the light that is partially reflected at the solid-air and at the liquid-air interface, respectively (see Fig 2.1b and inset of Fig,2.2). As the voltage increases, the interference pattern changes reversibly.

At a certain critical value, the area between some of the pillars along the edge of the drop, i.e. along the macroscopic apparent contact line, turns dark, indicating that these pits have been filled with water, as shown in Fig 2.2 . The subsequent behavior depends strongly on the pillar spacing. For the smaller spacings ( 3 and $5 \mu \mathrm{m}$ ), more and more individual pits turn dark one after the other (Fig.2.2 and g) while neighboring pits can remain air-filled. The drop-substrate interface is then inhomogeneous displaying a Wenzel-like state with water-filled pits along the apparent contact line and a Cassie-like state with entrapped air in the central region. In contrast, for the larger pillar spacings the region with water-filled pits spreads within $\sim 2-3 m s$ across the drop-substrate interface leading to a homoge- 


\section{ELECTRICAL SWITCHING OF WETTING STATES ON SUPERHYDROPHOBIC SURFACES}

neous Wenzel state except for typically one air bubble in the center that dissolves on a longer time scale. Occasionally, for intermediate pillar spacings of $5 \mu \mathrm{m}$, we also observed a mixed scenario: pits in the neighborhood of already filled pit have a higher probability of becoming filled. Yet, rather than spreading homogenously over the entire drop-substrate interface, branched lines and occasionally clusters of collapsed pits appear (see Fig2.3a).
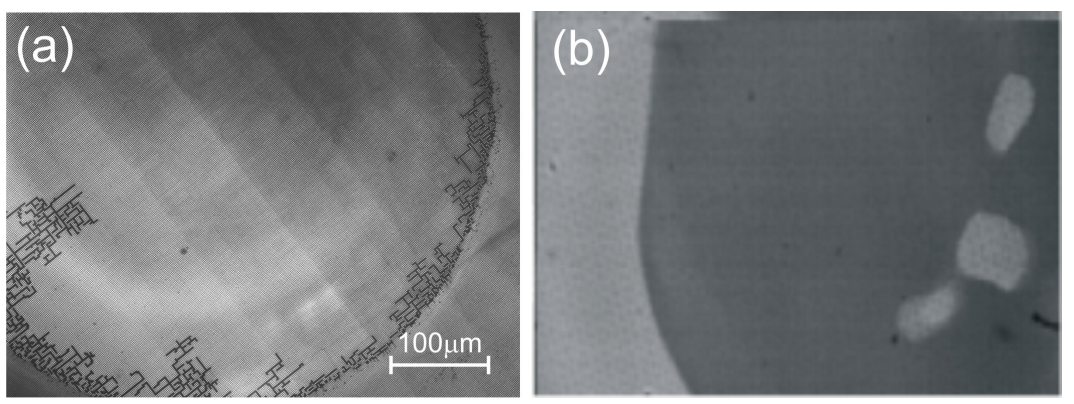

Figure 2.3: Final state after transition to Wenzel state. (a) $(d=3 \mu m)$ : heterogeneous drop substrate interface with linear structures of dark filled pits. (b) $(d=10 \mu m)$ : homogeneous Wenzel state, except for three bright bubble (right).

To study the Cassie-to-Wenzel transition in more detail, we analyze the interference pattern originating from the deformable water-air interface. The interference pattern in the inset of Fig 2.2 indicates that the deflection of the interface is maximal in the center of the pits between four neighboring pillars. In between two adjacent pillars, the surface displays a saddle point. To quantify the deformation of the interface, we extract the intensity averaged over the central area of typically 9 adjacent cavities as a function of the applied voltage, (see Fig 2.4). As long as the applied voltage does not exceed the critical voltage $U_{c}$, the interface reversibly bends up and down indicating that the microscopic three-phase contact lines along the edges of the pillars remain rigidly pinned. From the number of maxima and minima of the interference pattern, it is obvious that the maximum deflection is less than twice the wavelength of the incident light. Given the spacing between the pillars, this implies that the overall deformation of the water-air interface remains moderate. Thanks to the resulting small slopes, we can determine the deflection $\zeta_{0}$ of the water-air interface simply by considering 
the interference of plane waves [61]: As shown in Fig 2.4 b, the resulting deflection increases with increasing voltage up to $U_{c}$, where the system undergoes the transition to the Wenzel state. As intuitively plausible, for any fixed voltage the deflection increases and $U_{c}$ decreases with increasing pillar spacing (see Table 2.1.).
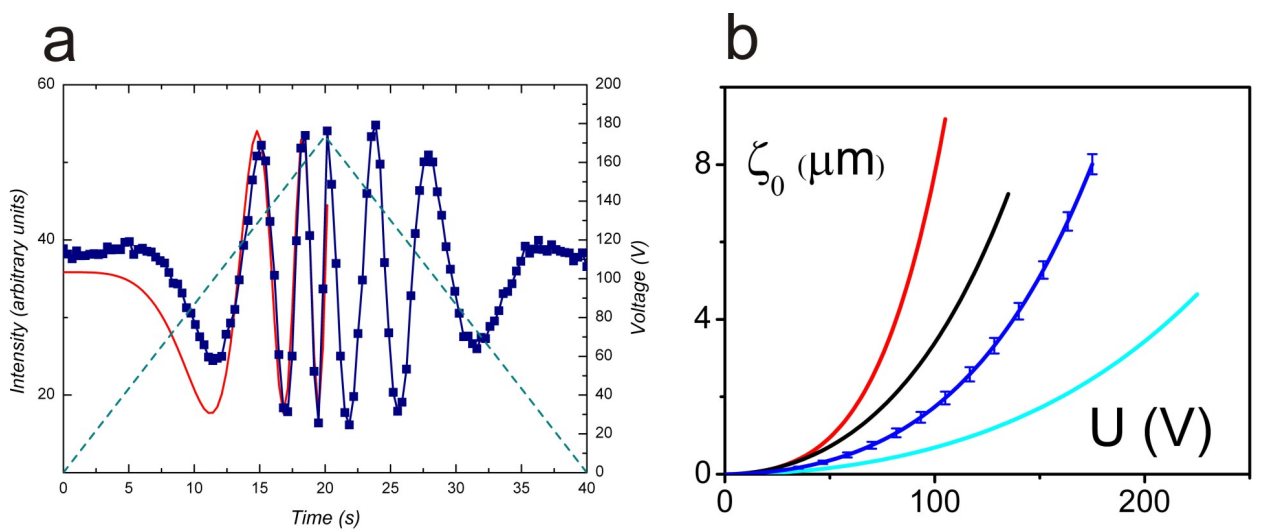

Figure 2.4: a: Variation of the reflected light from micropits during the linear voltage ramp from 0 to $175 \mathrm{~V}$ and back to 0 , for distance between posts $d=5 \mu \mathrm{m}$. b: maximal deflection of the meniscus as a function of applied voltage for $d=10,8,5$ and $3 \mu m$ 


\section{ELECTRICAL SWITCHING OF WETTING STATES ON SUPERHYDROPHOBIC SURFACES}

\subsection{The model}

The deflection of the interface is due to the electric field $\vec{E}$ and the resulting Maxwell stress $\pi_{e l}=\varepsilon \varepsilon_{0} E^{2} / 2$ ( $\varepsilon_{0}$ : vacuum permittivity; $\varepsilon$ : relative permittivity of medium in cavities in Cassie state, i.e. $\varepsilon=1$ for air) pulling outwards along the surface normal. In mechanical equilibrium, $\pi_{e l}$ is locally balanced by the Laplace pressure $\Delta p_{L}=\sigma \kappa$ of the curved interface, where $\sigma$ is the surface tension and $\kappa$ the mean curvature of the water-air interface:

$$
\pi_{e l}(x, y)=p_{L}(x, y)
$$

Denoting the local deflection of the water-air interface away from the flat configuration as $\zeta(x, y)$, the mean curvature in small slope approximation is given by $\kappa=\partial^{2} \zeta / \partial x^{2}+\partial^{2} \zeta / \partial y^{2}$. Since the electric field distribution depends on the shape of the water-air interface, finding the equilibrium shape requires in principle a self-consistent calculation of both field distribution and surface profile, as for instance in the case of surface profiles close to the three-phase contact line in EW [62]. Given the small slopes of the water-air interface, the present electrical problem can be simplified assuming a locally flat configuration with a positiondependent local deflection resulting in an electric field $E(x, y)=U / z_{\text {eff }}$ at the liquid surface, where $z_{e f f}=H-\zeta(x, y)$ with $H=h+h_{d} \varepsilon / \varepsilon_{d} \approx 6.5 \mu m$, is the effective dielectric spacing between the water-air interface and the electrode. We rescale the $x$ and $y$ coordinates by the radius $R=\sqrt{2}(d / 2+a)-a$ (see Fig 2.1c) writing $x=u R$ and $y=v R$ and the vertical coordinate $z$ by the effective thickness $H$ writing $z=w H$. Eq2.1 then assumes the dimensionless form:

$$
\frac{\partial^{2} w}{\partial u^{2}}+\frac{\partial^{2} w}{\partial u^{2}}=\frac{\Lambda^{2}}{w^{2}}
$$

where $\Lambda=R / H \eta^{1 / 2}$ with $\eta=\varepsilon \varepsilon_{0} U^{2} / 2 H \sigma$ is a dimensionless number measuring the relative strength of electrostatic and surface tension forces. Note that $\Lambda$ is given by the square root of the usual EW number $\eta$ (see e.g. [40]) modified by the ratio of the lateral over the vertical length scale.

Eq2.2 can be solved numerically using periodic boundary conditions with a quadrant of the unit cell with $u, v \in[0,(a+d / 2) / R]$ and a finite differences relaxation scheme. The boundary conditions read $w=1$ along the edge of the 

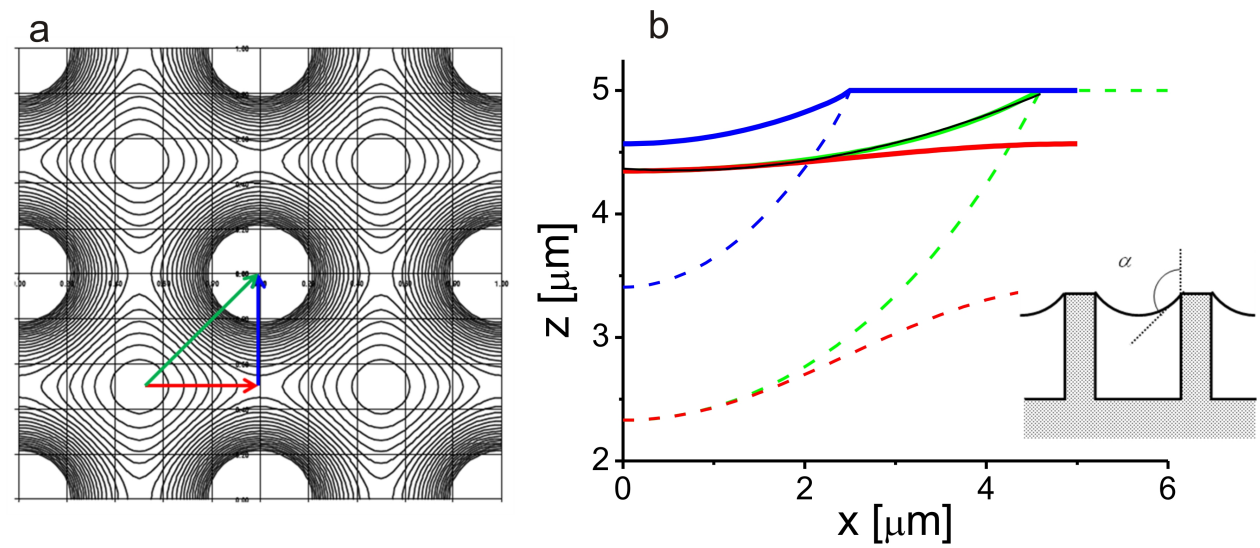

Figure 2.5: (a)-contour plot of $\zeta$ for $d=5 \mu \mathrm{m}$ numerically solved by Eq 2.2 (b)-numerical profiles corresponding to the experimentally observed maximum deflection (solid) and the profiles for the numerically obtained maximum deflection (dashed), respectively, along the 3-principal directions, which are designated in Fig.2.5(a).

pillar (implying contact line pinning) and, for symmetry reasons, $\partial w / \partial u=0$ for $u=0$ and $u=(a+d / 2) / R$ and similarly $\partial w / \partial v=0$ for $v=0$ and $v=$ $(a+d / 2) / R$. Note that the full problem depends via the boundary condition also on the dimensionless ratio $a / R$, which varies between 0.3 and 0.8 in the experiments. The numerical solution reproduces the characteristic shape of the air-water interface with its maxima of the deflection in the centers of the pits and the saddle points in between two adjacent pillars is given in Fig. 2.5. The contour-plot in Fig. 2.5(a) represents a complex three-dimensional deformation of the liquid-vapor interface. Fig. 2.5(b) shows the numerical profiles corresponding to the experimentally observed maximum deflection (solid) and the profiles for the numerically obtained maximum deflection (dashed), respectively, along the 3-principal directions, which are designated in Fig. 2.5(a). Here, $\alpha$ is determined from the diagonal profile (green), which also fits well with parabola shape (solid black). 


\section{ELECTRICAL SWITCHING OF WETTING STATES ON SUPERHYDROPHOBIC SURFACES}

\subsection{Results and disscussion}

According to the analysis described in previous section, the normalized deflection $\zeta_{0} / H$ should collapse if plotted versus the normalized parameter $\Lambda$, which is indeed the case (see Fig. 2.6. The solid lines in the panel of Fig. 2.6 show $\zeta_{0}$ vs $\Lambda$ for $d=10,8,5$ and $3 \mu \mathrm{m}$, respectively. While the model results and the experimental data agree very well for small $\Lambda$, we systematically underestimate the deflection for larger $\Lambda$. This is due to the parallel plate approximation in the calculation of the electric field, which neglects field enhancement effects due to the finite curvature of the interface (Additional finite element calculations performed for flat air-water interfaces indicated that deviations in the electric field can become of order $10 \%$, leading to $20 \%$ corrections to the Maxwell stress, in agreement with electrostatic calculations of the field experienced by a conductive sphere in front of a conductive plane). More importantly, however, the maximum deflection obtained numerically, the end point of the gray parts of the numerical results, which is beyond the scale of Fig. 2.6, is much larger than found experimentally.

What is the origin of this discrepancy and what determines the transition from the Cassie-Baxter to the Wenzel state? The numerical solutions become unstable as soon as the additional electrostatic energy gain for an incremental additional surface deflection outweighs the additional cost in interfacial energy. The corresponding interface deflections are more than twice the experimentally observed maximum deflections (see Fig. 2.6). Analyzing the numerical interface profiles reveals three important observations: (i) The angle between the air-water interface and the vertical side wall of the pillars is $\alpha \approx 150^{\circ}$ for the maximum $\Lambda$ yielding stable numerical profiles. This values is much larger than the advancing angle $\theta_{a d v}$ on the sidewall of the pillars. Hence, these solutions will not be observable experimentally due to depinning of the contact line. (ii) For the numerical profiles corresponding to the maximum deflection found in the experiments, we find the angle between the air-water interface and the vertical side wall of the pillars is close to $110^{\circ}$, in reasonable agreement with $\theta_{\text {adv }}$ (see Table 2.2). (iii) Similarly, extracting the critical angles from the maximum experimental deflection using the approximately parabolic surface shape (as justified by the numerical results; 
see Fig. 2.5 yields critical angles of the order $\alpha_{c} \approx 110^{0}$ (see Table 2.2). Hence, we conclude that the stability limit of the Cassie state is determined by the limiting angle w.r.t. the side wall, for which the microscopic three-phase contact lines remain pinned along the edges of the pillars. Upon exceeding this critical angle, the contact lines depin, move downward towards the flat bottom of the superhydrophobic surface and cause the transition to the Wenzel state. The stability limit is thus the same as in the case of a pressure-driven Cassie-to-Wenzel transition [55, 63. This result is not trivial. Exact calculations of the field distribution and surface deformation for a two-dimensional system show that the transition in that case can also be initiated by an instability of the air water interface in the center of a pit 64 indicating that the actual geometry of the interface can plays an important role.

\begin{tabular}{ccccc}
$d(\mu m)$ & $\theta^{*}$ & $\alpha_{c}$ & $U_{c}(V)$ & $U^{*}(V)(\beta=0.5)$ \\
\hline \hline 3 & $103^{0}$ & $110^{0}$ & 225 & 270 \\
5 & $109^{0}$ & $115^{0}$ & 175 & 177 \\
8 & $116^{0}$ & $108^{0}$ & 123 & 91 \\
10 & $120^{0}$ & $109^{0}$ & 105 & 0
\end{tabular}

Table 2.2: $\theta^{*}$ : critical angle for spontaneous propagation of the collapse, $\alpha_{c}$ : critical angle at the transition as obtained from the experiments, $U_{c}(V)$ : critical voltage at which transition occurs, and $U^{*}(V)(\beta=0.5)$ : estimated value for voltage at which the collapse will propagate

To understand why the transition nucleates along the apparent contact line, we note that the electric field between the drop and the substrate is not homogeneous in electrowetting. For flat substrates, sharp edge effects along the contact line give rise to a divergence of the electric fields in a region with a characteristic extension given by the thickness of the insulator [40]. For the present structured surfaces, the surface geometry and thus the field enhancement effects are more complex. Along the macroscopic contact line, there are sections of the microscopic contact lines with local contact angles of the same order as the macroscopic contact angle. Along these sections field enhancement leads to an increased Maxwell stress pulling the air-water interface downwards. Two-dimensional numerical estimates show a field enhancement up to $30 \%$, explaining the observed nucleation 


\section{ELECTRICAL SWITCHING OF WETTING STATES ON SUPERHYDROPHOBIC SURFACES}

along the apparent contact line.

Finally, we address the propagation of the Wenzel state across the drop-substrate interface. In order to propagate from one filled pit to the next, the liquid has to pass the gap between two adjacent pillars, which has the width $d$. The energy gain upon advancing by a distance $\delta x$ within this gap is $\delta G=\delta x[(2 h+d)(\sigma-$ $\left.\left.\sigma_{s l}\right)+d\left(\sigma-\left(C_{C}-C_{W}\right) U^{2} / 2\right)\right]$, where $C_{C}$ and $C_{W}$ are the local capacitances in the Cassie and in the Wenzel state, respectively. Analogous to the situation without electric field [65], propagation is expected if $\delta G>0$. Writing the (positive) differential capacitance as $C_{C}-C_{W}=\beta \varepsilon_{0}\left(\varepsilon_{d} / h_{d}-\varepsilon / H\right)=\beta \varepsilon_{0} \varepsilon h / H h_{d}$, where $\beta$ is a correction factor of order unity accounting for the exact field distribution, this criterion yields a geometry-dependent critical voltage $U^{*}$ above which propagation of the Wenzel state is energetically favorable:

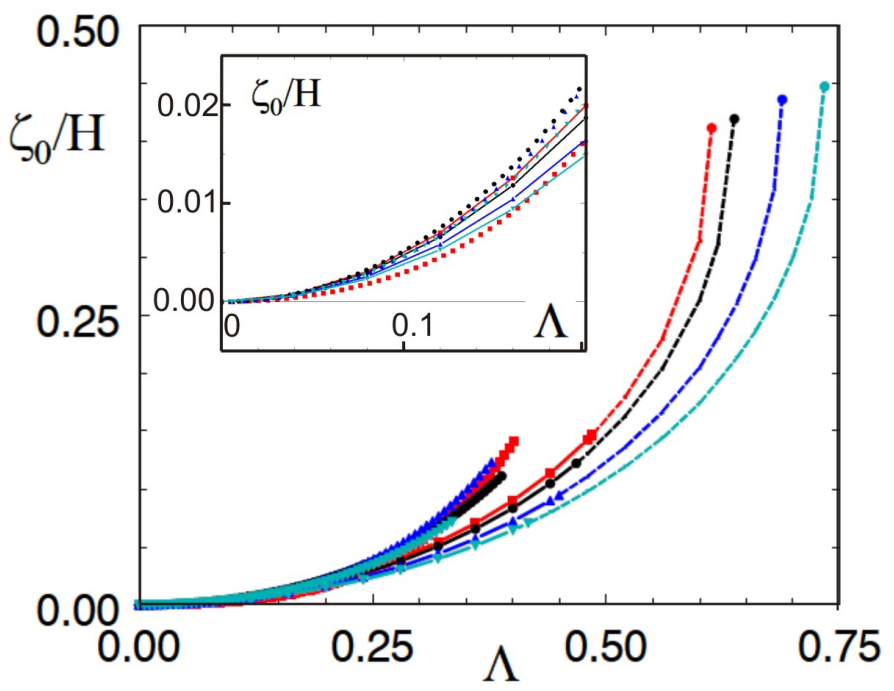

Figure 2.6: Normalized deflection versus normalized electrostatic force . Symbols: experimental data for variable post spacing $d=10 \mu m$ (red) squares], 8 (black circles), 5 [(blue) up triangles], and $3 \mu m$ [(green) down triangles]. Symbols with lines: numerical calculations. Inset: Zoomed view of main panel.

$$
U^{*}=U_{0} \sqrt{\frac{\varepsilon h_{d}}{\beta \varepsilon_{d} h}\left(\frac{\cos \theta_{Y}}{\cos \theta^{*}}-1\right)}
$$

Here represents the critical angle, for which spontaneous propagation at zero 
voltage occurs [65] and is the characteristic voltage related to the electrowetting number. Propagation of the Wenzel state is expected if for a given sample. For this condition is indeed fulfilled for two largest spacings whereas it is not fulfilled for the smallest spacing (see Table 2.2), confirming that the model correctly captures the physical effects controlling propagation of the Wenzel state.

\subsection{Conclusions}

The results described here demonstrate the flexibility and the control of superhydrophobicity that can be achieved using electric fields. At low voltages, the reversible bending of the micromenisci allows for detailed tuning of both hydrodynamic slip [66] and of optical diffraction from such surfaces [55]. For the irreversible transition to the Wenzel state, we clearly identified that depinning of the contact lines determines the critical voltage. Combined with the criterion for the co-existence of the Cassie state and the Wenzel state, we are confident that this principle will enable the design of novel surface and electrode geometries, allowing for local and probably even reversible switching between the two competing wetting morphologies. 
2. ELECTRICAL SWITCHING OF WETTING STATES ON SUPERHYDROPHOBIC SURFACES 


\section{Chapter 3}

\section{Electrically induced reversible transitions on superhydrophobic surfaces}

\subsection{Abstract}

In this chapter, we present two approaches to achieve electrowetting induced reversible transitions from the Cassie-Baxter to the Wenzel states on superhydrophobic surfaces. We will show that oscllations of three-phase contact line under low frequenciy electric field, is able to initiate a transition from the partial Wenzel to the Cassie-Baxter state. We also will demonstrate reversible transitions using patterned electrodes ${ }^{1}$

${ }^{1}$ Part of this chapter has been published in "Electrical Switching of Wetting States on Superhydrophobic Surfaces: A Route Towards Reversible Cassie-to-Wenzel Transitions", G. Manukyan, J. M. Oh, D. van den Ende, R. G. H. Lammertink, and F. Mugele [49]. 


\section{ELECTRICALLY INDUCED REVERSIBLE TRANSITIONS ON SUPERHYDROPHOBIC SURFACES}

\subsection{Introduction}

An important area of application of superhydrophobic surfaces is reversible superhydrophobicity. That is the ability of a surface to switch between the hydrophobic and hydrophilic properties under the influence of the, light irradiation (ultraviolet or visible), or temperature, etc. [22, 23, 25, 25, 26, 27, 28, 29, 30, 31, 32, 34] . Howewer, in many of mentioned works only the properties of the surface that are switched from superhydrophobic to not superhydrophobic. It is more important the ability to switch a given drop from one state to another. This area of research has emerged since 2004, and a number of important findings have been made, including the ability to switch between the Cassie-Baxter and Wenzel states by using electrowetting effect [35]. In 2007 Krupenkin et al. [33] reported that droplet behavior can be reversibly switched between the Cassie-Baxter state and the Wenzel state. They demonstrated the first solution for the reversible wetting on such surfaces. A very short electrical current impulse applied to the substrate leads to surface heating. The temperature reaches $240^{\circ} \mathrm{C}$, causing liquid boiling and droplet expelling from the surface. Even though this technique is easy to implement, it is hard to imagine such an integrated system within a Lab-on-Chip. For biological applications the heating would cause significant damage within the drop. Moreover, this expulsion creates satellite droplets.

The key factors for achieving reversible transitions from the Cassie-Baxter to the Wenzel states are: (i) thermodynamically favorable Cassie-Baxter state for the droplet, and (ii) presence of entrapped air in Wenzel state. The second factor may be achieved in two ways: via using double-scale (micro/nano) roughness [12], at which even if at micro scale the droplet will be in the Wenzel state, there will be air entrapped in nanoscale, or by controlled partial transitions to the Wenzel state. In both cases the drop-substrate interface remains vapor filled, such that the reverse switch only requires the motion of contact lines but not the nucleation of the vapor "from scratch."

In this chapter we demonstrate two approaches of electrowetting induced reversible transitions from the Cassie-Baxter to the partial Wenzel state. In our firs approach we attempt to use electrowetting induced vibrational energy of the oscillations of a droplet at metastable Wenzel state to switch it to the Cassie 
Baxter state. The second approach is to use patterned electrodes under the microstructure. This allows us to transform the surface only locally into the Wenzel state above activated patterned electrodes

\subsection{Wenzel to Cassie-Baxter transitions using low frequency oscillations}

The first approach is an electrical analogous of mechanical vibration induced Wenzel to Cassie-Baxter transition [24]. In their experiments Boreyko et al. used mechanical vibrations to overcome the energy barrier for the transition from the sticky Wenzel state to the non-sticking Cassie-Baxter state. In case of ac electrowetting at low-frequency range, they observed shape oscillation of a droplet in [67, 68]. This oscillation results from the time-varying electrical force concentrated on the three-phase contact line. In this study we attempt to use electrowetting induced vibrational energy of the oscillations of a droplet at metastable Wenzel state to switch it to the Cassie-Baxter state instead of mechanical vibration of the substrate described in [24].

\subsubsection{Materials and methods}

The samples for this experiment were prepared in similar manner as for the experiments described in chapter 1. Superhydrophobic surfaces were prepared using photolithography technique: ITO covered glass was spin-coated with $5 \mu \mathrm{m}$ thich SU-8 3005 photoresist, then exposed at UV light and developed. This provides an homogenous layer of a dielectric (thickness $d=5 \mu \mathrm{m}$; dielectric constant $\varepsilon=3.2$ ), which prevents the samples from breakdown when under electric field the microcavities are field with water. On top of that homogeneous layer the microstructuring was performed, square lattices of round pillars (diameter $2 a=5 \mu \mathrm{m}$ ; height $h=5 \mu m$ distance between the pillars $3 \mu \mathrm{m}$ ) The corresponding values of Wenzel's surface roughness factor $r$ and fractional area of the pillars $f$ are: $r=2.23$, and $f=0.307\left(r=A_{\text {real }} / A A_{\text {real }}\right.$ is true solid surface area following the topography; $A$ is projected surface area). The Wenzel state is thermodynamically not favorable for these parameters. The surfaces are hydrophobized by 


\section{ELECTRICALLY INDUCED REVERSIBLE TRANSITIONS ON SUPERHYDROPHOBIC SURFACES}

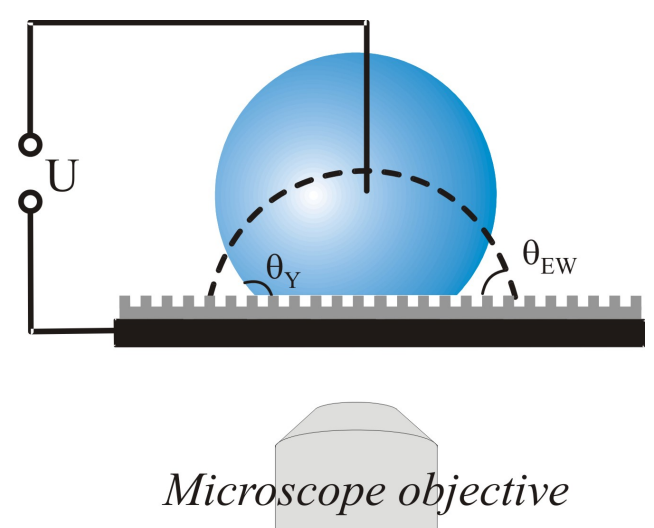

Figure 3.1: Electrowetting setup: a voltage $U$ is applied between a droplet and an electrode covered with a dielectric micro-patterned surface. The contact area is monitored with an inverted microscope from the bottom through the superhydrophobic substrate.

dip-coating in a $0.01 \%$ Teflon AF (Dupont) solution. The contact angle on the flat surface area next to the pillar array of $\theta_{Y} \approx 115^{0}-120^{0}$.

The macroscopic drop shape was controlled by applying a voltage (here: ac) between the drop via an immersed metal wire and the ITO electrode. In this case the metal wire was chosen to be thicker than in previous experiments to prevent the droplet from detaching during the oscillations. The drop-substrate interface wass illuminated with monochromatic light $(\lambda=510 \mathrm{~nm})$ from below and observed in reflection mode using an inverted microscope 3.1 .

\subsubsection{Observations}

The transition from Cassie-Baxter to Wenzel state was achieved in a similar way as in chapter 1 . Here for convenience we used $1 k H z$ ac voltage instead of dc. To obtain a partial transition to the Wenzel state $\sim 300 \mathrm{~V}$ was applied (see Fig. 3.2 a). Dark dots at the inner side of the three phase contact line indicate that these pits have been filled with water. After removing the voltage, as expected, the droplet remains in partial Wenzel state. Afterwards the applied voltage was set to $(\sim 150 \mathrm{~V})$ and the frequency to $64 \mathrm{~Hz}$. This voltage is not enough for Cassie-Baxter to Wenzel transition, thereby at low frequencies the three phase 
contact line oscillations presumably may only empty filled air-pockets, bringing the droplet to Cassie-Baxter state. Indeed, after low frequency was applied, the three phase contact line starts to oscillate emptying filled air-pockets. Circles in Fig. $3.2 \mathrm{~b}$ and $\mathrm{c}$ highlight the area where there are still some air-pockets filled after 3 and $6 s$ of oscillations respectively. Further oscillations result in complete transition to the Cassie-Baxter state (see Fig. 3.2 d-f).
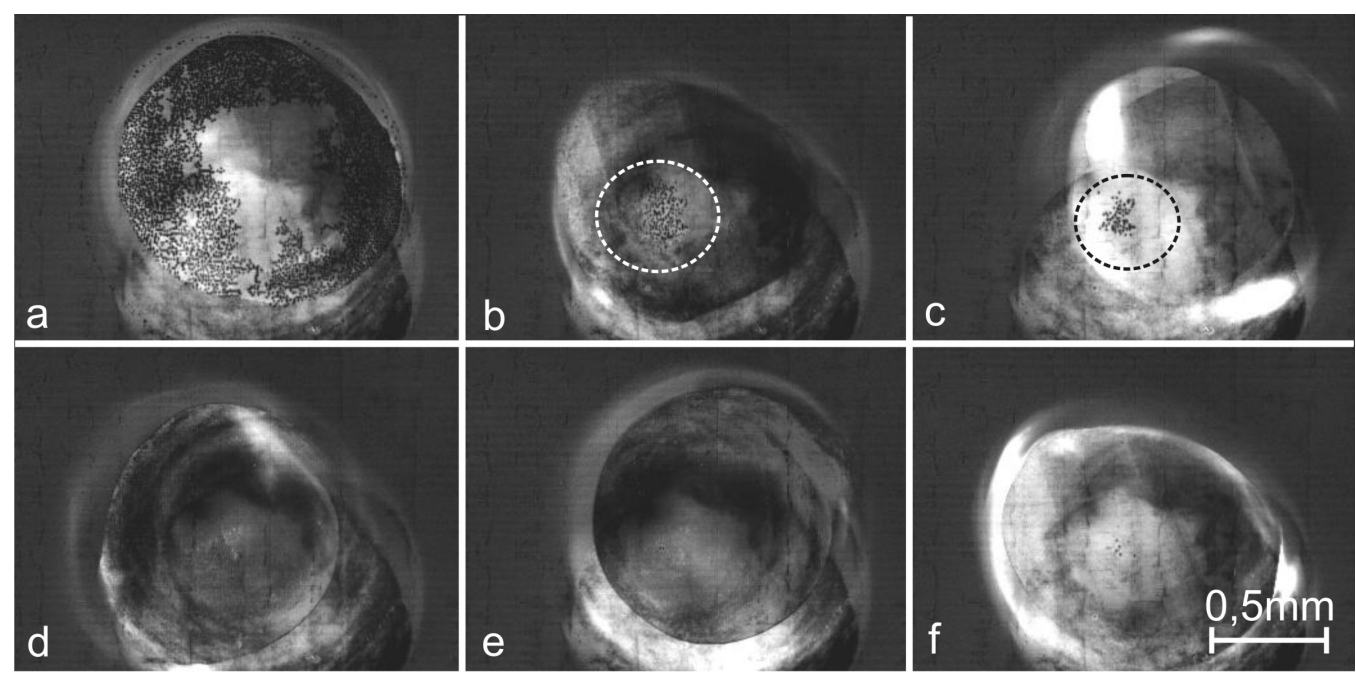

Figure 3.2: Snapshots of droplet contact area: (a) in the partial Wenzel state, (b), (c) the droplet is still in partial Wenzel state (d)-(f) droplet is oscillating around metal electrode being in mobile Cassie-Baxter state.

\subsection{Reversible transitions using patterned elec- trods}

The second approach is to use patterned electrodes under the microstructure. This allows us to transform the surface only locally into the Wenzel state above activated patterned electrodes, while other regions of the drop-substrate interface remain vapor filled, such that the reverse switch only requires the motion of contact lines but not the nucleation of vapor "from scratch." 


\section{ELECTRICALLY INDUCED REVERSIBLE TRANSITIONS ON SUPERHYDROPHOBIC SURFACES}

\subsubsection{Materials and methods}

For these experiments, surfaces with $8 \mu m$ wide parallel rectangular grooves was used (see Fig. 3.3). The sample preparation process is the same as for previous experiments, with the difference that in this case we etch away a part of ITO layer leaving a single stripe (Fig. 3.3 a) or an array of $8 \mu \mathrm{m}$ wide ITO stripes perpendicular to the direction of the grooves (Fig. $3.3 \mathrm{~b}$ ). The distance between ITO stripes was $50 \mu \mathrm{m}$. In addition, the sample was cleaned using plasma treatment, in order to improve the quality of Teflon coating. In these experiments we used $1 k H z$ ac voltage. As in all previous experiments, here also we look at the droplet contact area.
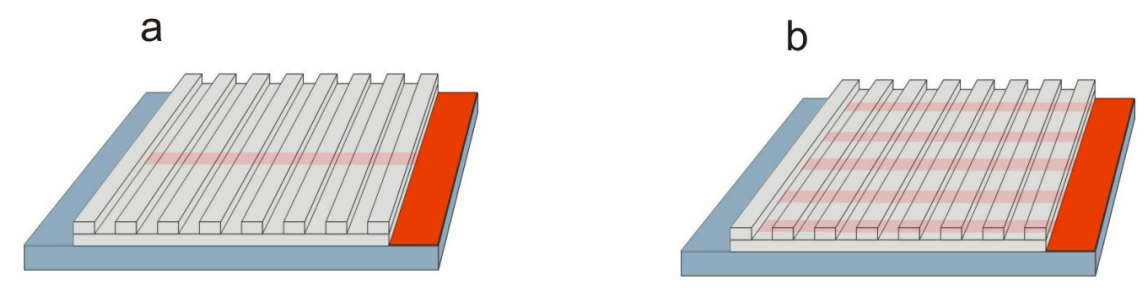

Figure 3.3: Sketch of a sample with patterned electrodes : (a) single-stripe electrode geometry $10 \mu \mathrm{m}$ wide electrode (red stripe) is perpendicular to grooves. (b) Patterned electrode geometry

\subsubsection{Observations}

Let us first discuss single stripe electrode configuration. After applying a voltage between the droplet and the electrode $(\sim 400 \mathrm{~V})$, the liquid interface above the electrodes turns dark, indicating filling of the microchannel (see Fig. ??b). The fact that only above electrode/channel intersection (and small vicinity around it) we observe variation of the reflected light suggests that everywhere except the intersection the liquid interface remains pinned to the top of the channel. The interface detaches from the top of the channel only above the electrode. When 


\subsection{Reversible transitions using patterned electrods}

the voltage is turned off the droplet returns to the initial Cassie-Baxter state( Fig. ??a). Thus, with this system we achieve reversible transitions between the Cassie-Baxter and the Wenzel states. The crucial factor enabling reversible transitions in such a system is the small hysteresis of the surface. The Laplace pressure which tends to empty the michrochannel should be able to overcome the contact line pinning.

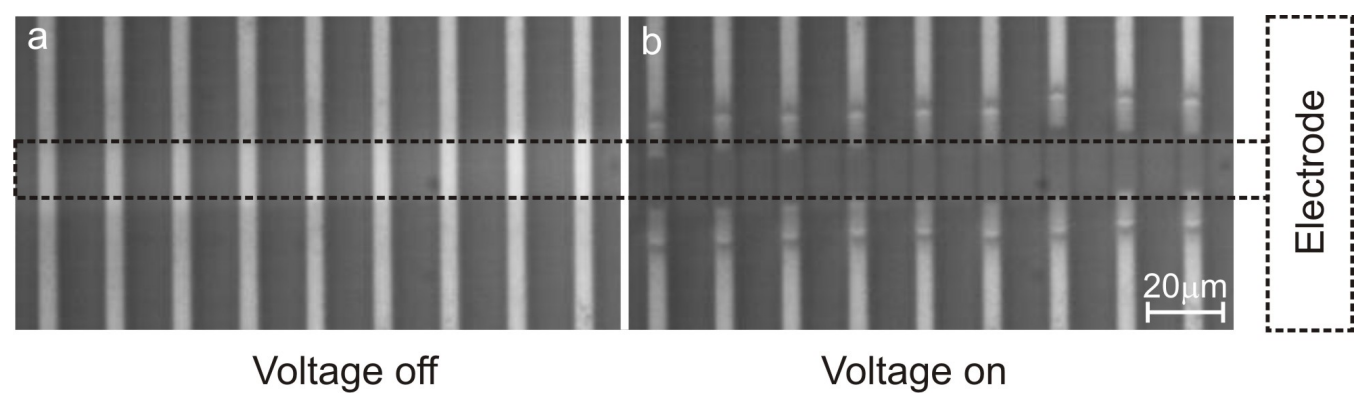

Figure 3.4: Snapshots of a droplet contact area for the single stripe electrode geometry: the electrode (dashed rectangle) is perpendicular to the direction of grooves. (a) at $400 \mathrm{~V}$ the droplet is in the partial Wenzel state, (b) after turning off the voltage it returns to the initial Cassie-Baxter state.

Hence we observed reversible transitions with single stripe electrode, let us now try the same experiment with an array of electrodes perpendicular to the direction of the grooves (Fig. 3.3 b). In this case, the applied voltage causes only partial transition from the Cassie-Baxter and Wenzel state, since the electrostatic Maxwell stress mainly acts to the parts of liquid/gas interface above electrode/channel intersection, bending them downwards. However, as can be seen from the Fig. 3.5a dark patches appear above not every electrode/channel intersection, more frequently we see transition on every second intersection.

In order to qualitatively understand this effect let us consider 3 consecutive intersections on the same channel as illustrated in Fig. 3.6. When the intersections 1 and 3 are in Wenzel state, the air pressure inside the channel between these electrodes increases $\sim 20 \%$, (the volume decreases). This additional pressure is in the same order of magnitude as the Laplace pressure, which adds up to the Laplace pressure preventing the transition at the intersection 2. 


\section{ELECTRICALLY INDUCED REVERSIBLE TRANSITIONS ON SUPERHYDROPHOBIC SURFACES}

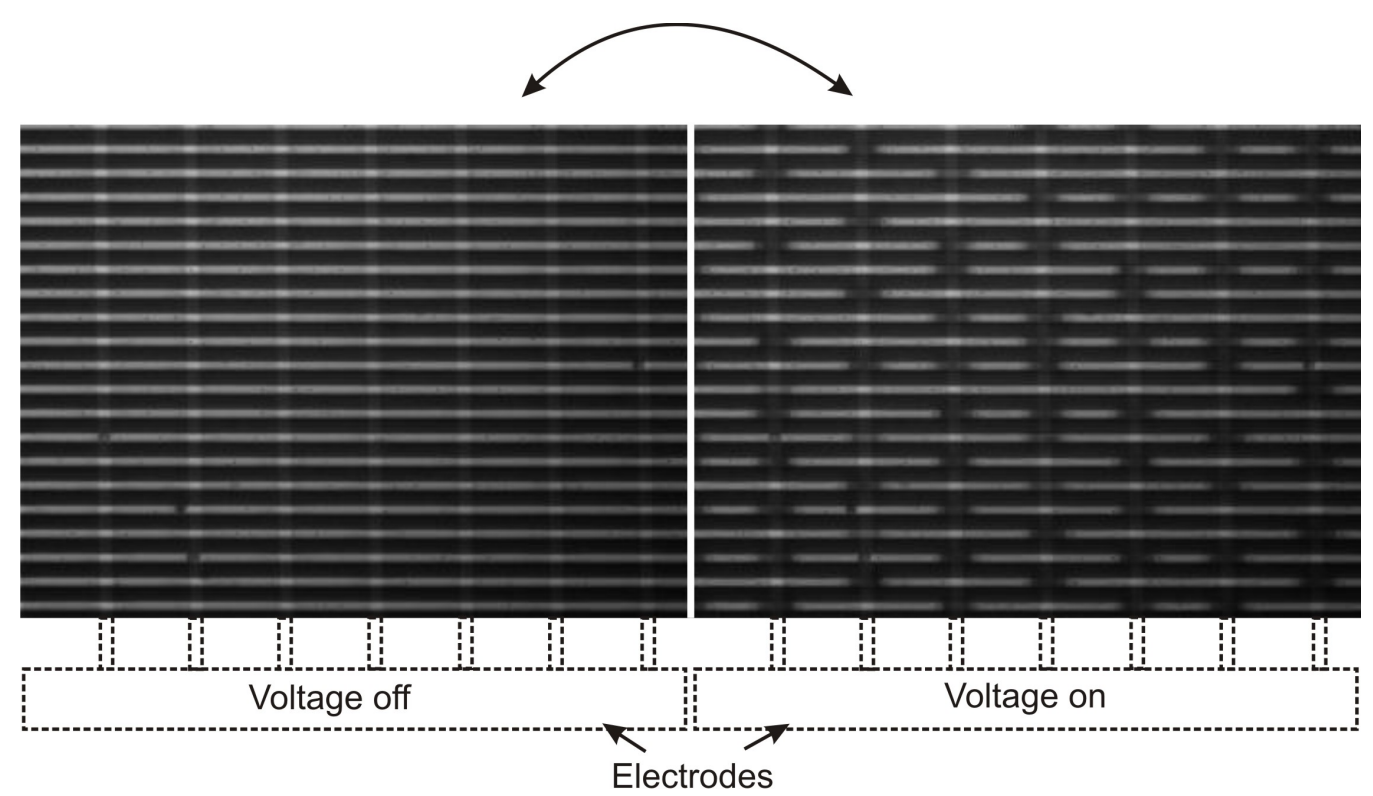

Figure 3.5: Snapshots of a droplet contact area: patterned electrodes (vertical stripes) are perpendicular to the direction of grooves. (a) at $400 \mathrm{~V}$ the droplet is in the partial Wenzel state, (b) after turning off the voltage it returns to the initial Cassie-Baxter state.

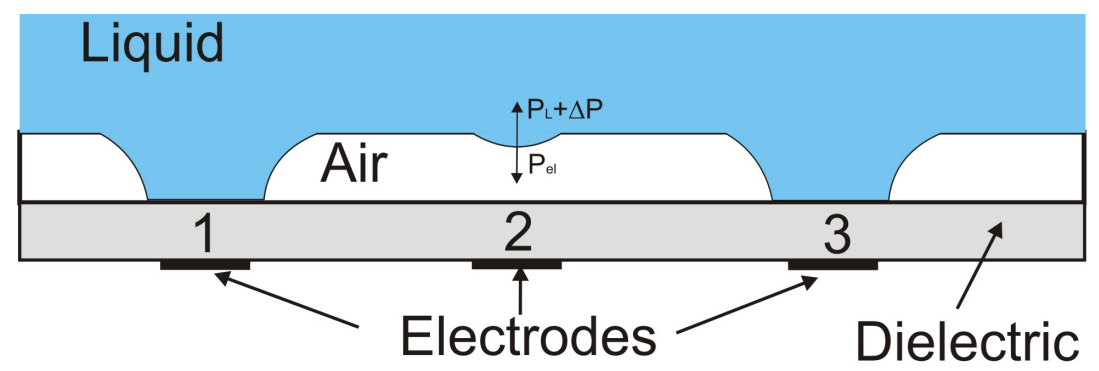

Figure 3.6: Sketch of the cross section of the system along the direction of electrodes: the droplet is in the partial Wenzel state on a patterned electrode system.

Described approach for reversible transition between the Cassie-Baxter and the Wenzel states deserves an attention, since it is easy to implement in microfluidic systems. However more research is required to determine the optimal set of parameters such as width of the electrodes, distance between electrodes, etc. which will provide better performance of the system. Unfortunately the study of the these parameters is out of the scope of this work. 


\subsection{Conclusions}

In this chapter we present two approaches to achieve electrowetting induced reversible transitions between Cassie-Baxter and Wenzel states. For both approaches reversible transition between wetting morphologyes was demonstrated. The shape oscillations of the droplet under low frequency ac electric field $(<$ $100 \mathrm{~Hz}$ ) are able to switch the droplet from the partial Wenzel to the CassieBaxter state. However, the time required for the transition is rather long $(<10 \mathrm{~s})$.

The second approach was the usage of patterned electrodes, which allows to obtain controlled transition to the partial Wenzel state, leaving air bubbles entrapped between electrode/microchannel intersections. In this case the transition time from the Wenzel to the Cassie-Baxter state is $\sim 5-10 \mathrm{~ms}$. 
3. ELECTRICALLY INDUCED REVERSIBLE TRANSITIONS ON SUPERHYDROPHOBIC SURFACES 


\section{Chapter 4}

\section{Electric field driven instabilities on superhydrophobic surfaces}

\section{1 abstract}

In this chapter we study possible mechanisms for the transition from the Cassie state to the Wenzel state on superhydrophobic surfaces under the influence of electric fields as a function of the aspect ratio and the wettability of the surface, both numerically and experimentally. Fully self-consistent calculations of both electric field distribution and surface profiles show that this instability evolves from a global one towards a local Taylor cone-like instability for increasing aspect ratio of the cavities, which were confirmed with our experimental results 1

\footnotetext{
${ }^{1}$ Modeling part of this chapter has been published in "Electric-field-driven instabilities on superhydrophobic surfaces", Oh, J. M., Manukyan, G., Ende, D. van den and Mugele, F. 64]., The experimental part to be published
} 


\section{ELECTRIC FIELD DRIVEN INSTABILITIES ON SUPERHYDROPHOBIC SURFACES}

\subsection{Introduction}

When a droplet is placed on a textured hydrophobic surface, air remains between the droplet and the substrate leading to a composite liquid-solid-air interface containing a large number of microsopic menisci [50]. The resulting reduction of the liquid-solid interfacial area is at the origin of the large contact angle and all other favorable properties of superhydrophobic surfaces, such as reduced frictional drag and high optical reflectivity. Yet this superhydrophobic Cassie state is not the only wetting state on textured hydrophobic surfaces. Rather, it competes with the Wenzel state, in which liquid fills all the cavities on the rough surface. Due to the particularly high solid-liquid interfacial area in this case, many properties of the Cassie state turn into their reverse. In order to optimize the stability of the superhydrophobic state, a substantial amount of work has been devoted to understanding the physical mechanisms controlling the transition from the Cassie to the Wenzel state [63, 65, 69, 70, 71, 72, 73].

In chapter 1 we presented a model based on the local balance of electrostatic forces and the capillary pressure to explain the deformation of the micromenisci at the drop-substrate interface and their collapse, which initiates the transition to the Wenzel state at some critical voltage. For the conditions of those experiments, it turned out that the stability limit of the Cassie state was determined by depinning of the contact line, similar to the pressure-driven collapse of conventional superhydrophobic surfaces [63, 73]. However, these experiments also demonstrated that the geometry of both the surface texture and the electrodes has an important impact on the nature and in particular on the reversibility of the transition.

In this chapter we focus on transition scenarios from the Cassie to the Wenzel state in a simple generic geometry, namely a single cylindrically symmetric cavity with a homogeneous electrode at the bottom and a perfectly conductive liquid at the top (see Fig. 4.1). Using self-consistent numerical calculations we determine the full equilibrium distribution of the electric field and the corresponding surface profiles based on the local balance of the Maxwell stress and the Laplace pressure at the liquid-vapor interface. We validate experimentally the numerical results, in particular we show experimentally that under influence of an electric field, the 
liquid interface is no longer spherical. We also demonstrate two scenarios for the Cassie-Wenzel transition under applied electric field.

\subsection{The model}

We consider a flat electrode at the bottom and a second electrode with a round hole of radius $a$ at a distance $h$, as shown in Fig. 4.1. Liquid fills the space above the top electrode and forms a deformable meniscus in the hole. The threephase contact line is pinned along the edge of the hole. For the purpose of our calculation, the space in between the two electrodes is filled with air.

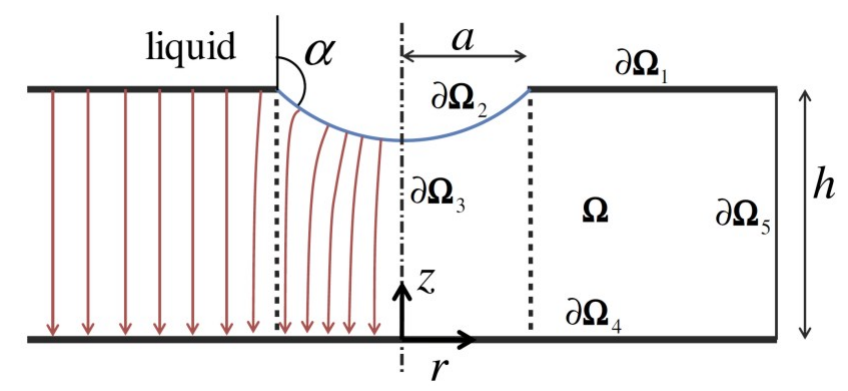

Figure 4.1: System description: interface deflection in axisymmetric cavity.

In the absence of free charge, the distribution of the electric potential $\phi$ is governed by the Laplace equation within the computational domain $\Omega$.

$$
\nabla^{2} \phi=0 \quad \text { in } \quad \Omega
$$

We use boundary conditions of constant potential on the two electrodes and the the liquid and vanishing radial fields on the symmetry axis and at the outer edge of the domain for $r \gg a$, (see also Fig. 4.1):

$$
\begin{array}{rlrlrl}
\phi & =U & \text { on } & & \partial \Omega_{1} \text { and } \partial \Omega_{2}, \\
\frac{\partial \phi}{\partial r}=0 & \text { on } & & \partial \Omega_{3} \text { and } \partial \Omega_{5}, \\
\phi=0 & \text { on } & & \partial \Omega_{4} .
\end{array}
$$




\section{ELECTRIC FIELD DRIVEN INSTABILITIES ON SUPERHYDROPHOBIC SURFACES}

In addition, the normal stress condition at the liquid-air interface, which represents the local balance between the Laplace pressure and the Maxwell stress, is imposed on the free surface $\left(\partial \Omega_{2}\right)$ to determine the shape of the interface.

$$
\gamma(\boldsymbol{\nabla} \cdot \boldsymbol{n})=\left[\left[\boldsymbol{n} \cdot \boldsymbol{T}^{e} \cdot \boldsymbol{n}\right]\right]
$$

where $[[\cdot]]=(\cdot)_{\text {out }}-(\cdot)_{\text {in }}, \gamma$ is the surface tension of liquid-air interface, $\boldsymbol{n}$ the unit normal vector on the interface, and $\boldsymbol{T}^{e}$ the Maxwell stress, respectively. The Maxwell stress is defined by $\boldsymbol{T}^{e}=\varepsilon \varepsilon_{0}\left[\boldsymbol{E} \boldsymbol{E}-\frac{1}{2} E_{n}^{2} \boldsymbol{I}\right]$, where $\varepsilon$ is the dielectric constant, $\boldsymbol{E}$ the electric field, and $E_{n}$ the absolute value of $\boldsymbol{E}$, respectively. The curvature $(\kappa)$ of the surface is given by the divergence of the normal vector.

$$
\kappa=\nabla \cdot n
$$

Eq. 4.1-4.3 define a free boundary problem, which we numerically implement using the commercial software package COMSOL Multiphysics. The Moving Mesh application in COMSOL is applied to capture the free boundary (for more details of the model see [64]).

\subsection{Materials and methods}

The experimental setup consists of a flat ITO-covered glass plate coated with $5 \mu \mathrm{m}$ SU-8 covered electrode at the bottom, and $130 \mu \mathrm{m}$ thick microscope slide with a round hole of radius $a(2 a=1.2 \mathrm{~mm})$ at the top (see Fig. 4.2 a). The distance between plates $h$ (aspect ratio $H=h / a$ ) is the main parameter in our experiments. Water fills the space above the microscope slide and forms a deformable meniscus in the hole. White dashed lines in Figs. $4.2 \mathrm{~b}$ and cindicate the lower surface of the top plate. Our area of interest lies below the dashed line.

Naturally, the sessile drop at the hole forms a meniscus with a finite radius of curvature due to Laplace pressure (see Fig. 4.2 b). The black segment below the dashed line in the Fig. $4.2 \mathrm{~b}$ is the cross-section of the water meniscus (the segment above the dashed line is the reflection of it on the top plate). To eliminate the initial curvature of the meniscus, the drop was connected to a hydrostatic head, in which the liquid level was adjusted to provide a well defined pressure equal to 

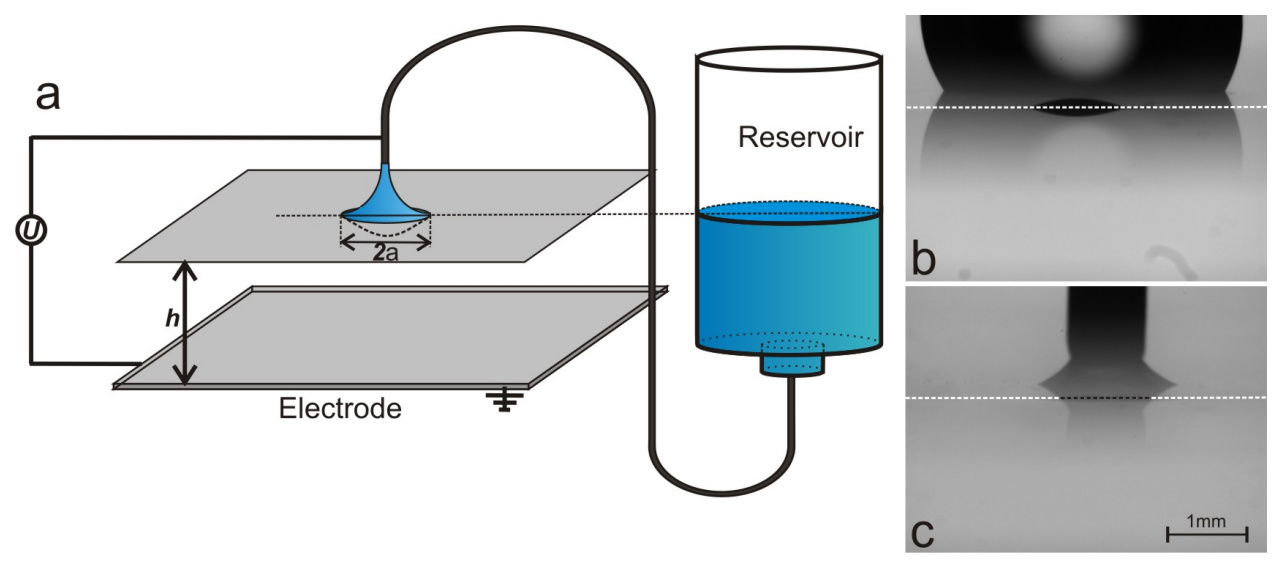

Figure 4.2: (a) Experimental setup: Drop of water, which is connected to water reservoir, is placed on a microscope slide containing a round hole. (b) Snapshot of a sessile drop on a hole (c) Snapshot of a drop connected to a hydrostatic head.

ambient pressure. As a result we obtain a flat initial meniscus. The black dashed line in Fig. 4.2. indicates the lower "edge" of the hole with a flat meniscus.

An electric field was applied between the drop and the bottom electrode and snapshots of a side-view of the liquid interface were taken upon increase of applied voltage (camera placed parallel to the electrodes). Fig. 4.3 shows typical sideview images of the liquid interface under influence of electric field. From these images the maximal deflection $\zeta$ of the meniscus as well as exact profiles of the liquid interface were extracted for series distances between plates $H$ and applied voltages $V$.

\subsection{Results and discussion}

As discussed in chapter 1, the stability of the liquid interface under applied electric field is governed by local balance between electrostatic Maxwell stress, pulling outwards along the surface normal, and Laplace pressure of the curved interface. This equilibrium holds for voltages smaller than some critical value $\left(V<V_{c}\right)$, after which the Laplace pressure cannot balance the Maxwell stress anymore and the system becomes unstable. 


\section{ELECTRIC FIELD DRIVEN INSTABILITIES ON SUPERHYDROPHOBIC SURFACES}

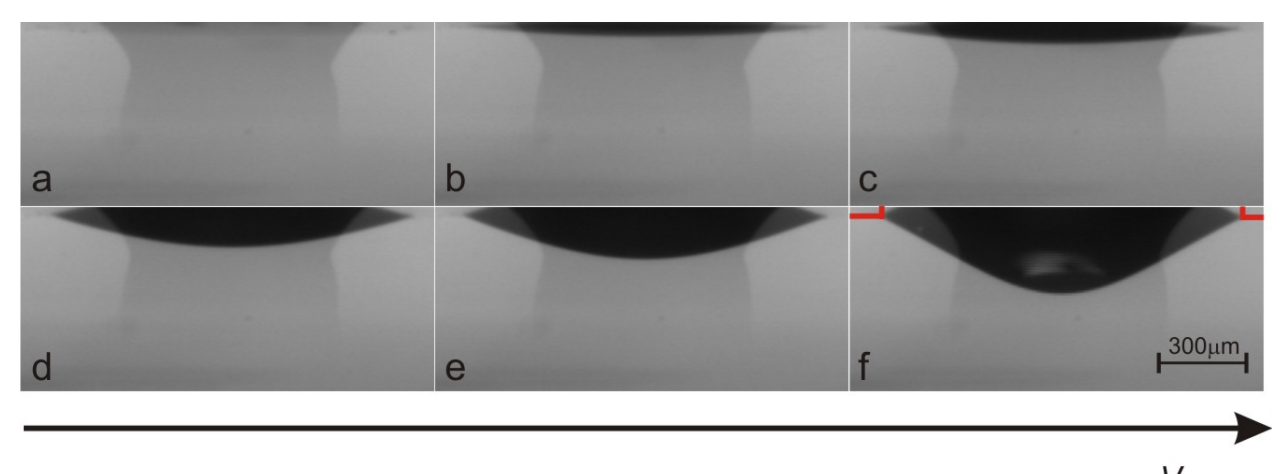

V

Figure 4.3: Side-view images of water meniscus at different values of applied electric field $(H=0,64)$

Let us first discuss the behavior of the liquid interface for voltages below the critical value focusing on the shape of the interface. Fig. 4.3 shows series of side-view images of the liquid interface under influence of electric field (the voltage increases from left to right). Upon increasing the voltage the bending increases gradually up to a critical maximum value $V_{c}$ after which the system becomes unstable. For voltages closer to $V_{c}$ one can notice that the liquid interface is rather conical than spherical, (see Fig. 4.3 ). This is due to the fact that the distribution of the electric force is not homogenous along the liquid-gas interface, which leads to non-spherical deformation of the interface. Equilibrium surface profiles obtained by calculating the electric field distribution and the surface profile by solving Eqs. (4.1)-(4.3) also confirm nonspherical nature of liquid interfaces [64]. To characterize the shapes of the liquid interfaces under electric field we compare the experimentally observed liquid interfaces to ones obtained from numerical simulations (Fig. 4.5). The dashed lines in the figure correspond to numerical results, whereas full colored lines to experimental data. There is no fitting parameter introduced to match numerical and experimental results. The voltage difference between corresponding numerical results and the experiment does not exceed $\pm 10 \mathrm{~V}$, which is below the limit of experimental error. The comparison proves consistency of the model, and provides an experimental evidence of non-spherical deformation of a liquid interface under applied electric field.

We can also determine the maximal deflection of the liquid interface as a 


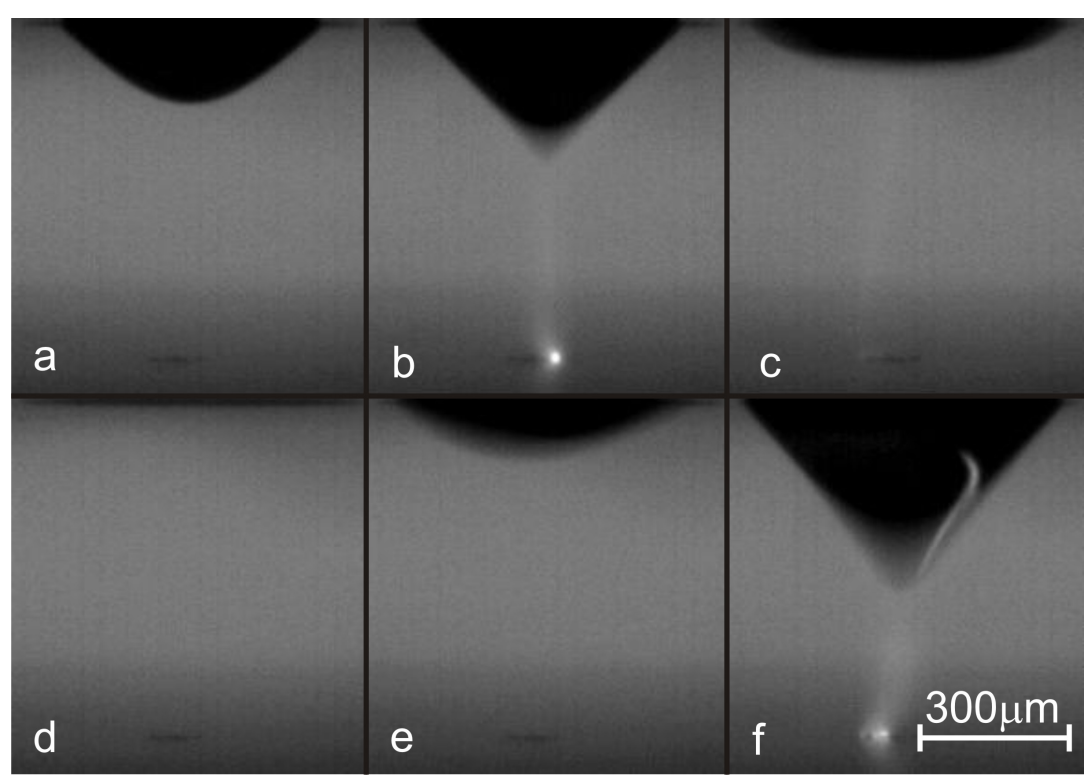

Figure 4.4: Snapshots of oscillating meniscus: the frames are taken $50 \mathrm{~ms}$ after each other.

function of applied voltage. Fig. 4.6 presents comparison of experimentally and numerically obtained deflection of the liquid interface for various values of aspect ratio $H$. Here dashed lines correspond to numerical results, symbols to the experimental data. The error bar represents averaging of maximal deflection for a given voltage in 3 runs of the experiment.

Let us now discuss the the situation when the applied votage exceeds the critical value. When $V>V_{c}$, the Laplace pressure cannot balance the Maxwell stress anymore, and thus, the system becomes unstable. For different values of $H$ the instability of the liquid interface manifests itself in a different manner: For $H<H_{c}\left(H_{c} \approx 0,4\right)$ increasing of applied voltage beyond $V_{c}$ leads to the meniscus touching the bottom of the cavity. After removing the applied voltage, the liquid interface remains pinned to bottom electrode due to large hysteresis, which apparently, the Laplace pressure is not able to overcome. This scenario displays similarities with the 'sagging' scenario for the pressure-driven transition on conventional superhydrophobic surfaces [63, 171. Yet, the sudden jump from a substantial finite distance is a characteristic of the electrically driven interface 


\section{ELECTRIC FIELD DRIVEN INSTABILITIES ON SUPERHYDROPHOBIC SURFACES}

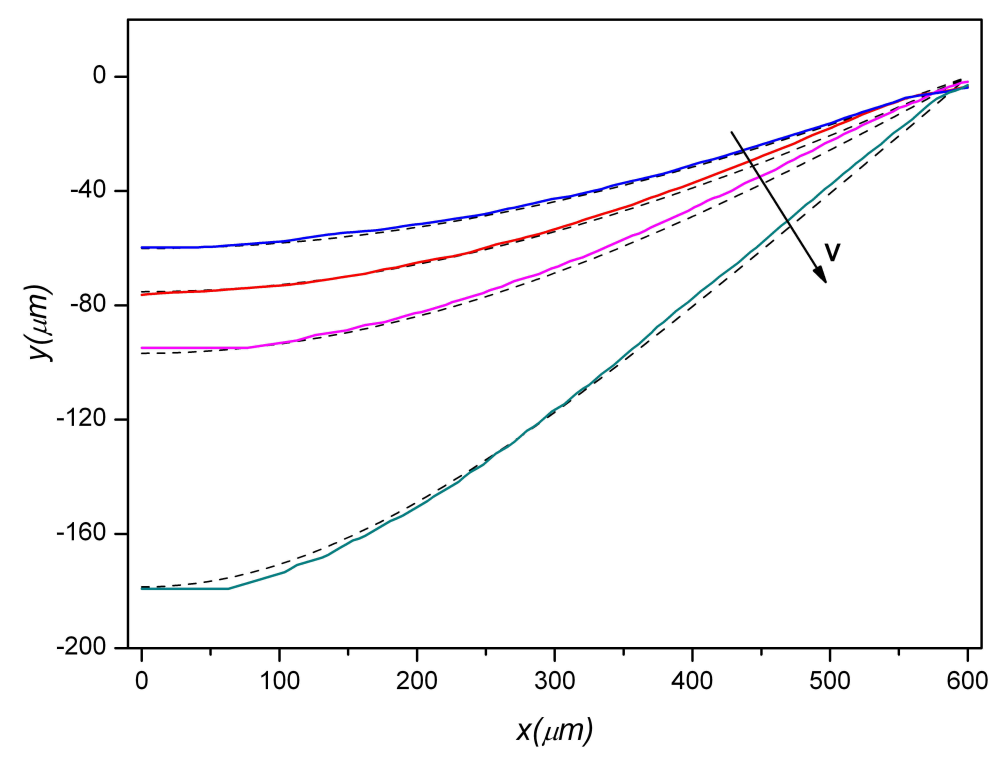

Figure 4.5: Liquid surface profiles obtained from numerical simulation and experiment. Dashed lines correspond to numerical results, solid lines experimental data for 1400, 15001600 and $1700 V(H=0,83)$

instability. For conventional superhydrophobic surfaces such jumps should also exist, but only on the length scale of the scale of the van der Waals (or some other molecular) attraction, i.e. on the scale of a few nanometers.

The situation is different for $H>H_{c}$. When the applied voltage reaches the critical value $V_{c}$ the sharp tip of the meniscus starts to oscillate, at the top of each oscillation ejecting a narrow jet of presumably charged microdroplets. Fig. 4.4 shows a typical snapshots of an unstable meniscus. The frequency of the oscillation depends on applyed voltage: more we increase the voltage beyond $V_{c}$ faster the meniscus oscillates. This phenomenon reminds a classical Taylor cone instability and electrospraying [74, 75]. The half opening angle of the cone at the jet formation in our experiments was $\approx 47-50^{\circ}$ (Taylor observed in his experiments a half opening angle of $\left.49,3^{0}\right)$.

For $H>>1$, the distribution of the electric field in the vicinity of the meniscus is no longer affected by the presence of the counter electrode at the bottom. This 


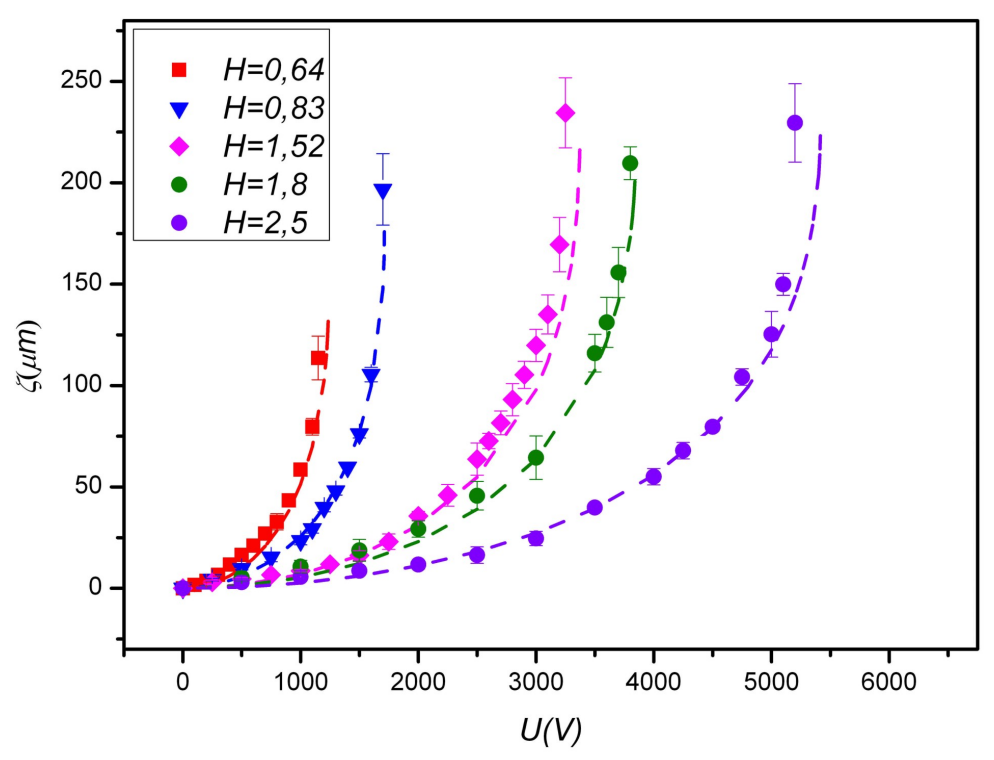

Figure 4.6: Deflection of the meniscus vs applied voltage for various distance between plates. Dashed lines: numerical model, symbols: experimental data.

corresponds to a situation where a liquid interface is exposed to an external field $E_{0}=V / h$ at large distance. With the Maxwell stress scaling as $(V / h)^{2}$ and the Laplace pressure scaling as $\gamma / a$, we find that the stability limit should be determined by a critical electrocapillary number scaling as $a V^{2} / h^{2} \propto H \Lambda_{c}^{2}$. Here $\Lambda=(a / h) \sqrt{\varepsilon \varepsilon_{0} V^{2} / 2 \gamma h}$ describes the relative strength of electrostatic forces with respect to the Laplace pressure and $\gamma$ is the surface tension of the liquid. Hence, we expect $H \Lambda_{c}^{2}$ to approach a constant value of order unity as the system approaches the Taylor cone regime. Moreover, as it can be seen in Fig. 4.6 the critical deflection of the meniscus also saturates for large numbers of $H$. The maximal deflection of the meniscus before the instability does not exceed the value $\zeta_{c}=240 \mu m$.

In Fig. 4.7 we plot $H \Lambda_{c}^{2}$ and the critical deflection as a function of aspect ratio $H$. Here for convenience we normalize the deflection by the radius of the hole, plotting $H \xi_{c}=\zeta_{c} / a$. Dashed lines in the figure represent the numerical results. The experimental results for normalized critical deflection are in good agreement 


\section{ELECTRIC FIELD DRIVEN INSTABILITIES ON SUPERHYDROPHOBIC SURFACES}

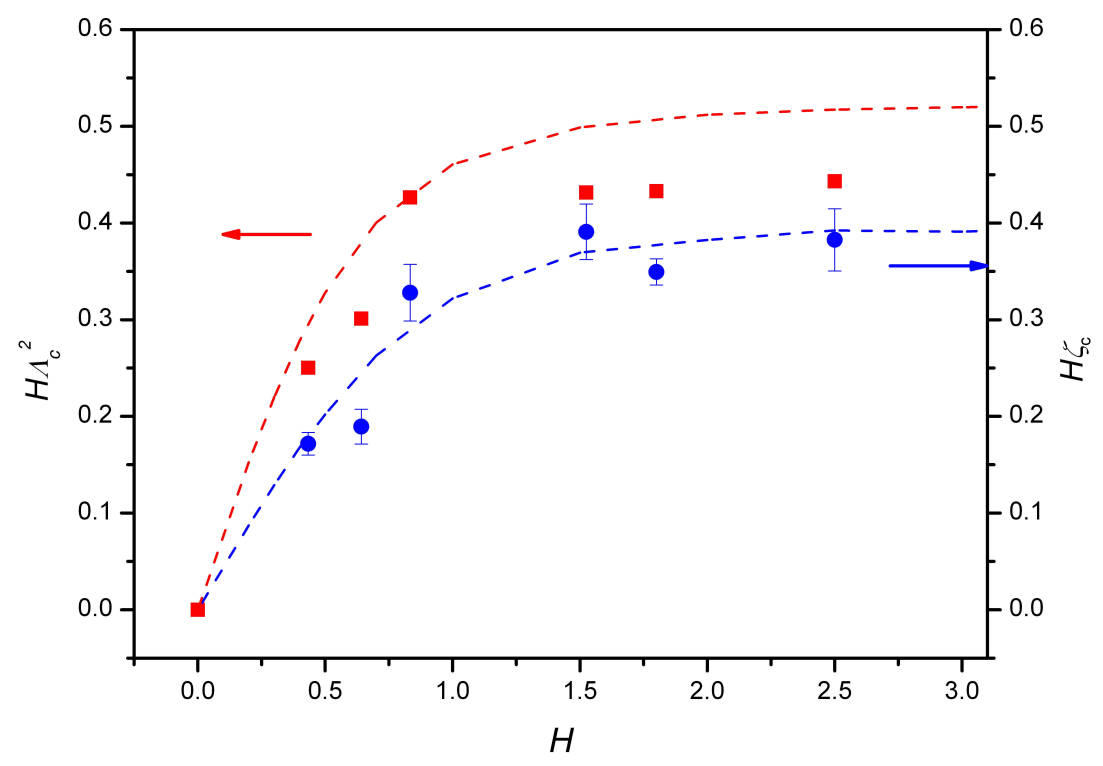

Figure 4.7: Dependence of $H \Lambda_{c}^{2}$ and $H \xi_{c}$ on aspect ratio full symbols: experimental data, empty symbols: model result.

with the numerical prediction whereas for the critical electrocapillary number we obtain smaller values than the model predictions. We attribute these deviations to the fact, that for voltages close to the critical value small variations in voltage result in large variations of the deflection (see Fig. 4.6), thus experimentally obtained values for critical voltage are smaller than the real $V_{c}$.

\subsection{Conclusion}

Our numerical and experimental study demonstrates that electrohydrodynamic instabilities are an important limiting factor for the stability of the Cassie state on superhydrophobic surfaces exposed to electric fields. Electrohydrodynamic instabilities compete with the classical transition criterion due to contact line depinning from the edges of the surface topography, which dominates for small contact angles. For depth-to-width aspect ratios of the surface topography exceeding 
$H=2$, the critical Taylor cone-like shape of the surface becomes independent of the aspect ratio. This implies a constant critical contact angle delineating depinning and electrohydrodynamic instabilities. For superhydrophobic surfaces involving geometric overhangs that suppress contact line depinning, electrohydrodynamic instabilities are likely to be the dominant instability mechanism.

We also demonstrate the nonspherical nature of the liquid-gas interface under an applied electric field. The reason for that is a nonhomogenous distribution of the electric forces along the liquid interface. This leads to non-spherical deformation of the interface (see Fig. 4.3), contrary to a pressure driven collapse on conventional superhydrophobic surfaces [63, 73], in which the shape of liquid interface is governed by only Laplace pressure. 
4. ELECTRIC FIELD DRIVEN INSTABILITIES ON SUPERHYDROPHOBIC SURFACES 


\section{Chapter 5}

\section{(Electro)-wetting of a drop on a sphere}

In this chapter, the equilibrium morphology of a drop on a sphere is analyzed as a function of contact angle and drop volume experimentally and with analytic effective interfacial energy calculations. Experimentally, a drop on a sphere geometry is realized in an oil bath by placing a water drop on a sphere coated with a dielectric, The radii of curvature of the sphere are comparable with that of the drop. Electrowetting $(E W)$ is used to change the contact angle of the water drop on the sphere. To validate the applicability of EW and the Lippman-Young equation on non-flat surfaces, we systematically investigated the response of the contact angle to the applied voltage ( $E W$ response) for various drop volumes and compared the results with the case of a planar surface. The effective interfacial energy of two competing morphologies, namely the spherically symmetric "completely engulfing" and the "partially engulfing" morphology are calculated analytically. The analytic calculations are then compared to the experimental results to confirm which morphology is energetically more favored for a given contact angle and drop volume. Our findings indicate that the "partially engulfing" morphology is always energetically more favorable. 1

\footnotetext{
${ }^{1}$ Part of this chapter has been published in "Wetting of a Drop on a Sphere", Eral, H. B. Manukyan, G. and Oh, J. M [76]. Gor Manukyan and Burak Eral have equal contribution on the paper.
} 


\section{5. (ELECTRO)-WETTING OF A DROP ON A SPHERE}

\subsection{Introduction}

Drops on curved surfaces are omnipresent in nature: dew drops on a spider web or a rain drop on a fruit provides the most intriguing display of drop morphologies on curved surfaces (Fig 5.1). The morphologies that a drop assumes on a curved surface are dictated by wettability of the substrate, which is a key parameter for addressing fundamental problems of fluid stability on complex geometries [77, 78]. The wettability of a substrate depends on the interplay between the contact angle and the geometry of the surface. For instance, a drop that partially wets a planar surface can engulf a fiber with same wettability.

The drop on a sphere geometry is not only a classic wetting geometry but also it is often encountered in industrial and fabrication processes. Such processes can benefit from the fundamental understanding of the wettability for the drop on a sphere geometry. Recently, this classic wetting geometry was evoked to synthesize complex colloidal particles by growing a colloidal particle inside a polymer drop [79]. The colloidal particle that is initially in "completely engulfing" morphology later assumes a "partially engulfing morphology" dictated by the wettability. Wettability of the polymer drop on the particle is controlled by the reaction parameters such as monomer concentration and temperature [79, 80]. Various applications in colloidal science [81, microfluidics [82, 83, 84, 85, 86], detergency [87], optofluidic lenses [88], electronic displays utilize spherical and other non-flat geometries [89, 90].

A drop on a sphere can have two topologically distinct morphologies: the "partially engulfing" morphology and the spherically symmetric "completely engulfing" morphology. These two morphologies are fundamentally different in terms of their symmetry and response to external driving such as shear or mechanical agitation. The wettability of a droplet on a spherical surface is addressed in various theoretical studies, [91, 92, 93, 94] yet an experimental setup that allows precise control over parameters that determine the wettability hence the equilibrium morphologies was missing. Previous experimental studies on spherical surfaces [95, 96] utilized different materials to vary the contact angle. These methods do not offer the required resolution to explore the range of contact angles defining the morphologies. 
For experimental realization of the drop on a sphere geometry, a water drop in an oil bath is placed on a metal sphere coated with a dielectric layer. The wettability of the drop on the sphere is tuned by electrowetting (EW). Electrowetting is an efficient way of controlling the wettability of liquids on surfaces. The effective interfacial tension, or equivalently the apparent contact angle $\theta$ of a conducting drop on a dielectric substrate is controlled by applying a potential difference $(U)$ between the conducting liquid and an electrode integrated under the dielectric layer [40]. The difference between the cosine of the apparent contact angle $\theta$ and the cosine of the microscopic contact angle $\theta_{Y}$ at the three-phase contact line (TCL) is proportional to the square of the applied potential $\left(U^{2}\right)$ by the Lippmann-Young equation (Eq. 5.1).

$$
\cos \theta=\cos \theta_{Y}+\eta, \quad \eta \equiv \frac{\varepsilon_{0} \varepsilon_{r} U^{2}}{2 \delta \gamma},
$$

where $\theta_{Y}$ is the Young's angle and the electrowetting number $\eta$ is defined by the vacuum permittivity $\varepsilon_{0}$, the dielectric constant $\varepsilon_{r}$, the dielectric layer thickness $\delta$, and the interfacial tension $\gamma$ between liquid and solid phases.

First, we confirm the applicability of the Lippmann-Young equation and EW to study the equilibrium morphology of drops on curved surfaces. For this purpose, the response of the contact angle to the applied voltage (EW response) for a drop on a spherical, a cylindrical and a planar substrate is compared. Furthermore, we investigate the EW response on curved surfaces systematically as a function of the drop volume. The volume of the drop is varied to change the relative surface curvature $\left(\kappa_{1} / \kappa_{2}\right)$, where $\kappa_{1}$ and $\kappa_{2}$ are the curvatures of drop and surface, respectively.

The validation of the Lippmann-Young equation for non-flat surfaces is required as the intrinsic curvature of the geometry and dielectric layer thickness influence the electric force distribution in the vicinity of the contact line. In the derivation of Eq. 5.1, the force distribution is obtained assuming that the supporting substrate is flat. For a non-flat surface, the electrical force distribution is altered due to the surface curvature and, as a consequence, the wettability change may deviate from the Lippmann-Young relation. Furthermore, this variation might depend on the relative surface curvature and dielectric layer thickness. 


\section{5. (ELECTRO)-WETTING OF A DROP ON A SPHERE}

It has been shown that the electrostatic force in the presence of electrowetting is concentrated near the contact line in a region in the order of the dielectric thickness [62, 97]. From this point, one can hypothesize that the curvature of the sphere will not be dramatically influencing the EW response due to large difference in dimensions considered. We provide experimental confirmation of this hypothesis stating the curvature effects can be ignored in this study.

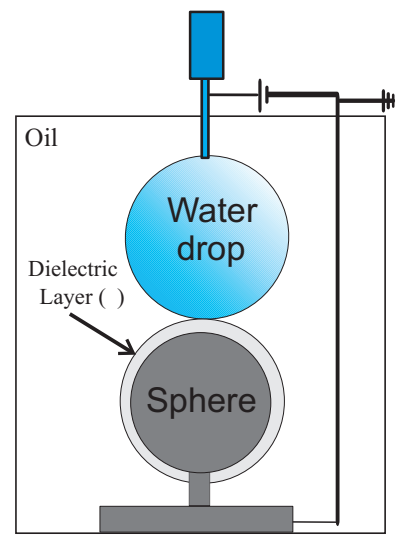

(a)

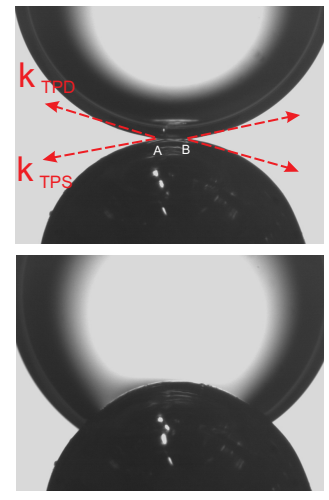

(b)

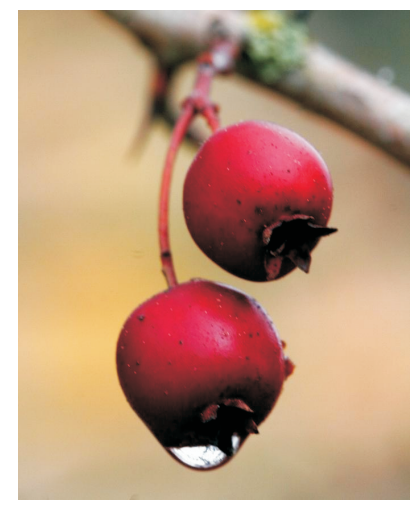

(c)

Figure 5.1: Panel (a) shows the experimental setup . The setup consists of a non-flat steel substrate coated with a dielectric layer of thickness $(\delta)$ and a water drop placed on the top, immersed in oil. The metal sphere is grounded and the water drop is connected to a power supply in our setup. Panel (b) shows images of the experimental system where the voltage is changed from 0 to $300 \mathrm{~V}$. Panel (c) presents "partially engulfing" morphology from nature, a water drop sitting on a berry courtesy of S.Dreilinger. Note that in nature gravity is ever present as opposed to experiments presented here and it induces additional asymmetry.(the gravity in the experiments is negligible due to very little difference in densities between water and silicone oil)

Secondly, we determine the equilibrium shapes of a drop on a sphere geometry experimentally and analytically relying on the aforementioned confirmation. The parameter space consisting of contact angle and drop volume is scanned to find the stable morphologies with the experimental setup shown in Fig. 5.1 . The effective interfacial energy of the equilibrium morphologies are extracted ex- 
perimentally by image processing and compared to analytical effective interfacial energy calculations. We believe that our systematic investigation will address fundamental wetting questions [17, 98, 99] where manipulation of contact angle plays an important role for complex geometries [100, 101] .

\subsection{Materials and methods}

\subsubsection{Electrowetting setup}

Our buoyancy-neutral experimental setup consists of a stainless steel sphere $(r$ $=1.5 \mathrm{~mm}$ ) coated with a dielectric layer of thickness $\delta$ (spatial variation of $\delta$ is between 2 to $4 \mu \mathrm{m}$ with mean of $3.1 \mu \mathrm{m}$ ) immersed in silicon oil (Fluka AS4 with density $\rho=1.01 \mathrm{~g} / \mathrm{cm}^{3}$ and viscosity $\left.\mu=6 \mathrm{mPa} \cdot \mathrm{s}\right)$. The experiment is performed in oil to avoid evaporation and to reduce the contact angle hysteresis as well as to prevent the buoyancy effect. The relative importance of gravity over interfacial tension is measured by the Bond number $B o=\Delta \rho g L^{2} / \gamma$ where $\Delta \rho$ is the density difference between oil and water phases, $L$ is the characteristic length given by the radius of the drop with maximum drop volume $(8 \mu \mathrm{l})$. In our density matched experimental setup $B o \approx 10^{-2}$, so the gravity effects can be ignored. A function generator (HP33250A) and an amplifier (Trek PZD350) are used to supply the required voltage. A video goniometer (OCA Dataphysics) is used to capture images. We place a water drop on top of the spherical substrates. The volume of the drop is controlled by an automated syringe pump whose needle also acts as an electrode (Fig 5.1). In these experiments, the volume of the drop $\left(V_{d r o p}\right)$ are varied from 8 to $1 \mu \mathrm{l}$.

\subsubsection{Substrate preparation}

Non-flat substrates are prepared with a cleaning procedure followed by two consecutive dip coating steps. In the first step, the steel spheres are cleaned with ethanol in an ultrasonic bath for 15 minutes and left to dry in a fume hood under ambient conditions. Secondly, the substrates are dip coated with SU-8, then the SU-8 coated substrates are placed on a hot plate for 3 minutes and finally 


\section{5. (ELECTRO)-WETTING OF A DROP ON A SPHERE}

the substrates are UV cured for 30 seconds. In the last step, the SU8 coated spheres are dip coated again with 0.6 wt.\% Teflon AF solution. The thickness of the dielectric layer is measured from scratched samples by the Scanning Electron Microscopy (SEM) after the experiments. The SEM images are given in the Supplementary Information.

\subsubsection{Image processing}

\subsubsection{Contact angle measurements}

The images of the drop on spherical substrates are captured with a CCD camera for different voltages. To measure the (apparent) contact angle $\theta$ on non-flat geometries (Fig, $5.1 \mathrm{~b}$ ), a simple thresholding algorithm is used to locate the oilwater-sphere contact line. To measure the contact angle $\theta$, two tangent vectors originating from the three-phase contact line (TCL) are needed, one tangential to the drop-oil interface: $\tilde{k}_{T P D}$ and the other tangential to the spherical substrate-oil interface: $\tilde{k}_{T P S}$. Two vectors $\left(\tilde{k}_{T P D}, \tilde{k}_{T P S}\right)$ are defined by the least square fitting method in the vicinity of the TCL ( Fig $5.1 \mathrm{~b}$ ). The angle $\pi-\theta$ between these two vectors gives us $\theta$. The procedure was repeated for the both sides of the TCL ( $\mathrm{A}$ and $\mathrm{B}$ in Fig.5.1b) and the averaged contact angle is given in this study. The error bound of contact angle measurements is calculated as $3^{\circ}$ by varying parameters of the detection algorithm systematically.

\subsubsection{Effective interfacial energy calculations from experimental data}

The effective interfacial energies of the two morphologies are calculated by extracting the surface areas of each interface from the digital images indicated in Fig 5.2. For the "partially engulfing" morphology of three phases in contact: the oil-water drop interface area (S1), the drop-substrate contact area (S2), and the oil-substrate contact area (S3) are considered. For the "completely engulfing" morphology, the sphere is not in contact with the surrounding oil medium so only oil-water drop interface and water-solid contact areas are considered.

Calculation of the effective interfacial energy for the "completely engulfing" morphology $\left(E_{1}\right)$ requires the determination of water drop-solid sphere and water drop-oil contact areas. As these contact areas are not contact angle dependent, 
provided that the drop volume and the radius of the sphere are known, $E_{1}$ can be calculated by multiplying the surface tension of the interfaces with respective contact areas.

For the "partially engulfing" morphology, contact areas $S_{1}, S_{2}$ and $S_{3}$ vary with the contact angle $\theta$. $E_{2}$ varies with respect to $\theta$ The parameters that vary with respect to $\theta$ are $\theta_{1}, \theta_{2}$ and $R$ as shown in Eq.5.2. Provided that with the radius of the solid sphere $(a)$ and the drop volume are known, the parameters $\theta_{1}, \theta_{2}$ and $R$ are calculated by simple geometric relationship once the three-phase contact line (TCL) and the centers of liquid drop $C M_{\text {drop }}$ and sphere $C M_{\text {sphere }}$ are known. More specifically, the images allow us to find the $C M_{d r o p}$ and $C M_{\text {sphere }}$ precisely by fitting a circle to the contour of the the drop and the sphere. The $\theta$ dependent $\theta_{1}, \theta_{2}$ can be calculated from the inner product of two vectors. The inner product of vector connecting $C M_{\text {drop }}$ to $C M_{\text {sphere }}$ and vector connecting $C M_{d r o p}$ to TCL gives $\theta_{2}$. The inner product of the vector connecting $C M_{d r o p}$ to $C M_{\text {sphere }}$ with the vector connecting $C M_{\text {sphere }}$ to TCL gives $\theta_{1}$. These parameters allow for calculation of contact areas S1, S2, S3 from Eq $5.3-5.5$. Once the contact areas and the respective interfacial tension values are known, the effective interfacial energy for the "partially engulfing" geometry $\left(E_{2}\right)$ is calculated.

The effective interfacial energies of two morphologies $\left(E_{1}, E_{2}\right)$ as a function of $\theta$ are later used in Eq.5.9 to calculate the effective interfacial energy difference between the two morphologies. Assuming that the spherical symmetry approximation holds, i.e. the drop shape is not disturbed by the wire and gravity.

\subsubsection{Analytical effective interfacial energy calculations}

The effective interfacial energies of the two morphologies seen in Fig 5.2 are analytically calculated as a function of the drop volume and the contact angle. For this purpose, the radius of drop $R$ in the "partially engulfing" morphology can be expressed in terms of $a, \theta_{1}, \theta_{2}$ with the spherical shape assumption.

$$
R=\frac{\sin \theta_{1}}{\sin \theta_{2}} a
$$




\section{5. (ELECTRO)-WETTING OF A DROP ON A SPHERE}

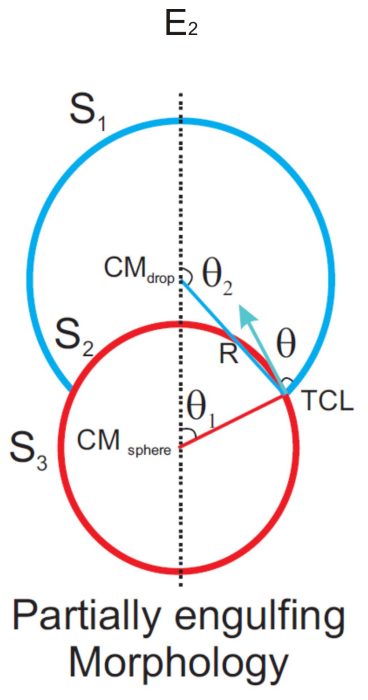

(a)

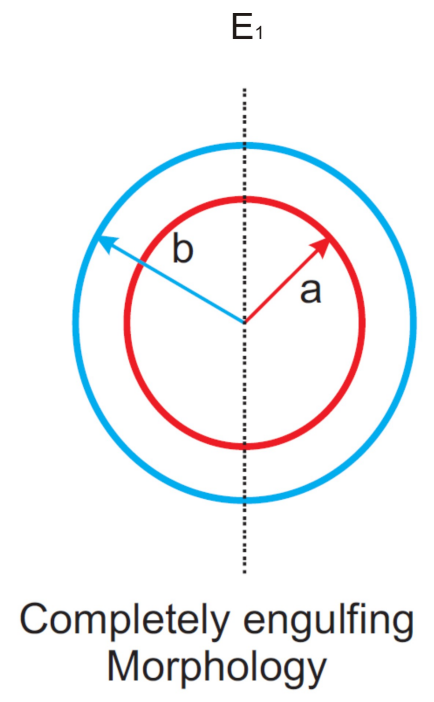

(b)

Figure 5.2: Panel (a) shows the "partially engulfing" morphology and (b) the "completely engulfing" geometry. The parameters indicated in the effective interfacial energy calculations are indicated in both panels.

here $\theta_{2}=\theta+\theta_{1}$, where $\theta$ is the contact angle and $a$ is the radius of spherical substrate. The surface area of the drop $\left(S_{1}\right)$, the drop-substrate contact area $\left(S_{2}\right)$, and the air-substrate contact area $\left(S_{3}\right)$ can be expressed as follows:

$$
\begin{aligned}
& S_{1}=2 \pi R^{2}\left(1-\cos \theta_{2}\right), \\
& S_{2}=2 \pi a^{2}\left(1-\cos \theta_{1}\right), \\
& S_{3}=2 \pi a^{2}\left(1+\cos \theta_{1}\right) .
\end{aligned}
$$

The surface area of the drop $\left(S_{4}\right)$ in contact with oil is given in "completely engulfing" morphology as

$$
S_{4}=4 \pi b^{2},
$$

where $b$ is the radius of the drop in the "completely engulfing" morphology

$$
b=\left(a^{3}+\frac{V}{4 \pi / 3}\right)^{1 / 3} .
$$


The difference $\Delta E=E_{1}-E_{2}$ between the effective interfacial energy of the completely engulfed morphology $\left(E_{1}\right)$ and the partially engulfed morphology $\left(E_{2}\right)$ is given in Eq 5.8 .

$$
\Delta E=E_{1}-E_{2}=\left(S_{4} \gamma+\left(4 \pi a^{2}\right) \gamma_{w s}\right)-\left(S_{1} \gamma+S_{2} \gamma_{w s}+S_{3} \gamma_{s o}\right)
$$

where $\gamma, \gamma_{w s}$ and $\gamma_{s o}$ denote the interfacial tension between water-oil, water-solid and solid-oil phases, respectively. The total effective interfacial energy difference can be rearranged as follows:

$$
\frac{\Delta E}{\gamma}=\left(S_{1}-S_{4}\right)+S_{3} \cos \theta
$$

When $\Delta E>0$, the partially engulfing morphology is energetically favorable compared to the completely engulfing morphology.

\subsection{Results and disscusion}

To confirm the applicability of the Lippmann-Young equation for non-flat surfaces, we focus on three parameters: (a) substrate geometry (b) drop volume and (c) voltage. The responses of the contact angle with respect to the applied voltage for different substrate geometries and drop volumes are shown in Fig 5.3 .

The influence of substrate geometry on EW response is examined for a drop of $8 \mu \mathrm{l}$ volume placed on spherical, cylindrical and planar surfaces. Fig 5.3 demonstrates how the contact angle changes as a function of the applied voltage and compares the EW response for aforementioned substrate geometries. The cosine of the contact angle $(\cos \theta)$ is plotted as a function of the non-dimensionalized EW number to account for variations in $\delta$ in Fig 5.3. The $\delta$ is extracted from SEM images. The curves follow the Lippmann-Young equation in the LippmannYoung regime followed by the saturation regime where the contact angle does not vary with applied voltage [40].

All the curves in Fig 5.3 collapse onto the Lippman-Young equation prior to saturation regime, implying that the influence of geometry on EW response is negligible as previously hypothesized. The contact angle $(\cos \theta)$ is plotted against square of the applied voltage for different drop volumes in the inset of 


\section{5. (ELECTRO)-WETTING OF A DROP ON A SPHERE}

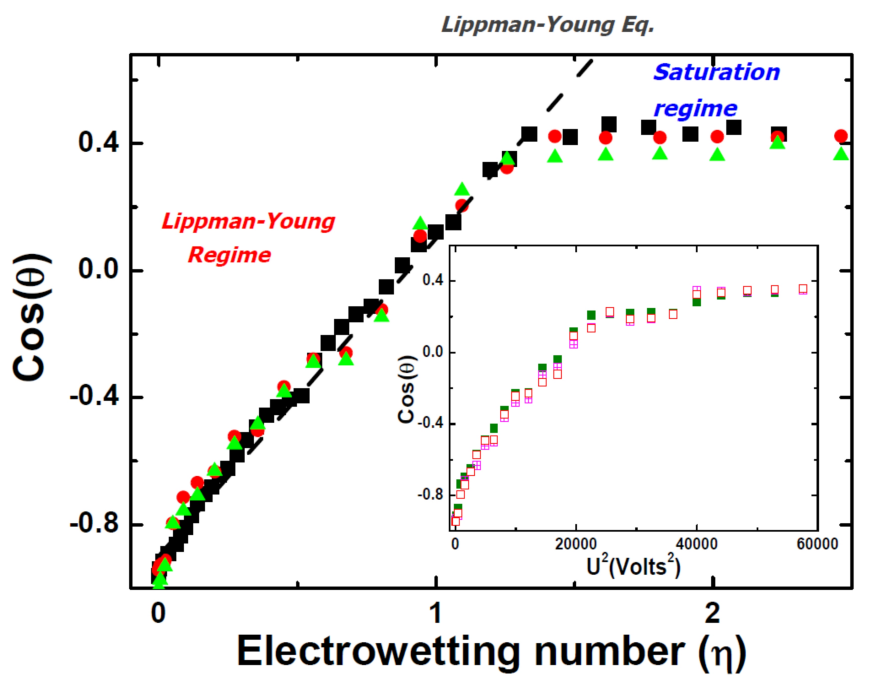

Figure 5.3: Influence of the substrate geometry and the drop volume on the response of contact angle to an applied voltage (EW response). In the main figure, the cosine of the contact angle $(\cos \theta)$ is plotted as a function of EW number $(\eta)$ for different geometries with a given drop volume $(\mathrm{V}=8 \mu \mathrm{l})$. The symbols indicate substrate geometry: cylindrical (triangle), spherical (sphere) and flat (square). The dashed line denotes the Lippmann-Young Equation. In the inset, $\cos \theta$ is plotted as a function of square of $U^{2}$ for different drop volumes placed on a spherical geometry. The symbol fill patterns denote different drop volumes $V_{\text {drop }}$ : (whole $8 \mu$ l, empty $4 \mu \mathrm{l}$ and crossed $1 \mu \mathrm{l})$, respectively.

Fig 5.3. The collapse of data for different drop volumes (denoted by symbol fill patterns in Fig, 5.3 in the Lippmann-Young regime points out that the drop volume hence relative curvature has no influence on the response of contact angle to the applied voltage for spherical substrates (in the studied range). We have also looked at the influence of drop conductivity on EW response for spherical substrates. Dependence of EW response on drop conductivity is found to follow similar behavior as for planar surface [42, 102, 103]. In Fig. 5.3 and its inset, a slight scattering and deviation from the Lippmann-Young equation is observed. This is attributed to a spatial variation in the dielectric layer thickness of samples used in experiments which we have identified to vary between 2-5 microns.

After validating the applicability of the Lippmann-Young equation and the 
reliability of EW on spherical substrates, we investigate the equilibrium morphologies by comparing the effective interfacial energies of the partially engulfing and the completely engulfing morphologies in Fig.5.2. To assess which morphology is more favorable, we calculate the effective interfacial energy difference between these two morphologies (Eq.5.8). When $\Delta E \geq 0$, the completely engulfing morphology is the energetically favorable and $\Delta E<0$ the partially engulfing is the favorable.

Evaluation of the drop morphology as a function of contact angle is monitored by plotting the projected distance $d(\theta)$ between the center of mass $C M_{\text {sphere }}$ of the solid sphere and the center of mass of the drop $C M_{\text {droplet }}$ at a given contact angle in Fig. 5.4 from experimental images (Fig 5.4 c). With decreasing contact angle, $d(\theta)$ gradually decreases and the drop partially engulfs more of the sphere for all drop volumes. To characterize equilibrium shapes for different drop volumes, we defined a symmetry parameter, $\lambda$, which denotes the relative distance $d(\theta)$ at a given contact angle normalized by the same distance at the Young's angle $d\left(\theta_{Y}\right)$ in Eq.5.10. For a given contact angle, $\lambda$ is determined from experimental images as follows: $C M_{\text {drop }}$ and $C M_{\text {sphere }}$ are determined by identifying the contour of the drop-sphere via track holding and fitting a circle above and below TCL. The distance between $C M_{d r o p}$ and $C M_{\text {sphere }}$ determines $d(\theta)$ for a given contact angle. The ratio of this distance at a given contact angle to this distance at Young angle: $d\left(\theta_{Y}\right)$ gives the symmetry parameter $(\mathrm{Eq} 5.10)$. The inset schematic in Fig $5.4 \mathrm{~b}$ demonstrates the symmetry parameter.

$$
\lambda=\frac{d(\theta)}{d\left(\theta_{Y}\right)}
$$

For $\lambda \cong 0$ the drop is in "completely engulfing" morphology for $\lambda=1$ the drop is in its equilibrium morphology without any applied voltage. Fig. 5.4b demonstrates the variation of $\lambda$ as a function of contact angle $(\theta)$ for aforementioned drop volumes. The curves of different drop volumes collapse onto a single curve and none of the curves reaches $\lambda \simeq 0$ hence the "partially engulfing" morphology is always the preferred morphology for this $\theta$ range.

The effective interfacial energy difference $(\Delta E)$ is calculated analytically and from experimental data as described in detail in the Methods section. Fig 5.5 


\section{5. (ELECTRO)-WETTING OF A DROP ON A SPHERE}

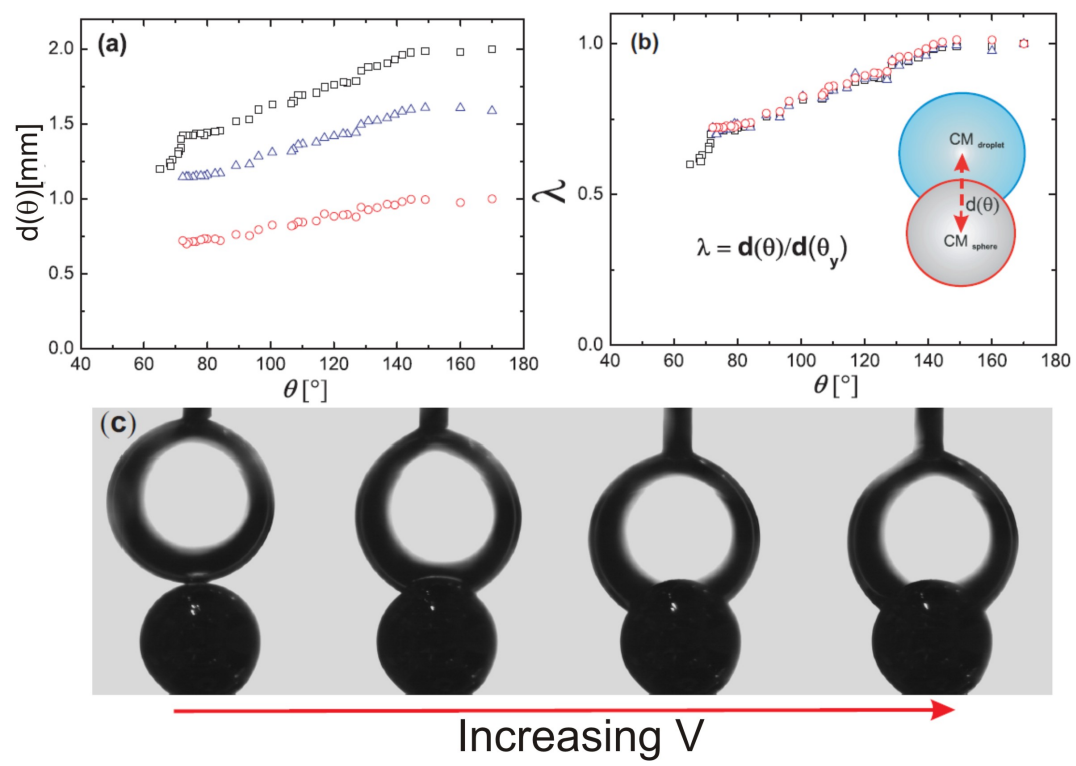

Figure 5.4: Evolution of the drop morphology as a function of drop volume $V_{d r o p}$ and contact angle $\theta$. Panel (a) provides the distance between the center of mass of drop and the sphere $(d(\theta))$ as a function of $\theta$ for different drop volumes. Panel (b) gives the symmetry parameter $(\lambda)$ vs. $\theta$. Different symbols correspond to drop volumes $(1 \mu l(\bigcirc), 4 \mu l(\triangle), 8 \mu l(\square)$ respectively). The symmetry parameter $(\lambda)$ is defined as the distance $d(\theta)$ between the center of mass of the sphere and the drop at a given $\theta$ normalized $\operatorname{by} d\left(\theta_{Y}\right)$ : the distance when $\theta$ is equal to $\theta_{Y}$. Inset in panel (b) demonstrates the $d(\theta)$ and symmetry parameter. Panel (c) provides an overview of the experimental images for various $\theta$.

shows the effective interfacial energy difference normalized by $4 \pi a^{2} \gamma$ as a function of the contact angle for different drop volumes. The solid lines denote the analytical effective interfacial energy calculations whereas the discrete points indicate the effective interfacial energies calculated from the experimental data.

For all the effective interfacial energy calculations considered here, the effective interfacial energy difference indicates that the favorable morphology is the "partially engulfing" morphology irrespective of the drop volume and the contact angle. These results are in agreement with the effective interfacial energy analysis given in Fig 5.4 . Only when the contact angles approach to zero, the energy difference also converges to zero. This means that the surface energy of 
the wetted area is the same with that of non-wetted area $\left(\gamma+\gamma_{s w}=\gamma_{s o}\right)$. Due to contact angle saturation phenomena, very low contact angles cannot be reached experimentally. Yet, the analytical effective interfacial energy calculations show that the two morphologies have equal effective interfacial energies indicating that the two morphologies are equally favorable for zero contact angle. It implies that with large enough drops and zero contact angle a transition from one morphology to the other is theoretically possible.

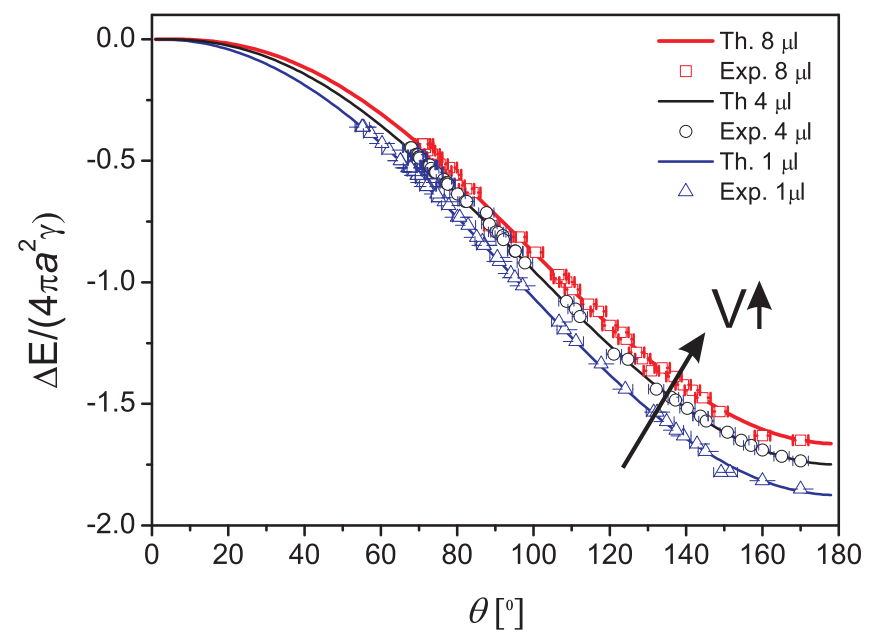

Figure 5.5: Normalized effective interfacial energy is plotted as a function of contact angle for different drop volumes used in experiment. Continuous lines indicate the theoretical and the discrete points of same color indicate experimental effective interfacial energies extracted from image processing. Different symbols correspond to drop volumes $(1 \mu l(\triangle), 4 \mu l(\bigcirc), 8 \mu l(\square)$ respectively.

In this study, the upper and lower bounds of drop volume considered are defined by the limitations of the experimental setup and the associated physics. The smallest volume utilized is bound by the size of wire in conventional EW setup (The drop diameter has to be a lot bigger than the wire). The upper limit is bound by the capillary limit. Provided that we are not limited by the experimental parameters these two extreme limits may provide interesting insights. For the generality of our results, it is relevant to discuss these extreme cases: ( (i) $V_{\text {drop }}>>V_{\text {sphere }}$ and (ii) $V_{\text {drop }}<<V_{\text {sphere }}$ ). In case (i), from the effective interfacial energy calculations, we can deduce that the larger the ratio of $V_{d r o p}$ to 


\section{5. (ELECTRO)-WETTING OF A DROP ON A SPHERE}

$V_{\text {sphere }}$ the smaller the difference $\Delta E$. This points out that the effective interfacial energy of two states become comparable at all contact angles. Less energy is required to jump from one morphology to the other upon application of flow or mechanic agitation. For case (ii), the transition becomes rather difficult and the systems resembles a flat geometry.

The major source of error in the calculation of the effective interfacial energy from experiments is the determination of the contact angle. The measurement error is within $3^{\circ}$. As the experimental and theoretical values collapse for all the drop volumes, effects that deform the drop such as contact angle hysteresis, gravity and wire are not significant.

Utilizing EW to study fundamental wetting problems in complex geometries provides unmatched control over the contact angle. Yet the limitations in EW should not be neglected. The contact angle saturation does not allow reaching contact angles smaller that $35^{\circ}$. This limitation can be avoided by evoking inverse EW scheme where a oil drop is immersed in water yet it has to be identified as a limitation of the method [104]. Conventional and inverse EW schemes can be used in parallel to reach larger contact angle ranges. Special care has to be taken for the conventional EW setup we utilized, as the wire has to be much smaller than the drop to minimize its effect on the symmetry of the system. Interdigitated electrode or inverse EW setups can be evoked to bypass this effect. In our study, we monitored the symmetry of the drop and manually interfered by moving the wire when the symmetry is compromised.

Our results clarify the wetting behavior of a liquid drop on a spherical surface. Comparing this behavior to the wettability of a liquid drop on a infinitely long cylindrical fiber points to some interesting differences. Fibers can be fully engulfed with a nonzero contact angle where the contact angle has to be infinitesimally small for a drop on a sphere for complete engulfment [87, 104, 105]. The critical contact angle, at which a drop engulfs a fiber, depends on the drop volume whereas for the drop on a sphere geometry such critical contact angle is not drop volume dependent. 


\subsection{Conclusions}

In the present chapter, we first validated EW as a tool to study wetting problems of non-flat geometries. Later on, relying of this validity, we confirmed the equilibrium morphologies of a classic wetting geometry: drop on a sphere as a function of governing variables drop volume and contact angle, experimentally and analytically.

For validation purposes, the influence of substrate geometry on the EW response is studied systematically considering the effect of drop volume hence relative curvature. For different drop volumes corresponding to different relative drop to substrate curvatures, the EW response stayed the same, indicating that in the experimental parameter regime covered, curvature effects can be ignored. Comparing the response of contact angle to the EW number $(\eta)$ for flat, cylindrical and spherical geometries, we conclude that substrate geometry has no influence on the EW response. Our results indicate that the Lippmann-Young equation holds for spherical substrates as for flat substrates within the experimental parameters examined. Furthermore, we hypothesize that the effect of substrate curvature can be ignored for cases where the dielectric layer thickness is much smaller than the characteristic dimension of the geometry such as the radius of curvature of surface $\left(\delta \ll \kappa_{2}\right)$.

Relying on applicability of EW on non-flat surfaces, the equilibrium morphologies of two competing geometries is studied by calculating the effective interfacial energy of both morphologies analytically and experimentally. Our results show than the "partially engulfing" morphology is the energetically more favorable and the mechanically stable morphology as the absolute effective interfacial energy of the engulfing morphology is greater that the "partially engulfing" morphology under all conditions. Only for vanishing contact angles, the effective interfacial energy of the "completely engulfing" morphology is comparable to the "partially engulfing" morphology. It is noteworthy to mention that application of EW to wetting problems in complex geometries opens alleys to explore industrially relevant problems such as mechanical stability of liquid bridges between non-flat geometries such as spheres or fibers. 
5. (ELECTRO)-WETTING OF A DROP ON A SPHERE 


\section{Chapter 6}

\section{Direct observation of non equilibrium electroosmotic instability}

\subsection{Abstract}

In this chapter we present the first direct experimental visualization of a theoretically predicted hydrodynamic instability of ionic conduction from a binary electrolyte into a charge selective solid. This instability develops above a critical voltage applied to a thin rectangular transparent concentration polarization cell containing a copper sulfate solution flanked by a copper anode and a cation selective membrane. At steady state, upon the passage a DC current, current/voltage dependence exhibits a characteristic saturation at the limiting current. Upon a further increase of voltage, current increases marking the transition to the overlimiting conductance regime. We show that this transition is mediated by the appearance of a vortical flow that increases with the applied voltage in the overlimiting regime 1

${ }^{1}$ Part of this chapter has been published in "Direct Observation of a Nonequilibrium ElectroOsmotic Instability", S. M. Rubinstein, G. Manukyan, A. Staicu, I. Rubinstein, B. Zaltzman, R. G. H. Lammertink, F. Mugele, and M. Wessling [106]. 


\section{DIRECT OBSERVATION OF NON EQUILIBRIUM ELECTROOSMOTIC INSTABILITY}

\subsection{Introduction}

Microscale fluid flows commonly arise when a DC current passes through the diffusion layers of binary ionic solutions close to charge selective solids, such as electrodes [107], ion exchange granules [108, 109, 110] or membranes [111, 112], and arrays of nanochannels [113. Under conditions of extreme diffusion limitation (concentration polarization (CP) near the limiting current [45]), these flows provide an additional ionic transport mechanism. This mechanism is essential for the operation of nanofluidic preconcentrators [111, 112] and overlimiting electrodialysis [114, 115, 116]. On short length scales, in the absence of free interfaces, these flows are not driven by gravity or surface tension but rather by the electric force acting upon the space charge of the nanometers thick interfacial electric double layer (EDL). Slip-like fluid flow induced by this force is known as electroosmosis (EO).

There are two regimes of EO, corresponding to different states of the EDL and controlled by the nonequilibrium voltage drop (overvoltage) across it [46, 117]. The two are quasiequilibrium [118, 119, 120, 121, and nonequilibrium [46, 108, 109, 110, 117, 122, 123] EO. While both result from the action of a tangential electric field upon the space charge of EDL, the first relates to the charge of quasiequilibrium EDL, whereas the second to the extended space charge of nonequilibrium EDL. The nonequilibrium EDL develops in the course of CP near the limiting current.

According to a recent theory [46, 117], a novel critical instability of quiescent ionic conduction related to the extended charge EO stands behind the overlimiting conductance through a planar ion exchange membrane. In the course of 1D conduction through a planar layer an electrolyte concentration gradient forms. Its related electric force does not impair mechanical equilibrium in the system that remains stable as long as EDL retains its quasiequilibrium structure. With the increase of voltage, the system moves away from quasiequilibrium and an extended space charge develops in the EDL. EO slip related to this extended space charge renders the quiescent conduction unstable [46, 117, 124]. This instability of $1 \mathrm{D}$ ionic conduction is reminiscent of instabilities in 1D thermal conduction, such as the Rayleigh-Benard and Marangoni instabilities. While reports of the 
underlying extended space charge EO [108, 109, 110] and, possibly its related flow patterns [107] might have been numerous, to the best of our knowledge, no experimental evidence of this instability existed. Here we report on a direct observation of such instability.

\subsection{Materials and methods}

Experiments are conducted with a transparent PMMA version of the 'quiet' horizontal cell (Fig. 6.1), previously employed for CP studies [114, 115, 124] - The cell is sealed at the bottom by a massive polished copper anode, while a cation-exchange membrane (CEM: Neosepta CMX, Tokuyama Soda Ltd., thickness $d=170 \mu \mathrm{m}$ ) seals its top. A0.01 normal degassed copper sulfate solution is used as the working electrolyte. The cross section of the anode, membrane and cell is $2 \times 2 \mathrm{~mm}$, while the cell height is $0.5 \mathrm{~mm}$. Above the membrane, a large cathode compartment is sealed by a massive copper cathode. The cathode surface area is much larger than that of the anode and membrane. This guarantees that all CP is localized at the membrane [124].

To observe the fluid flow inside the cell, the electrolyte solution is seeded with $1 \mu \mathrm{m}$ neutrally-buoyant polystyrene tracer particles (Invitrogen Fluospheres, density $\rho=1.05 \mathrm{~g} / \mathrm{ml}$ ). The flow inside the cell is visualized (see the enlarged side-view of the cell in Fig. 6.1 using a long distance microscope coupled to a 12 bit Pixelfly megapixel video-camera. The depth of field of the microscope is sufficiently narrow to permit focusing at different vertical planes of the cell.

Current response $I$ to a voltage bias $V$ is measured over a small (330 $)$ resistor in series with the cell. Under all experimental conditions the voltage drop across the resistor is $<1 \%$ of the total voltage applied to the cell. Two protocols are used for varying the voltage. In the first, voltage is continuously increased up to a maximum value, $V_{\max }$, well within the overlimiting regime (Fig. 6.2. . In the second protocol, voltage is raised in small jumps. The typical holding time between consecutive jumps is 60 seconds (Fig. 6.3). We find that there is no qualitative difference between the systems response to the two protocols, the latter, however, is useful for analyzing the relaxation dynamics of the system. 


\section{DIRECT OBSERVATION OF NON EQUILIBRIUM ELECTROOSMOTIC INSTABILITY}

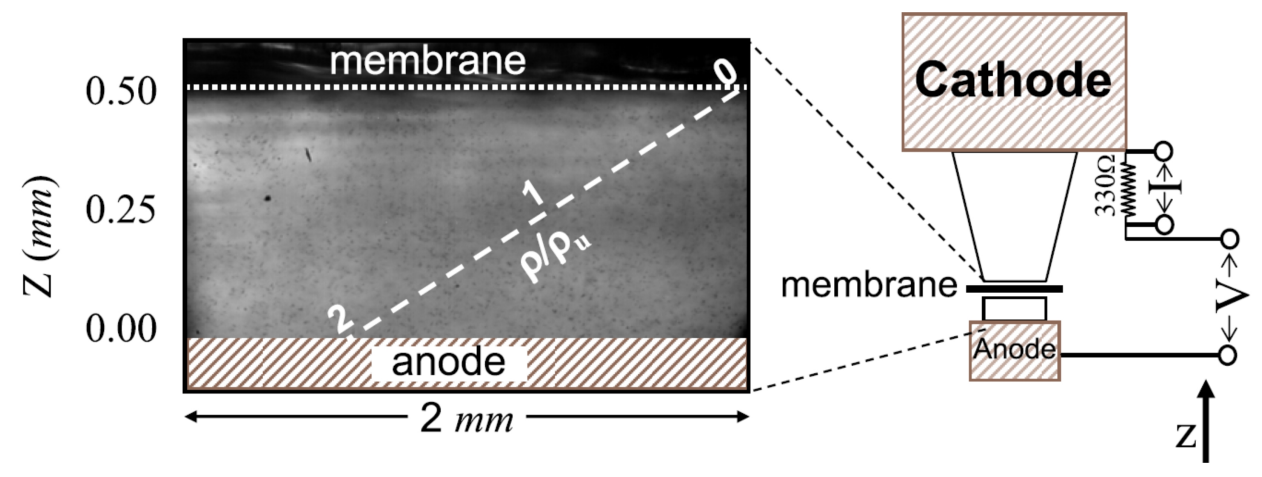

Figure 6.1: A schematic view of the experimental system. A horizontal transparent PMMA cell is sealed by a massive polished copper anode and a cation exchange membrane at the bottom and top respectively. Above the membrane, a large compartment is sealed by a massive copper cathode. The voltage bias, $V$, is applied directly to cell in series with a $330 \Omega$ resistor over which the current response, $I$, is measured. The electrolyte solution is seeded with $1 \mu \mathrm{m}$ polystyrene tracer particles. A typical enlarged view is shown on the left. The dashed line illustrates the $\mathrm{CP}$ profile at the limiting current.

\subsection{Results and discussion}

Under a DC current, copper ions dissolve from the anode, pass through the membrane and, upon their reduction at the cathode, deposit on it without any additional chemical reactions involved in the process. Due to the much higher conductivity of the anode and the membrane compared to the solution, in the absence of motion, the electric field in the anodic compartment is directed strictly upwards and is independent of the lateral coordinate. Correspondingly, also the electrolyte concentration varies in the vertical direction only, increasing at the anode and dropping at the membrane. As a result, a purely 1D conduction state develops in this compartment with the electrolyte concentration approaching a linear distribution with a slope proportional to the magnitude of the electric current (Fig. 6.1). The overall amount of copper in both compartments remains unchanged throughout the process. A typical current-voltage curve is presented in Fig. 6.2. Voltage is increased continuously up to $1.2 \mathrm{~V}$ at a rate of $3 \mathrm{mV} / \mathrm{sec}$. Three regions are distinguishable in this curve: (1) The 'ohmic' (underlimiting) 


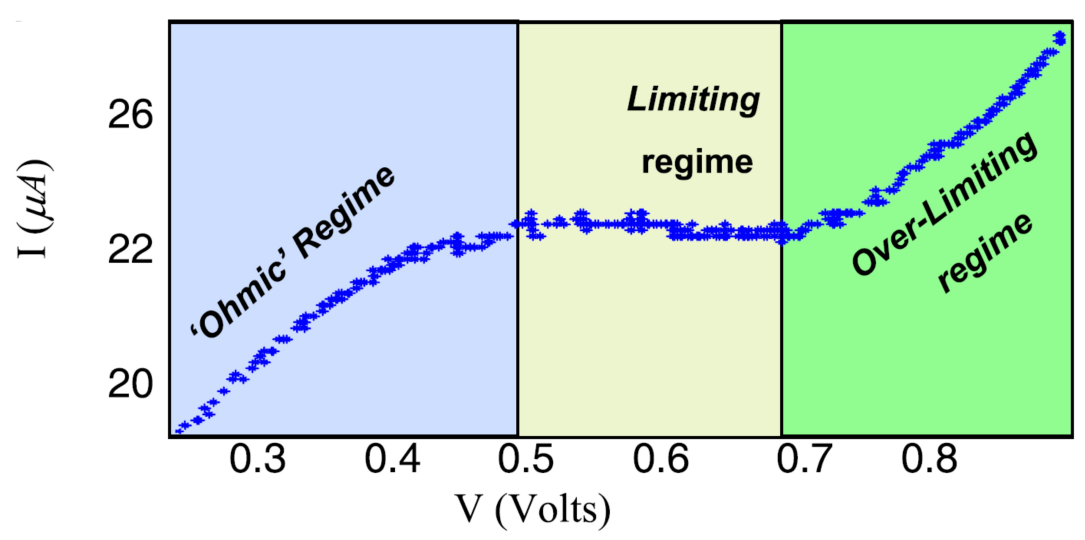

Figure 6.2: A typical $I-V$ curve measured by continuously increasing $V$ at an average rate of $3 \mathrm{mV} / \mathrm{sec}$. Three regimes are highlighted; The 'ohmic' underlimiting, limiting and overlimiting regime.

low current region followed by (2) saturation of the curve at the "limiting current" caused by the diffusion limitation of ionic transport, and (3) an inflection and transition to the overlimiting region. These three regions are typical of most current-voltage curves of CEM [114, 115], 124].

A time series of the current passing through the membrane is shown in Fig. 6.3. In this experiment voltage is increased in small jumps of $0.1 \mathrm{~V}$ once every minute (black curve). The three regions corresponding to those discussed in Fig. 6.2 are clearly visible. The notable variations in the relaxation dynamics, however, provide additional insight into the characteristic processes dominating each region. Usually, following a rapid rise in voltage, the current first 'jumps' to a higher value, and then decays towards a steady-state one. The nature by which current increases following the first two jumps is different. Rather than decaying, the current continues to increase in an almost linear rate of $\sim 55 \mathrm{nV} / \mathrm{sec}$ after the first voltage step and $\sim 30 \mathrm{nV} / \mathrm{sec}$ for the second. This increase is most likely owed to de-passivation of the anode (gradual de-blocking under current of the anode from the oxide film). The exponential time relaxation typical for the following two voltage jumps (Fig. 6.3 b), corresponding to under-limiting regime and the very beginning of the limiting current plateau, is owed to the late stage of electrodiffusional (ED) relaxation of the concentration to the linear steady 


\section{DIRECT OBSERVATION OF NON EQUILIBRIUM ELECTROOSMOTIC INSTABILITY}

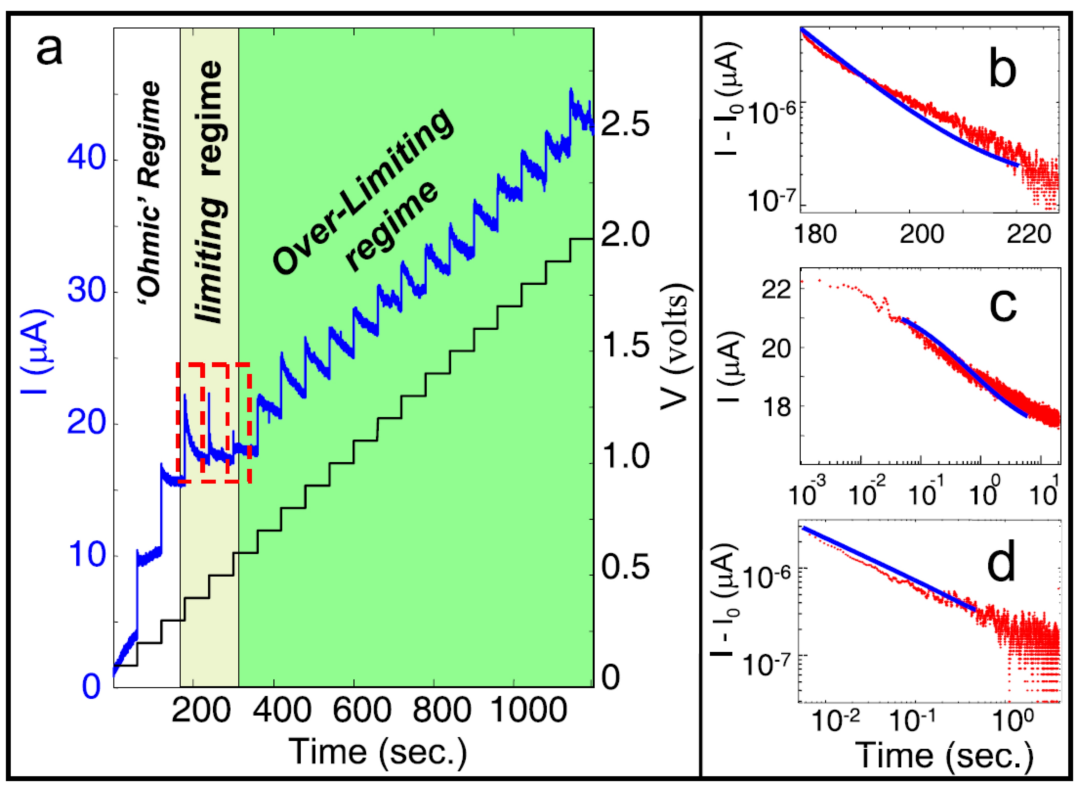

Figure 6.3: Variations in the current relaxation dynamics. (a) A time series of the current(blue) passing through the membrane. Voltage (black line) is increased in small jumps of $0.1 \mathrm{~V}$ once every minute. Three regions are highlighted as in Fig. 6.2. Above $10 \mu \mathrm{A}$ An increase in voltage is followed a rapid rise in current and then slower relaxation. Three relaxation events, within the limiting region are highlighted by dashed rectangles and correspond, from left to right to (b), (c) and (d), respectively. (b) ED relaxation of concentration to a new linear steady state profile leads to an exponential decay. (c) Toward the establishment of limiting regime relaxation is characterized by quasilogarithmic decay. (d) At the developed limiting regime the time decay changes from exponential to a power-law with a measured exponent of $-1 / 2$. Continuous lines in b-d follow from time-dependent 1D ED simulations.

state profile. Towards the end of the limiting regime, the time decay changes from exponential to a power-law with a measured exponent of $-1 / 2$ (Fig. 6.3 d). Such decay is typical to initial stages of ED concentration relaxation. Within the overlimiting regime (Fig. 6.3 a) we observe a range of linear current decay. This type of decay is not consistent with 1D ED and is suggestive of an additional transport mechanism, such as electroconvection (EC).

Summarizing, the verbal picture for the sequence of events in the underlimit- 
ing - limiting regimes, supported by our 1D quiescent ED calculations (solid lines in Figs. 6.3 b-d), is as follows: The increase of voltage instantaneously results in the increase of current due to increase of the electric field. Below, or at the very beginning of the limiting current plateau, the increase of current induces an instantaneous decrease of the interface concentration. This decrease is necessary to guarantee a zero anion flux through the interface. While the immediate response is always a $t^{-1 / 2}$ decay, it is rapidly overcome by an exponential decay. This slower decay marks the approach to a new steady state, characterized by a larger slope of the concentration profile and a larger current. At the developed limiting current, with the interface concentration at its' limiting low value the same limiting macroscopic steady state corresponds to different voltages. Applying a higher voltage, and its' corresponding instantaneous current increase, induces a local increase of the slope of concentration profile near the membrane. The $1 / \sqrt{t}$ current decay characterizes the relaxation of this disturbance, whose only result is the increase of the EDL thickness, accompanied only by a minor change in the slope of the concentration profile, undetectable on the macroscopic scale. The transition from exponential to $1 / \sqrt{t}$ is typified by the intermediate logarithmic time dependence (Fig. 6.3 c).

The transition to the overlimiting regime coincides with the voltage threshold above which vortical structures (with wavenumber $2 \mathrm{~mm}^{-1}$ compared to the range 1.5 to $2 \mathrm{~mm}^{-1}$ obtained in numerical simulation for a realistic Debye length) appear in the flow near the membrane surface. The sequence of events, as the voltage is varied is shown in Fig. 6.4 . First, at small voltages $(\leq 0.4 \mathrm{~V})$, negligible flow activity is observed. Above a critical value, the tracer particles are entrained in a streak of vertical rolls, originating at the membrane surface. We characterize the size of the vortical structures through the average distance from the membrane to the bulk of undisturbed particles, forming a clearly visible boundary in Fig.3a. The result is shown in Fig. $6.4 \mathrm{c}$ : the size of the rolls increases approximately linearly with the voltage, in a fashion similar to the growth in the electric current. Comparison to the current-voltage curve in Fig. 6.3 b reveals that the vortical structures appear at the onset of the overlimiting regime and suggests the two are manifestations of the same physical instability phenomenon. 


\section{DIRECT OBSERVATION OF NON EQUILIBRIUM ELECTROOSMOTIC INSTABILITY}
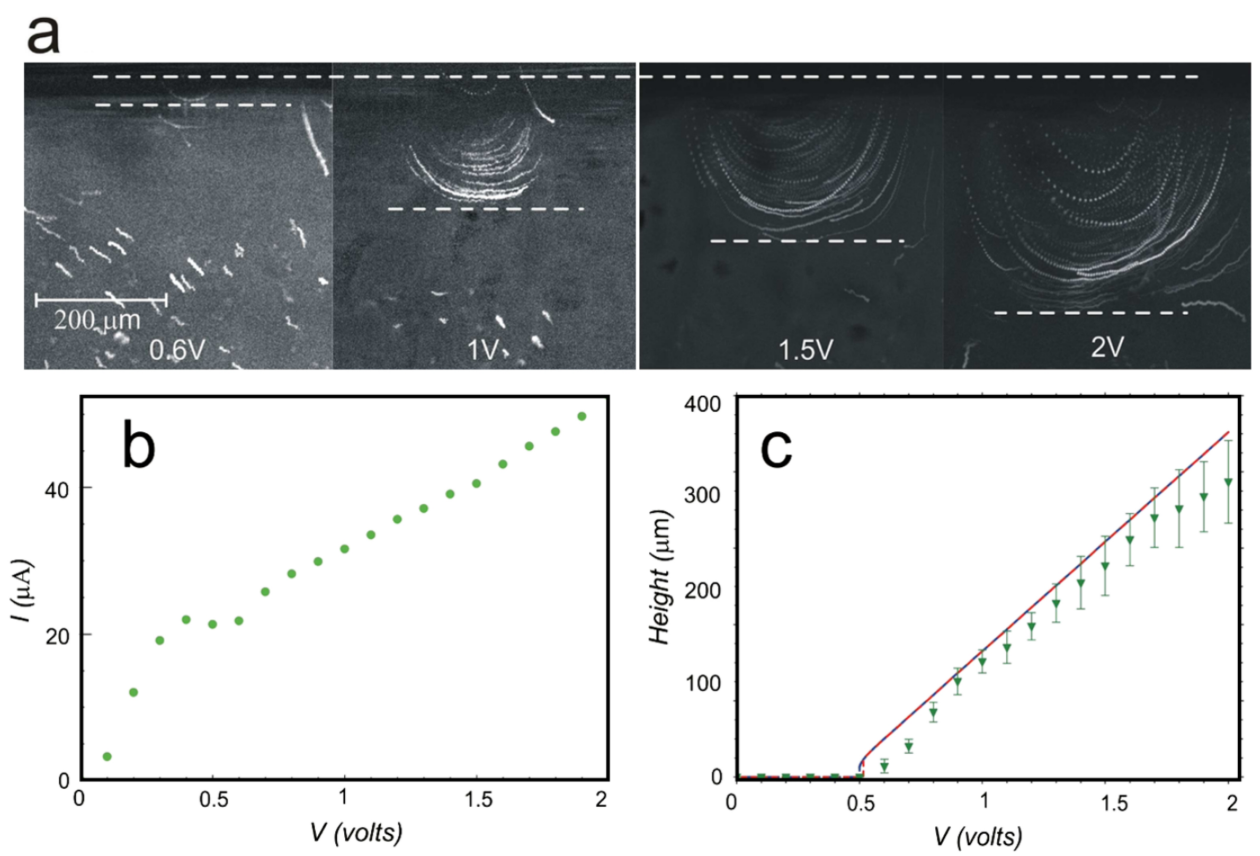

Figure 6.4: (a) Time-lapse snapshots of the experimental cell seeded with tracer particles showing 'quasi-steady-state' streamlines. The membrane is situated at the top boundary of each image; for each snapshot the applied voltage (Volts) is indicated. (b) Corresponding current-voltage curve (c) The measured (dotted line) and numerically computed (solid line) average size (height) of the vortical structures increases approximately linearly with the value of the bias voltage. Their appearance coincides with the shoulder of the curve in (b)

Numerical finite difference simulations of 2D nonlinear EC were carried out for the universal limiting formulation [46, 117] for a realistic value of dimensionless Debye length, $10^{-6}$ (Fig. 6.5). In addition to recovering the aforementioned features of 1D ED (Figs. 6.3 b-d), for developed EC in the overlimiting region, these calculations recover a long range of nearly linear time response (Fig. 6.5d) reminiscent of that in the overlimiting regime in Fig. 6.3 a. We note that EC yields formation of stirred bulk flanked by two diffusion layers with voltage dependent thickness at the solid/liquid interfaces. On the other hand, for the vicinity of bifurcation, these calculations suggest that the nonequilibrium EO instability of steady state CP is of subcritical type, with its characteristic current hysteresis. 
The calculated linear growth of average velocity with voltage, following the jump at the instability threshold, compares with that in Fig. 6.4.
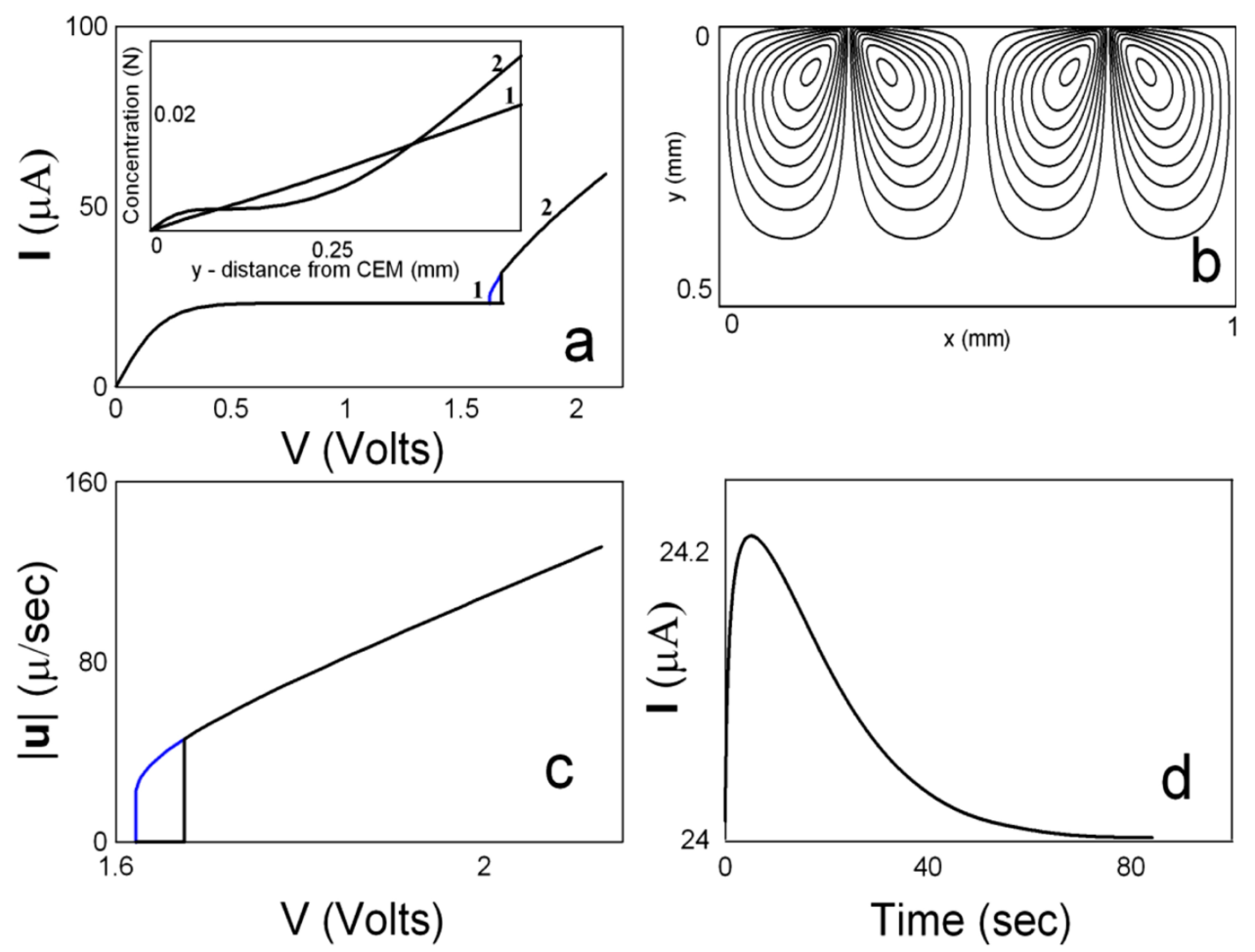

Figure 6.5: Numerical simulation of EC for dimensionless Debye length $10^{-6}$ showing hysteresis: black line - way up, blue line - way down. (a) Current/voltage dependence. (Inset: Laterally averaged concentration profiles for two voltages corresponding to the limiting (1) and overlimiting (2) currents); (b) flow streamlines' pattern; (c) voltage dependence of the absolute value of the dimensionless linear flow velocity averaged over the diffusion layer; (d) current's relaxation in the overlimiting regime

\subsection{Conclusions}

This work offers a direct experimental evidence of the theoretically predicted hydrodynamic instability of 1D ionic conduction [46, 117]. The related critical onset of the flow should be clearly distinguished from that in a multidimensional set- 


\section{DIRECT OBSERVATION OF NON EQUILIBRIUM ELECTROOSMOTIC INSTABILITY}

ting (e.g., at a curved interface corresponding to a spherical ion exchange granule [108, 109, 110] or undulated membrane surface [116], conductively heterogeneous membrane surface [125] or array of nanochannels [113]). In these cases any kind of EO initiates a flow in a thresholdless manner, that is, right upon application of an electric field. Hence, while qualitatively nonclassical EO may be recognized by its higher velocities, the precise type of the slip can only be identified by studying the dependence of velocity on the electric field. Thus, a linear dependence is expected in the classical case of quasiequilibrium EO at an originally charged interface [126] and a quadratic dependence for quasiequilibrium induced charge EO at an originally noncharged interface [119, 120, 121]. On the other hand, for the extended charge EO, cubic field dependence is expected, switching at higher voltage to a quadratic one with the tangential current density variation as a driving factor instead of the electric field [46, 117]. Namely, this type of flow has been reported recently at an array of nanochannels [113]. Likely, many more reported flows, attributed in the past to other mechanisms, belonged in fact to this category [111, 112. 


\section{Chapter 7}

\section{Summary and outlook}

\subsection{Summary}

The results described Chapter 2 demonstrate the flexibility and the control of superhydrophobicity that can be achieved using electric fields. At low voltages, the reversible bending of the micromenisci allows for detailed tuning of both hydrodynamic slip [66] and of optical diffraction from such surfaces [55].For the irreversible transition to the Wenzel state, we clearly identified that depinning of the contact lines determines the critical voltage. Combined with the criterion for the co-existence of the Cassie-Baxter state and the Wenzel state, in Chapter 3 we have demonstrated local and reversible switching between the two competing wetting morphologies. Howewer the local reversible switching presented in Chapter 3 needs more systematic study. It was also shown that the shape oscillations of the droplet under low frequency ac electric filed $(<100 \mathrm{~Hz})$ are able to switch the droplet from the partial Wenzel to the Cassie-Baxter state too.

Our numerical and experimental study in Chapter 4 shows that electrohydrodynamic instabilities are an important limiting factor for the stability of the Cassie-Baxter state on superhydrophobic surfaces exposed to electric fields. Electrohydrodynamic instabilities compete with the classical transition criterion due to contact line depinning from the edges of the surface topography, which dominates for small contact angles. For depth-to-width aspect ratios of the surface topography exceeding 2 , the critical Taylor cone-like shape of the surface becomes independent of the aspect ratio. This implies a constant critical contact angle 


\section{SUMMARY AND OUTLOOK}

delineating depinning and electrohydrodynamic instabilities. For superhydrophobic surfaces involving geometric overhangs that suppress contact line depinning, electrohydrodynamic instabilities are likely to be the dominant instability mechanism.

We also demonstrate the nonspherical nature of the liquid-gas interface under an applied electric field. The reason for that is a nonhomogenous distribution of the electric forces along the liquid interface. This leads to non-spherical deformation of the interface, contrary to a pressure driven collapse on conventional superhydrophobic surfaces [63, 73], in which the shape of liquid interface is governed by only Laplace pressure.

In the Chapter 5, we first validated electrowetting as a tool to study wetting problems of non-flat geometries. Later on, relying of this validity, we confirmed the equilibrium morphologies of a classic wetting geometry: drop on a sphere as a function of governing variables drop volume and contact angle, experimentally and analytically.

For validation purposes, the influence of substrate geometry on the electrowetting response is studied systematically considering the effect of drop volume hence relative curvature. For different drop volumes corresponding to different relative drop to substrate curvatures, the electrowetting response stayed the same, indicating that in the experimental parameter regime covered, curvature effects can be ignored. Comparing the response of contact angle to the electrowetting number $(\eta)$ for flat, cylindrical and spherical geometries, we conclude that substrate geometry has no influence on the electrowetting response. Our results indicate that the Lippmann-Young equation holds for spherical substrates as for flat substrates within the experimental parameters examined. Furthermore, we hypothesize that the effect of substrate curvature can be ignored for cases where the dielectric layer thickness is much smaller than the characteristic dimension of the geometry such as the radius of curvature of surface $\left(\delta \ll \kappa_{2}\right)$.

Relying on applicability of the electrowetting on non-flat surfaces, the equilibrium morphologies of two competing geometries is studied by calculating the effective interfacial energy of both morphologies analytically and experimentally. Our results show than the "partially engulfing" morphology is the energetically more favorable and the mechanically stable morphology as the absolute effective 
interfacial energy of the engulfing morphology is greater that the "partially engulfing" morphology under all conditions. Only for vanishing contact angles, the effective interfacial energy of the "completely engulfing" morphology is comparable to the "partially engulfing" morphology. It is noteworthy to mention that application of electrowetting to wetting problems in complex geometries opens alleys to explore industrially relevant problems such as mechanical stability of liquid bridges between non-flat geometries such as spheres or fibers.

Chapter 6 offers a direct experimental evidence of the theoretically predicted hydrodynamic instability of 1D ionic conduction [46, 117]. The related critical onset of the flow should be clearly distinguished from that in a multidimensional setting (e.g., at a curved interface corresponding to a spherical ion exchange granule [108, 109, 110] or undulated membrane surface [116], conductively heterogeneous membrane surface [125] or array of nanochannels [113]). In these cases any kind of EO initiates a flow in a thresholdless manner, that is, right upon application of an electric field. Hence, while qualitatively nonclassical EO may be recognized by its higher velocities, the precise type of the slip can only be identified by studying the dependence of velocity on the electric field. Thus, a linear dependence is expected in the classical case of quasiequilibrium EO at an originally charged interface [126] and a quadratic dependence for quasiequilibrium induced charge EO at an originally noncharged interface [119, 120, 121]. On the other hand, for the extended charge EO, cubic field dependence is expected, switching at higher voltage to a quadratic one with the tangential current density variation as a driving factor instead of the electric field [46, 117]. Namely, this type of flow has been reported recently at an array of nanochannels [113. Likely, many more reported flows, attributed in the past to other mechanisms, belonged in fact to this category [11, 112 . 


\section{SUMMARY AND OUTLOOK}

\subsection{Outlook}

In this thesis we describe electrowetting as a tool to manipulate liquid interfaces. In particular we showed that electrowetting can be used to achieve reversible transitions between Cassie-Baxter and Wenzel states. Applied electric field not only affects the equilibrium shape of a liquid interface, it also gives rise to electrokinetic effects of fluids near solid walls.

Chapter 2 of provides understanding of the microscopic mechanism controlling the properties of the superhydrophobic state under electrowetting conditions and in particular the stability limit of the Cassie-Baxter state. Such a detailed understanding is crucial to reach the holy grail of reversible switching between these states. Electrowetting induced reversible transitions have several advantages compared to other approaches (partial evaporation [33], violent mechanical shaking [60] etc.). It offers opportunities for fast and precise fine-tuning of the wetting state. Based on our findings presented in Chapter 2, in Chapter 3 we present two approaches for achieving reversibility. In particular a system with patterned electrodes has great potential as a simple, fast in operation (the transition time from the Wenzel to the Cassie-Baxter state is $5-10 \mathrm{~ms}$ ) technique for reversible switching between Cassie-Baxter and Wenzel states. It is also easy to implement in microfluidic or Lab-on-a-Chip systems. However, more research is needed to determine the optimal set of parameters such as width of the electrodes, the distance between them, etc.. Our results also open up prospective

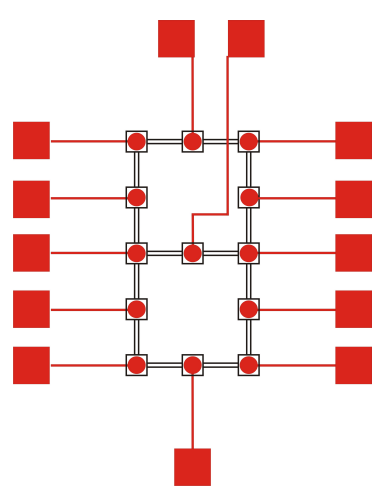

Figure 7.1: Sketch of a single cell of an electrowetting induced microscreen. The red color represents transparent electrode. 
for development of several optoelectronic devices: an electrowetting induced segmented display for instance, as sketched in Fig.7.1. Here we use the fact that at Cassie-Baxter state the meniscus reflects the light appearing bright, whereas at the Wenzel state it turns dark. Thus, by turning on or off individually addressable segments, it is possible to produce simplified representations of the Arabic numerals or letters in small scales. The characteristic size of the segments in this device may reach down to $10 X 10 \mu m$.

If in experiments presented in Chapter 4 the ambient air is replaced by non transparent oil the system can be used as an elecrowetting driven optical switch with tunable aperture. This idea was developed by our colleagues [127]. Fig. 7.2 shows a schematic of the optical switch.
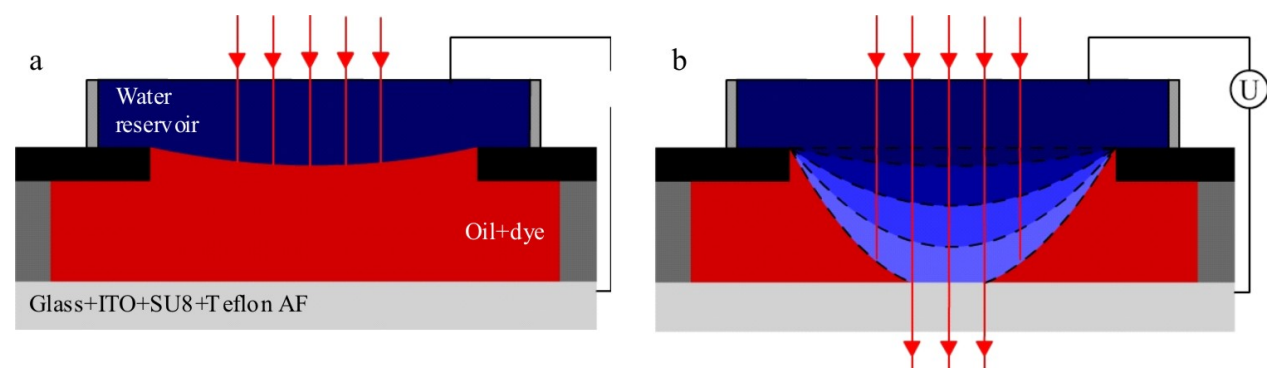

Figure 7.2: Schematic side view of the electrowetting driven optical switch and aperture.

When a voltage is applied between the water phase and the lower substrate (electrode), an electric field is generated within the oil phase. Due to the resulting electric stresses the water-oil interface is deflected downwards. As the voltage increases, the deflection increases until it reaches a critical threshold. This threshold value depends on the aspect ratio between the radius of the hole and the height between the glass plates as well as the physical properties of the fluids []. Once the threshold is reached, the meniscus becomes unstable and abruptly snaps down to the lower substrate, where it creates a transparent spot (Fig. out1 b). The dynamics of this process are governed by the balance of Maxwell stress, surface tension and viscous stresses. When the voltage is turned off the water/oil interface returns back to its original position since the presence of oil provides low 


\section{SUMMARY AND OUTLOOK}

hysteresis. The resulting optical switch can be tuned/operated by applying a DC voltage, an $\mathrm{AC}$ voltage at $1 \mathrm{kHz}$ (or higher) or by applying amplitude modulation.

The optical switch can attain $95 \%$ intensity attenuation and has a response time of $\sim 2$ and $\sim 120 \mathrm{~ms}$ for switching on and off, respectively.

The same system can be used also as an optical aperture. Its diameter is controlled by the modulation frequency and maximum amplitude of the applied voltage. Moreover, the optical switches with tunable aperture as presented here have great potential in optical communication and in display devices, because they can be arranged in an array of individually addressable apertures. In Fig. 7.3 we demonstrate this potential by presenting a device consisting of two optical switches/apertures, positioned close to each other, that can be addressed independently without any cross talk.
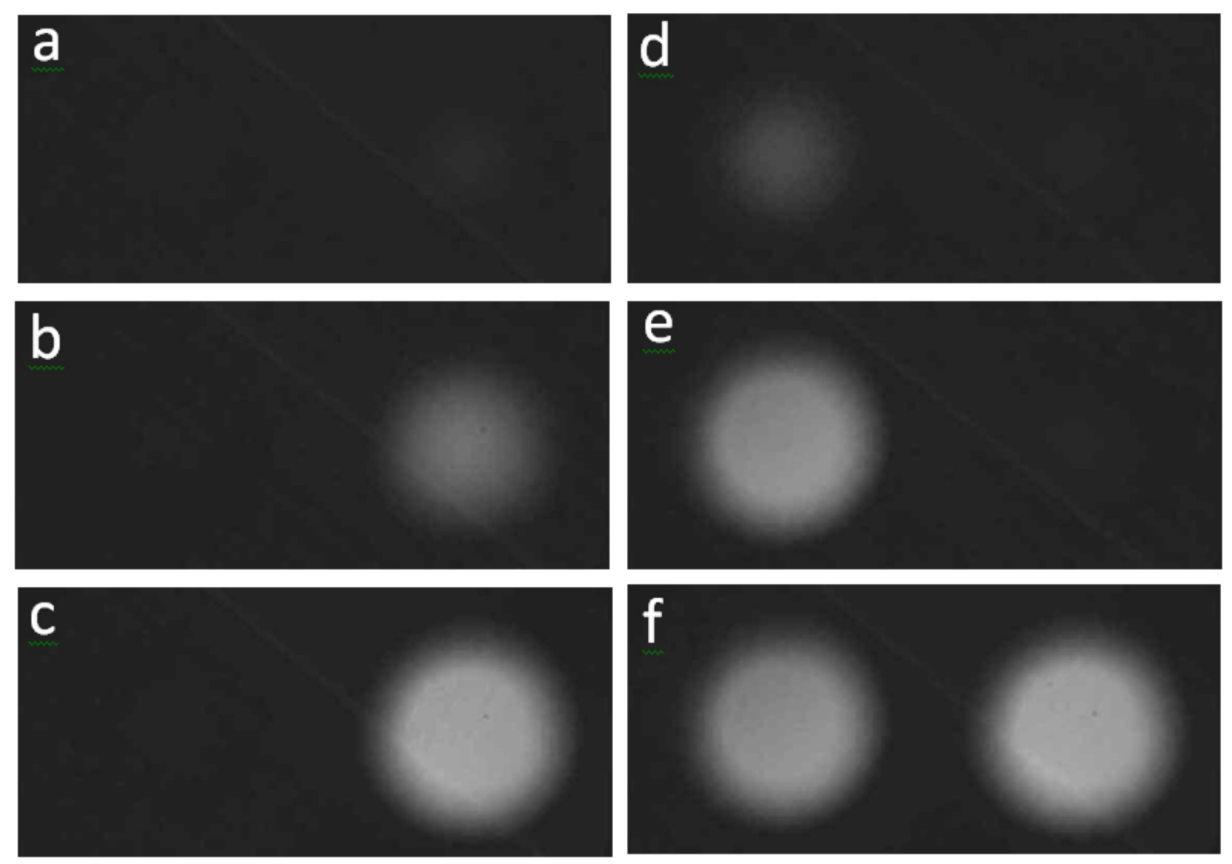

Figure 7.3: Two optical switches, positioned close together, can be addressed independently. .

In Chapter 5, we validated electrowetting as a tool to study wetting problems of non-flat geometries. These findings open alleys to explore industrially 
relevant problems such as mechanical stability of liquid bridges between non-flat geometries such as spheres or fibers. I believe that these results may open up possibilities to further extend the notion of utilizing wettability to synthesize complex colloidal systems.

Experimental results of Chapter 6 suggest an accurate quantitative experimental study of the vicinity of the instability threshold as a natural continuation of the current preliminary qualitative report. Another natural follow up should concern the study of the dependence of the physical parameters of the system (ionic valencies and ionic 'viscous' radii, dielectric permeability, ionic diffusivities ratio, etc) on instability threshold and nonlinear flow characteristics affecting the crucial dimensionless combinations in the system, such as the Peclet number and the dimensionless Debye length in the system. 
7. SUMMARY AND OUTLOOK 


\section{References}

[1] M. W. J. Prins, W. J. J. Welters, and J. W. Weekamp. Fluid control in multichannel structures by electrocapillary pressure. Science, 291(5502):277280, 2001. 1, 10

[2] T. B. Jones, K.-L. Wang, and D.-J. Yao. Frequency-dependent electromechanics of aqueous liquids: electrowetting and dielectrophoresis. Langmuir, 20(7):2813-2818, 2004. PMID: 15835158. 1, 10

[3] Chester T. O'Konski and Henry C. Thacher. The distortion of aerosol droplets by an electric field. The Journal of Physical Chemistry, 57(9):955958, 1953. 2, 10

[4] A. Bateni, S. S. Susnar, A. Amirfazli, and A. W. Neumann. Development of a new methodology to study drop shape and surface tension in electric fields. Langmuir, 20(18):7589-7597, 2004. PMID: 15323506. 2, 10

[5] Brian G. Prevo Orlin, D. Velev and H. Bhatt Ketan. On-chip manipulation of free droplets. Nature, 426:515-516, 2003. 2, 10

[6] W. Barthlott and C. Neinhuis. Purity of the sacred lotus, or escape from contamination in biological surfaces. Planta, 202:1-8, 1997. 10.1007/s004250050096. 5

[7] Lichao Gao and Thomas J. McCarthy. The lotus effect explained: two reasons why two length scales of topography are important. Langmuir, 22(7):2966-2967, 2006. PMID: 16548542. 4 


\section{REFERENCES}

[8] T. Nakanishi, T. Michinobu, K. Yoshida, N. Shirahata, K. Ariga, H. Mhwald, and D.G. Kurth. Nanocarbon superhydrophobic surfaces created from fullerene-based hierarchical supramolecular assemblies. Advanced Materials, 20(3):443-446, 2008. 4

[9] Hye-Mi Bok, Tae-Yeon Shin, and Sungho Park. Designer binary nanostructures toward water slipping superhydrophobic surfaces. Chemistry of Materials, 20(6):2247-2251, 2008. 4

[10] Barbara Cortese, Stefania D'Amone, Michele Manca, Ilenia Viola, Roberto Cingolani, and Giuseppe Gigli. Superhydrophobicity due to the hierarchical scale roughness of pdms surfaces. Langmuir, 24(6):2712-2718, 2008. PMID: 18217778. 4

[11] Panagiotis N. Manoudis, Ioannis Karapanagiotis, Andreas Tsakalof, Ioannis Zuburtikudis, and Costas Panayiotou. Superhydrophobic composite films produced on various substrates. Langmuir, 24(19):11225-11232, 2008. PMID: 18720965. 4

[12] N.J. Shirtcliffe, G. McHale, M.I. Newton, G. Chabrol, and C.C. Perry. Dual-scale roughness produces unusually water-repellent surfaces. Advanced Materials, 16(21):1929-1932, 2004. 4. 34

[13] Wei Chen, Alexander Y. Fadeev, Meng Che Hsieh, Didem ner, Jeffrey Youngblood, and Thomas J. McCarthy. Ultrahydrophobic and ultralyophobic surfaces: some comments and examples. Langmuir, 15(10):3395-3399, 1999. 4

[14] R.N. Wenzel. RESISTANCE OF SOLID SURFACES TO WETTING BY WATER. Industrial \& Engineering Chemistry, 28(8):988-994, 1936. 5. 8

[15] AB.D. Cassie and S. Baxter. Wettability of porous surfaces. Trans. Faraday Soc, 40:546-551, 1944. 5

[16] C. Yang, U. Tartaglino, and B. N. J. Persson. Influence of surface roughness on superhydrophobicity. Phys. Rev. Lett., 97(11):116103, Sep 2006. 5 
[17] Mathilde Callies and David Quere. On water repellency. Soft Matter, 1:5561, 2005. 6. 59

[18] DETTRE ROBERT H. and JOHNSON RULON E. Contact Angle Hysteresis, chapter 9, pages 136-144. 6

[19] Michael Nosonovsky. Multiscale roughness and stability of superhydrophobic biomimetic interfaces. Langmuir, 23(6):3157-3161, 2007. 6

[20] H. Kusumaatmaja, M. L. Blow, A. Dupuis, and J. M. Yeomans. The collapse transition on superhydrophobic surfaces. EPL (Europhysics Letters), 81(3):36003, 2008. 9

[21] C. Ishino, K. Okumura, and D. Qur. Wetting transitions on rough surfaces. EPL (Europhysics Letters), 68(3):419, 2004. 9

[22] Junghoon Lee, Bo He, and Neelesh A Patankar. A roughness-based wettability switching membrane device for hydrophobic surfaces. Journal of Micromechanics and Microengineering, 15(3):591, 2005. 9, 34

[23] Ting-Hsuan Chen, Yun-Ju Chuang, Ching-Chang Chieng, and Fan-Gang Tseng. A wettability switchable surface by microscale surface morphology change. Journal of Micromechanics and Microengineering, 17(3):489, 2007. 9. 34

[24] Jonathan B. Boreyko and Chuan-Hua Chen. Restoring superhydrophobicity of lotus leaves with vibration-induced dewetting. Phys. Rev. Lett., 103(17):174502, Oct 2009. 9, 35

[25] M. Jin, X. Feng, L. Feng, T. Sun, J. Zhai, T. Li, and L. Jiang. Superhydrophobic aligned polystyrene nanotube films with high adhesive force. Advanced Materials, 17(16):1977-1981, 2005. 9, 34

[26] Xia Hong, Xuefeng Gao, and Lei Jiang. Application of superhydrophobic surface with high adhesive force in no lost transport of superparamagnetic microdroplet. Journal of the American Chemical Society, 129(6):1478-1479, 2007. 9,34 


\section{REFERENCES}

[27] Feng Zhou and Wilhelm T. S. Huck. Three-stage switching of surface wetting using phosphate-bearing polymer brushes. Chem. Commun., pages 5999-6001, 2005. 9, 34

[28] Taolei Sun, Guojie Wang, Lin Feng, Biqian Liu, Yongmei Ma, Lei Jiang, and Daoben Zhu. Reversible switching between superhydrophilicity and superhydrophobicity. Angewandte Chemie International Edition, 43(3):357-360, 2004. 9, 34

[29] Qiang Fu, G. V. Rama Rao, Solomon B. Basame, David J. Keller, Kateryna Artyushkova, Julia E. Fulghum, and Gabriel P. Lpez. Reversible control of free energy and topography of nanostructured surfaces. Journal of the American Chemical Society, 126(29):8904-8905, 2004. 9, 34

[30] Athanassia Athanassiou, Maria I. Lygeraki, Dario Pisignano, Kleanthi Lakiotaki, Maria Varda, Elisa Mele, Costas Fotakis, Roberto Cingolani, and Spiros H. Anastasiadis. Photocontrolled variations in the wetting capability of photochromic polymers enhanced by surface nanostructuring. Langmuir, 22(5):2329-2333, 2006. 9, 34

[31] Ho Sun Lim, Joong Tark Han, Donghoon Kwak, Meihua Jin, and Kilwon Cho. Photoreversibly switchable superhydrophobic surface with erasable and rewritable pattern. Journal of the American Chemical Society, 128(45):14458-14459, 2006. 9, 34

[32] Xinjian Feng, Lin Feng, Meihua Jin, Jin Zhai, Lei Jiang, and Daoben Zhu. Reversible super-hydrophobicity to super-hydrophilicity transition of aligned zno nanorod films. Journal of the American Chemical Society, 126(1):62-63, 2004. 9, 34

[33] Tom N. Krupenkin, J. Ashley Taylor, Evelyn N. Wang, Paul Kolodner, Marc Hodes, and Todd R. Salamon. Reversible wetting-dewetting transitions on electrically tunable superhydrophobic nanostructured surfaces. Langmuir, 23(18):9128-9133, 2007. PMID: 17663572. 9, 20, 21, 34 
[34] M.S. Dhindsa, N.R. Smith, J. Heikenfeld, P.D. Rack, J.D. Fowlkes, M.J. Doktycz, A.V. Melechko, and M.L. Simpson. Reversible electrowetting of vertically aligned superhydrophobic carbon nanofibers. Langmuir, 22(21):9030-9034, 2006. 9, 10, 20, 34

[35] T. Krupenkin, J.A. Taylor, T.M. Schneider, and S. Yang. From Rolling Ball to Complete Wetting: The Dynamic Tuning of Liquids on Nanostructured Surfaces. Langmuir, 20:3824-3827, 2004. 9, 20, 21, 34

[36] D.L. Herbertson, C.R. Evans, N.J. Shirtcliffe, G. McHale, and M.I. Newton. Electrowetting on superhydrophobic SU-8 patterned surfaces. Sensors and actuators. A, Physical, 130:189-193, 2006. 10, 20, 21.

[37] Sung Kwon Cho, Hyejin Moon, and Chang-Jin Kim. Creating, transporting, cutting, and merging liquid droplets by electrowetting-based actuation for digital microfluidic circuits. Microelectromechanical Systems, Journal of, 12(1):70 - 80, feb 2003. 10

[38] Marguerite Bienia, Catherine Quilliet, and Marcel Vallade. Modification of drop shape controlled by electrowetting. Langmuir, 19(22):9328-9333, 2003. 10

[39] Anke Klingner, Stephan Herminghaus, and Frieder Mugele. Self-excited oscillatory dynamics of capillary bridges in electric fields. Applied Physics Letters, 82(23):4187-4189, 2003. 10

[40] F. Mugele and J.C. Baret. Electrowetting: from basics to applications. J. Phys.: Condens. Matter, 17(28):705-744, 2005. 11, 26, 29, 57, 63

[41] Catherine Quilliet and Bruno Berge. Electrowetting: a recent outbreak. Current Opinion in Colloid \& Interface Science, 6(1):34 - 39, 2001. 11

[42] Thomas B. Jones. On the relationship of dielectrophoresis and electrowetting. Langmuir, 18(11):4437-4443, 2002. 12, 64

[43] Jun Zeng and Tom Korsmeyer. Principles of droplet electrohydrodynamics for lab-on-a-chip. Lab Chip, 4:265-277, 2004. 12 


\section{REFERENCES}

[44] W. V. Van Roosbroeck. Theory of flow of electrons and holes in germanium and other semiconductors. Bell System Tech. J., 29(1):560, 1950. 13

[45] V. G. Levichf. Physicochemical Hydrodynamics. Prentice-Hall, Englewood Cliffs, NJ, 1962. 14, 15, 72

[46] I. Rubinstein and B. Zaltzman. Electro-osmotically induced convection at a permselective membrane. Phys. Rev. E, 62(2):2238-2251, Aug 2000. 16 , 17, $72,78,79,80,83$

[47] Irina Lerman, Isaak Rubinstein, and Boris Zaltzman. Absence of bulk electroconvective instability in concentration polarization. Phys. Rev. E, 71(1):011506, Jan 2005. 17

[48] Isaak Rubinstein, Boris Zaltzman, and Irina Lerman. Electroconvective instability in concentration polarization and nonequilibrium electro-osmotic slip. Phys. Rev. E, 72(1):011505, Jul 2005. 17

[49] G. Manukyan, J. M. Oh, D. van den Ende, R. G. H. Lammertink, and F. Mugele. Electrical switching of wetting states on superhydrophobic surfaces: A route towards reversible cassie-to-wenzel transitions. Phys. Rev. Lett., 106(1):014501, Jan 2011. 19, 33

[50] D. Quéré. Non-sticking drops. Reports on Progress in Physics, 68(11):24952532, 2005. 20, 22, 44

[51] Chang-Hwan Choi and Chang-Jin Kim. Large slip of aqueous liquid flow over a nanoengineered superhydrophobic surface. Phys. Rev. Lett., 96(6):066001, Feb 2006. 20

[52] P. Joseph, C. Cottin-Bizonne, J.-M. Benoît, C. Ybert, C. Journet, P. Tabeling, and L. Bocquet. Slippage of water past superhydrophobic carbon nanotube forests in microchannels. Phys. Rev. Lett., 97(15):156104, Oct 2006. 20

[53] Audrey Steinberger, Cecile Cottin-Bizonne, Pascal Kleimann, and Elisabeth Charlaix Journal. High friction on a bubble mattress. Nat Mater, 6(9):665-668, Jan 2007. 20 
[54] Helmut Rathgen, Kazuyasu Sugiyama, Claus-Dieter Ohl, Detlef Lohse, and Frieder Mugele. Nanometer-resolved collective micromeniscus oscillations through optical diffraction. Phys. Rev. Lett., 99(21):214501, Nov 2007. 20

[55] Helmut Rathgen and Frieder Mugele. Microscopic shape and contact angle measurement at a superhydrophobic surface. Faraday Discuss., 146:49-56, 2010. 20, 29, 31, 81 ,

[56] Nicolas Verplanck, Yannick Coffinier, Vincent Thomy, and Rabah Boukherroub. Wettability switching techniques on superhydrophobic surfaces. Nanoscale Research Letters, 2:577-596, 2007. 10.1007/s11671-007-9102-4. 20

[57] N. Verplanck, E. Galopin, J.C. Camart, V. Thomy, Y. Coffinier, and R. Boukherroub. Reversible Electrowetting on Superhydrophobic Silicon Nanowires. Nano Lett, 7(3):813-817, 2007. 20

[58] A. Ahuja, J. A. Taylor, V. Lifton, A. A. Sidorenko, T. R. Salamon, E. J. Lobaton, P. Kolodner, and T. N. Krupenkin. Nanonails: A simple geometrical approach to electrically tunable superlyophobic surfaces. Langmuir, 24(1):9-14, 2008. PMID: 17929955. 20, 21

[59] Vaibhav Bahadur and Suresh V. Garimella. Electrowetting-based control of static droplet states on rough surfaces. Langmuir, 23(9):4918-4924, 2007. 20

[60] Jonathan B. Boreyko and Chuan-Hua Chen. Restoring superhydrophobicity of lotus leaves with vibration-induced dewetting. Phys. Rev. Lett., 103(17):174502, Oct 2009. 20

[61] G Wiegand, KR Neumaier, and E Sackmann. Microinterferometry: threedimensional reconstruction of surface microtopography for thin-film and wetting studies by reflection interference contrast microscopy (ricm). Applied Optics, 37(29):6892-6905, Jan 1998. 25 


\section{REFERENCES}

[62] Juergen Buehrle, Stephan Herminghaus, and Frieder Mugele. Interface profiles near three-phase contact lines in electric fields. Phys. Rev. Lett., 91(8):086101, Aug 2003. 26. 58

[63] M. Reyssat, J. M. Yeomans, and D. Quéré. Impalement of fakir drops. EPL (Europhysics Letters), 81(2):26006, 2008. 29, 44, 49, 53, 82

[64] Oh, J. M., Manukyan, G., Ende, D. van den, and Mugele, F. Electric-fielddriven instabilities on superhydrophobic surfaces. EPL, 93(5):56001, 2011. $29,43,46,48$

[65] M. Sbragaglia, A.M. Peters, C. Pirat, B.M. Borkent, R.G.H. Lammertink, M. Wessling, and D. Lohse. Spontaneous Breakdown of Superhydrophobicity. Phys. Rev. Lett., 99(15):156001, 2007. 30, 31, 44

[66] A. Steinberger, C. Cottin-Bizonne, P. Kleimann, and E. Charlaix. Nanoscale flow on a bubble mattress: Effect of surface elasticity. Phys. Rev. Lett., 100(13):134501, Mar 2008. 31, 81

[67] Sung Hee Ko, Horim Lee, and Kwan Hyoung Kang. Hydrodynamic flows in electrowetting. Langmuir, 24(3):1094-1101, 2008. PMID: 18177057. 35

[68] F. Mugele, J.C. Baret, and D. Steinhauser. Microfluidic mixing through electrowetting-induced droplet oscillations. Applied Physics Letters, 88(20):204106-204106, 2006. 35

[69] Aurelie Lafuma and David Quere. Superhydrophobic states. Nature Materials, 2(7):457-460, 2003. 44

[70] Neelesh A. Patankar. Mimicking the lotus effect: influence of double roughness structures and slender pillars. Langmuir, 20(19):8209-8213, 2004. PMID: 15350093. 44

[71] Neelesh A. Patankar. Hysteresis with regard to cassie and wenzel states on superhydrophobic surfaces. Langmuir, 26(10):7498-7503, 2010. 44, 49

[72] Abraham Marmur. The lotus effect: superhydrophobicity and metastability. Langmuir, 20(9):3517-3519, 2004. PMID: 15875376. 44 
[73] Helmut Rathgen and Frieder Mugele. Microscopic shape and contact angle measurement at a superhydrophobic surface. Faraday Discuss., 146:49-56, 2010. 44, 53, 82

[74] Geoffrey Taylor. Disintegration of water drops in an electric field. Proceedings of the Royal Society of London. Series A, Mathematical and Physical Sciences, 280(1382):pp. 383-397, 1964. 50

[75] A. Jaworek and A.T. Sobczyk. Electrospraying route to nanotechnology: An overview. Journal of Electrostatics, 66(3-4):197 - 219, 2008. 50

[76] H. B. Eral, G. Manukyan, and J. M. Oh. Wetting of a drop on a sphere. Langmuir, 27(9):5340-5346, 2011. 55

[77] P.G. de Gennes, F. Brochard-Wyart, and D. Quere. Capillarity and wetting phenomena: Drops, bubbles, pearls, waves. Springer, New York, 2004. 56

[78] J Plateau. Experimental and theoretical statics of liquids subject to molecular forces only. (Translated from French.). Gauthier-Villars, Paris, 1873. 56

[79] Daniela J. Kraft, Wessel S. Vlug, Carlos M. van Kats, Alfons van Blaaderen, Arnout Imhof, and Willem K. Kegel. Self-assembly of colloids with liquid protrusions. Journal of the American Chemical Society, 131(3):1182-1186, 2009. 56

[80] Eric B. Mock, Hank De Bruyn, Brian S. Hawkett, Robert G. Gilbert, and Charles F. Zukoski. Synthesis of anisotropic nanoparticles by seeded emulsion polymerization. Langmuir, 22(9):4037-4043, 2006. 56

[81] H. B. Eral, D. van den Ende, F. Mugele, and M. H. G. Duits. Influence of confinement by smooth and rough walls on particle dynamics in dense hard-sphere suspensions. Phys. Rev. E, 80(6):061403, Dec 2009. 56

[82] D Psaltis, S.R Quake, and C Yang. Developing optofluidic technology through the fusion of microfluidics and optics. Nature, 442(7101):381, 2006. 56 


\section{REFERENCES}

[83] Sung Kwon Cho, Hyejin Moon, and Chang-Jin Kim;. Creating, transporting, cutting, and merging liquid droplets by electrowetting-based actuation for digital microfluidic circuits. JMEMS, 12(1):70-80, 2003. 56

[84] B. M. Mognetti and J. M. Yeomans. Capillary filling in microchannels patterned by posts. Phys. Rev. E, 80(5):056309, Nov 2009. 56

[85] B. M. Mognetti and J. M. Yeomans. Using electrowetting to control interface motion in patterned microchannels. Soft Matter, 6:2400-2402, 2010. 56

[86] H. B. Eral, J. M. Oh, D. van den Ende, F. Mugele, and M. H. G. Duits. Anisotropic and hindered diffusion of colloidal particles in a closed cylinder. Langmuir, 26(22):16722-16729, 2010. 56

[87] B.J. Carroll. Colloids and Surfaces A, 74(2-3):131 - 167, 1993. 56, 68

[88] J Heikenfeld, K Zhou, E. Kreit, B. Raj, S. Yang, B. Sun, A. Milarcik, L. Clapp, and R. Schwartz. Electrofluidic displays using young-laplace transposition of brilliant pigment dispersions. Nature Photonics, 3:292-296, 2009. 56

[89] K Zhou and J Heikenfeld. Arrayed electrowetting microwells. Applied Physics Letters, 92(11):113515, 2008. 56

[90] J Chen. Electrowetting-actuated zoom lens with spherical-interface liquid lenses. Journal of the Optical Society of America A, 25(11):2644, 2008. 56

[91] R. Hołyst and A. Poniewierski. Wetting on a spherical surface. Phys. Rev. $B, 36(10): 5628-5630$, Oct 1987. 56

[92] P. J. Upton, J. O. Indekeu, and J. M. Yeomans. Wetting on spherical and cylindrical substrates: Global phase diagrams. Phys. Rev. B, 40(1):666-679, Jul 1989. 56

[93] Ioannis A. Hadjiagapiou. Wetting on a spherical-shell substrate. J. Phys. Chem. B, 101(44):8990-8996, 1997. 56 
[94] M. C. Stewart and R. Evans. Wetting and drying at a curved substrate: Long-ranged forces. Phys. Rev. E, 71(1):011602, Jan 2005. 56

[95] C. W. Extrand and S. I. Moon. Contact angles on spherical surfaces. Langmuir, 24(17):9470, 2008. 56

[96] G. Mason and W.C. Clark. Liquid bridges between spheres. Chemical Engineering Science, 20(10):859 - 866, 1965. 56

[97] F. Mugele and J. Buehrle. Equilibrium drop surface profiles in electric fields. Journal of Physics: Condensed Matter, 19(37):375112, 2007. 58

[98] Stephan Herminghaus, Martin Brinkmann, and Ralf Seemann. Wetting and dewetting of complex surface geometries. Annual Review of Materials Research, 38(1):101-121, 2008. 59

[99] Bram M. Borkent, Sissi de Beer, Frieder Mugele, and Detlef Lohse. On the shape of surface nanobubbles. Langmuir, 26(1):260-268, 2010. 59

[100] Martin Brinkmann and Reinhard Lipowsky. Wetting morphologies on substrates with striped surface domains. Journal of Applied Physics, 92(8):4296-4306, 2002. 59

[101] Jean-Christophe Baret, Michel Decra, Stephan Herminghaus, and Ralf Seemann. Electroactuation of fluid using topographical wetting transitions. Langmuir, 21(26):12218-12221, 2005. 59

[102] T. B. Jones, Jesse David Fowler, Young Soo Chang, and Chang-Jin Kim. Frequency-based relationship of electrowetting and dielectrophoretic liquid microactuation. Langmuir, 19(18):7646-7651, 2003. 64

[103] Kwan Hyoung Kang. How electrostatic fields change contact angle in electrowetting. Langmuir, 18(26):10318-10322, 2002. 64

[104] H.B. Eral, D. Ende van den, F. Mugele, and M.H.G. Duits. Influence of confinement by smooth and rough walls on particle dynamics in dense hard-sphere suspensions. Phys. Rev. E, 80(6):061403, 2009. 68 


\section{REFERENCES}

[105] B.J. Carroll. Langmuir, 2(2):248-250, 1986. 68

[106] S. M. Rubinstein, G. Manukyan, A. Staicu, I. Rubinstein, B. Zaltzman, R. G. H. Lammertink, F. Mugele, and M. Wessling. Direct observation of a nonequilibrium electro-osmotic instability. Phys. Rev. Lett., 101(23):236101, Dec 2008. 71

[107] Yilong Han and David G. Grier. Colloidal electroconvection in a thin horizontal cell. ii. bulk electroconvection of water during parallel-plate electrolysis. The Journal of Chemical Physics, 125(14):144707, 2006. 72, 73

[108] N.A. Mishchuk S.S. Dukhin and P.B. Takhistov. Coll. J. USSR, (in Russ.), 51(616), 1989. 72, 73, 80, 83

[109] Felix C. Leinweber and Ulrich Tallarek. Nonequilibrium electrokinetic effects in beds of ion-permselective particles. Langmuir, 20(26):11637-11648, 2004. PMID: 15595793. 72, 73, 80, 83

[110] Y. BEN and H.-C. CHANG. Nonlinear smoluchowski slip velocity and micro-vortex generation. Journal of Fluid Mechanics, 461:229-238, 2002. $72,73,80,83$

[111] S. Reich, B. Gavish, and S. Lifson. Visualization of hydrodynamic phenomena in the vicinity of a semipermeable membrane. Desalination, 24(1-3):295 $-296,1977.72,80,83$

[112] Shneior Lifson, Benjamin Gavish, and Shimon Reich. Flicker noise of ionselective membranes and turbulent convection in the depleted layer. $E u$ ropean Biophysics Journal, 4:53-65, 1978. 10.1007/BF00538840. 72, 80. 83

[113] Sung Jae Kim, Ying-Chih Wang, Jeong Hoon Lee, Hongchul Jang, and Jongyoon Han. Concentration polarization and nonlinear electrokinetic flow near a nanofluidic channel. Phys. Rev. Lett., 99(4):044501, Jul 2007. 72. 80,83 
[114] Rosler H.-W. Staude E. Maletzki, F. Ion transfer across electrodialysis membranes in the overlimiting current range: Stationary voltage current characteristics and current noise power spectra under different conditions of free convection. Journal of Membrane Science, 71(1-2):105-115, 1992. cited By (since 1996) 44. 72, 73, 75

[115] B. Pretz J. Rubinshtein, I. Zaltzman and C. Linder. Experimental verification of the electroosmotic mechanism of overlimiting conductance through a cation exchange electrodialysis membrane. Russian Journal of Electrochemistry, 38:853-863, 2002. 10.1023/A:1016861711744. 72, 73, 75

[116] J. Balster, M. H. Yildirim, D. F. Stamatialis, R. Ibanez, R. G. H. Lammertink, V. Jordan, and M. Wessling. Morphology and microtopology of cation-exchange polymers and the origin of the overlimiting current. The Journal of Physical Chemistry B, 111(9):2152-2165, 2007. 72, 80, 83

[117] B. ZALTZMAN and I. RUBINSTEIN. Electro-osmotic slip and electroconvective instability. Journal of Fluid Mechanics, 579:173-226, 2007. 72, 78, $79,80,83$

[118] S.S. Dukhin and B.V. Derjaguin. Electrophoresis (in Russ.). Nauka, Moscow, 1976. 72

[119] Martin Z. Bazant and Todd M. Squires. Induced-charge electrokinetic phenomena: Theory and microfluidic applications. Phys. Rev. Lett., 92(6):066101, Feb 2004. 72, 80, 83

[120] TODD M. SQUIRES and MARTIN Z. BAZANT. Induced-charge electroosmosis. Journal of Fluid Mechanics, 509:217-252, 2004. 72, 80, 83

[121] Katsuyo Bazant, Martin Z. Thornton and Armand Ajdari. Diffuse-charge dynamics in electrochemical systems. Phys. Rev. E, 70(2):021506, Aug 2004. 72, 80, 83,

[122] S.S. Dukhin. Electrokinetic phenomena of the second kind and their applications. Advances in Colloid and Interface Science, 35:173 - 196, 1991. 72 


\section{REFERENCES}

[123] N. Mishchuk, F., Gonzalez-Caballero, and P. Takhistov. Electroosmosis of the second kind and current through curved interface. Colloids and Surfaces A: Physicochemical and Engineering Aspects, 181(1-3):131 - 144, 2001. 72

[124] I. Rubinstein, E. Staude, and O. Kedem. Role of the membrane surface in concentration polarization at ion-exchange membrane. Desalination, $69(2): 101-114,1988.72,73,75$

[125] R. Ibanez, D. F. Stamatialis, and M. Wessling. Role of membrane surface in concentration polarization at cation exchange membranes. Journal of Membrane Science, 239(1):119 - 128, 2004. Special Issue in Honor of Dr. Ora Kedem. 80, 83

[126] R.F. Probstein. Physicochemical Hydrodynamics : An Introduction. Butterworths, Boston, 1989. 80, 83

[127] C. U. Murade, J. M. Oh, D. van den Ende, and F. Mugele. Electrowetting driven optical switch and tunable aperture. Opt. Express, 19(16):1552515531, Aug 2011. 85 


\section{Samenvatting}

Het hoofdthema van dit proefschrift is de elektrische manipulatie van vloeistoffen aan grensvlakken. Dit proefschrift presenteert zowel experimenteel als numeriek onderzoek aan de toepassing van electrowetting op superhydrofobe oppervlakken. In het bijzonder tonen onze resultaten de weg naar omkeerbare omschakelingen op superhydrofobe oppervlakken. Enkele aspecten van elektroconvectie worden ook beschreven in dit werk.

De resultaten beschreven in Hoofdstuk 2 tonen de mate van flexibiliteit en beheersing die worden verkregen over de superhydrofobiciteit bij gebruik van een elektrisch veld. Bij lage spanningen zorgt de omkeerbare buiging van de micromenisci voor een precieze afstemming van de hydrodynamische slip [1] en de optische diffractie van deze oppervlakken [2]. We hebben duidelijk aangetoond dat het loslaten van de contactlijnen het kritieke voltage bepaalt voor de onomkeerbare overgang naar de Wenzel toestand. In combinatie met de voorwaarde voor co-existentie van de Cassie-Baxter en Wenzel toestanden, laten we in Hoofdstuk 3 een gelokaliseerde en omkeerbare omschakeling tussen de twee bevochtingstoestanden zien. Deze gelokaliseerde, omkeerbare omschakeling vereist een meer systematisch onderzoek. We hebben ook laten zien dat oscillaties in de druppelvorm ten gevolge van een laagfrequent ac elektrisch veld $(\sim 100 H z)$ ook een overgang van de gedeeltelijke Wenzel toestand naar de Cassie-Baxter toestand kunnen veroorzaken.

Ons numeriek en experimenteel onderzoek in Hoofdstuk 4 laat zien dat elektrohydrodynamische instabiliteiten een belangrijke limiterende factor zijn voor de stabiliteit van de Cassie-Baxter toestand op superhy- 
drofobe oppervlakken onder invloed van een elektrisch veld. Elekrohydrodynamische instabiliteiten wedijveren met de klassieke overgangsvoorwaarde (het loslaten van de contactlijn aan de randen van de oppervlaktetopografie), welke de overhand heeft bij kleine contacthoeken. Wanneer de diepte-breedte verhouding van de oppervlaktetopografie groter is dan 2, wordt de kritieke "Taylor kegel"-achtige vorm van het oppervlak onafhankelijk van de diepte-breedte verhouding. Dit betekent dat er een constante kritische contacthoek is die het regime van het loslaten van de contactlijn en elektrohydrodynamische instabiliteiten van elkaar scheidt. Voor superhydrofobe oppervlakken met geometrisch overhangende delen, welke het loslaten van de contactlijn onderdrukken, is het aannemelijk dat elektrohydrodynamische instabiliteiten het dominante instabiliteitmechanisme zijn. We laten ook zien dat het vloeistof-gas grensvlak onder invloed van een toegepast elektrisch veld niet bolvormig is. De reden hiervoor is een niethomogene verdeling van de elektrische krachten langs het grensvlak. Dit leidt tot een niet-bolvormige vervorming van het grensvlak, in tegenstelling tot een druk-gedreven overgang op traditionele superhydrofobe oppervlakken [3, 4], waar de vorm van het grensvlak enkel wordt bepaald door de Laplace druk.

In hoofdstuk 5 valideren we eerst electrowetting als een methode om bevochtiging van niet-vlakke geometrien te bestuderen. Vertouwend op deze geldigheid, bestuderen we vervolgens - zowel experimenteel als analytisch - de evenwichtstoestanden van een klassieke bevochtigingsgeometrie, namelijk een druppel op een bol, als functie van de beschrijvende variabelen, het druppelvolume en de contacthoek.

Als validatie-experiment hebben we systematisch de invloed van substraat geometrie op het electrowettinggedrag bestudeerd, met het oog op het effect van druppelvolume, en de daaruit volgende relatieve kromming. Het electrowettinggedrag is gelijk voor verschillende druppelvolumes, wat overeenkomt met verschillende krommingen van de druppel relatief tot het substraat. Dit laat zien dat 
krommingseffecten verwaarloosd kunnen worden in het bestudeerde bereik van parameters. Als we de respons van de contacthoek op het electrowettinggetal $(\eta)$ voor vlakke, cilindrische, and bolvormige geometrien vergelijken, dan kunnen we concluderen dat de geometrie van het oppervlak geen invloed heeft op het electrowettinggedrag. Onze resultaten laten zien dat de Lippmann-Young vergelijking niet alleen geldig is voor vlakke oppervlakken, maar ook - binnen het bestudeerde parameterbereik - voor bolvormige oppervlakken. Verder veronderstellen we dat het effect van oppervlaktekromming verwaarloosd kan worden in gevallen waar de dikte van de dilektrische laag veel kleiner is dan de karakteristieke dimensie van de geometrie, zoals de kromtestraal van het oppervlak $\left(\delta \ll \kappa_{2}\right)$.

Vertrouwend op de toepasbaarheid van electrowetting op niet-vlakke oppervlakken, bestuderen we de evenwichtstoestanden van twee concurrerende geometrien zowel experimenteel als analytisch, door de effectieve oppervlakte-energie van beide toestanden uit te rekenen. Onze resultaten tonen aan dat de "gedeeltelijk omsluitende" toestand energetisch voordeliger en mechanisch stabieler is, omdat de absolute effectieve oppervlakte-energie van de volledig omsluitende toestand onder alle omstandigheden groter is dan die van de "gedeeltelijk omsluitende" toestand. Het is noemenswaardig te vermelden dat de toepassing van electrowetting op bevochtigingsvraagstukken voor ingewikkelde geometrien de weg vrijmaakt om industrieel relevante vraagstukken te verkennen, zoals de mechanische stabiliteit van menisci tussen niet-vlakke substraten zoals bollen en vezels.

Hoofdstuk 6 biedt direct experimenteel bewijs voor de theoretisch voorspelde hydrodynamische instabiliteit van 1D ionische geleiding $[5,6]$. De randvoorwaarde voor het opstarten van de stroming moet expliciet onderscheiden worden van zijn multi-dimensionele equivalent (bijvoorbeeld aan een gekromd oppervlak zoals in een bolvormig ionenuitwisselingsgranulaat $[7,8,9]$ of een golvend membraanoppervlak [10], aan een membraanoppervlak met lokale verschillen in gelei- 
dbaarheid [11], of in een rij nanokanalen [12]). In deze gevallen zorgt iedere vorm van electro-osmose (EO) bij toepassing van een elektrisch veld voor een stroming, zonder dat het elektrisch veld een minimumwaarde moet overschrijden. Dus, hoewel niet-klassieke EO kwalitatief herkend kan worden aan de hogere stroomsnelheden, kan de exacte vorm van slip alleen gedentificeerd worden door de elektrisch veld-afhankelijkheid van de snelheid te bestuderen. We verwachten een lineaire afhankelijkheid in het klassieke geval van quasiequilibrium EO aan een oorspronkelijk geladen grensvlak [13] en een kwadratische afhankelijkheid voor quasiequilibrium EO met genduceerde lading aan een oorspronkelijk niet-geladen grensvlak $[14,15,16]$. Aan de andere kant wordt een derdemachts veldafhankelijkheid verwacht voor de extended charge EO, welke voor hogere voltages overgaat in een kwadratische afhankelijkheid met de tangentile variatie in de stroomdichtheid als drijfveer in plaats van het elektrische veld [5, 6]. Dit laatste stromingstype is recent beschreven voor een rij nanokanalen [12]. Het is aannemelijk dat meer beschreven vloeistofstromingen, die in het verleden aan andere mechanismes zijn toegeschreven, eigenlijk in deze categorie thuishoren $[17,18]$. 


\section{Acknowledgements}

I would like to express my sincere gratitude to everyone who helped me during my $\mathrm{PhD}$ and even after. All your support and encouragement is highly appreciated.

First of all I would like to thank my advisors Prof. Frieder Mugele and Prof. Rob Lammertink for giving me the opportunity to work on this project. Normally most of PhD students who have two advisers intend to complain, since the advisors not necessarily have the same views on the project, and in this kind of situations the student is the one who suffers. This is definitely not my case! Instead, I have got great freedom that allowed me to pursue this work. I would like to particularly thank Frieder for his innovative ideas and guidance during the project. I have learned from him a lot about scientific precision, how to plan and construct papers and give presentations. I would like to thank Rob for his great support. His advises and ability to motivate and cheer up people was invaluable during the times when I most needed it.

I would like to thank all past and present members of PCF and MTG

groups. Since the first day I felt welcomed to the Netherlands and to the group particularly. It was a pleasure to be part of this wonderful social environment.

Thanks to Adrian and Florient who thought me how to do high quality experimental research. Thanks to Chandra, Jung-Min, Amy, Christophe, Alvaro, Burak, Can, Jolet, Rielle, Hao, Sissi, Arun, Olga, Pablo, Omkar, Dhirendra, Helmut, Jane, Dieter, Huub, Mariska, Hao, Dileep (I hope did not forget anyone). I have really enjoyed working 
with you in the lab and collaborating on numerous projects. Also thanks to Daniel for nice samples.

Special thanks go to Jolet and Dileep, my best office mates of all times. It was great pleasure to share the office with you.

Another special thanks to SFI group members: Rob, Jigar, Ineke, Can, Elif and Ninke for the nice working atmosphere.

I would like to thank my ex-flatemates. Thank you Benjamin for delicious French cuisine you offered me. Thank you Burak and Annemirl for countless filling dinners with long-lasting pleasant conversations around the dinner table and much more. I enjoyed them a lot. Burak for me was not only a colleague and flat mate. He is a good friend I could always count on. Thanks to another friend of mine and my last flat mate Shavarsh. I really enjoyed your company during our trips to Zurich and Copenhagen, also long lasting PES tournaments every weekend.

Another person whom I am grateful is Can. He is a nice guy and good friend, who is always ready to lend a hand, listen, give a good advice or cheer up. I also would like to thank Jung-Min. His ability to model everything was amazing. It was a great pleasure to work with you and also to test your delicious Korean dishes.

Thanks to Jolet, Rielle, Dieter, Mariska and Huup with whom I enjoyed many "over coffee break" discussions. I liked very much our gathering around the table and playing board games until late night. Special thanks go to Helmut who is a good example of a researcher dedicated to his work. But this does not mean that he was in the lab $7 / 24$. We had excellent dinners and weekend trips organized by him. I really enjoyed the winter holydays in Austria where I tried snowboarding for the first time in my life, and now I cannot imagine me without it. 
Thanks to Secretaries of PCF, MTG and later on SFI groups Annelies, Greet and Ninke for helping me to cope with all sorts of documents and procedures I had to face during past 5 years.

Outside the work I spent some time playing my favorite game: football (or footzall). UT football club VV Drienerlo not only gave me the opportunity to play my favorite game and meet new people, but also to travel all over the country.

Last but not least, I would like to thank my family: my father Samvel, mother Susanna, and brother Edgar with his wife Ksenya for their continuous support and encouragement. I would like to thank my wife Margarita for her love, patience and support especially during my thesis writing. 


\section{About the author}

Gor Manukyan was born on 24 of Aprin 1983 in a small town Martuni, Armenia. After graduating A. Shirakaci high school in 1999 he was accepted in physics department of Yerevan State University. Four years later he received bachelor degree in laser physics. In 2004 Gor was accepted to Albert Katz international school of desert research, Ben-Gurion University of Negev, Israel. In 2004 he was awarded Master of Science degree (Cum Laude). The same year he moved from dry Israel to rainy Netherlands to start his $\mathrm{PhD}$ research in groups of Physics of Complex Fluids and Membrane Technology Group under supervision of Prof. Frieder Mugele and Prof. Rob Lammertink. The results of his work are presented in this dissertation. 